\title{
Synthesis and Applications of Asymmetric Catalysis Using Chiral Ligands Containing Quinoline Motifs
}

\author{
Vasudevan Dhayalan*a (D) \\ Rambabu Dandelab \\ K. Bavya Devic \\ Ragupathy Dhanusuramana \\ ${ }^{a}$ Department of Chemistry, National Institute of Technology \\ Puducherry, Karaikal, 609609, Union Territory Puducherry, India \\ dhaya.chem@nitpy.ac.in \\ orgdhaya@gmail.com \\ ${ }^{b}$ Department of Industrial and Engineering Chemistry, Institute of \\ Chemical Technology, Indian oil Odisha Campus, Kharagpur extension \\ Centre, Mouza, Samantpuri, Bhubaneswar-751013, Odisha, India \\ Department of Chemistry, Thassim Beevi Adbul Kader College for \\ Women, Kilakarai, Ramanathapuram, Tamilnadu, India \\ Dedicated to Professor Benjamin Lis
}

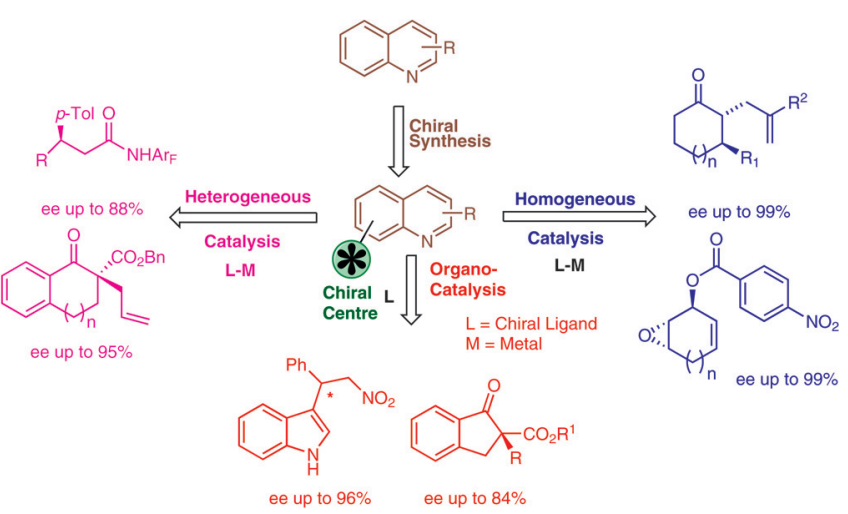

4.2 Asymmetric Heck Reactions

4.3 Asymmetric Hydrogenations

4.4 Asymmetric Hydroformylation of Styrene

4.5 Asymmetric Dialkoxylation of 2-Propenylphenols

4.6 Asymmetric Cascade Cyclizations

4.7 Asymmetric Allylic Alkylations

4.8 Asymmetric Alkylation of $\beta$-Keto Esters

4.9 Asymmetric C-H Bond Arylation Reactions

4.10 Intramolecular Aerobic Oxidative Amination of Alkenes

4.11 Asymmetric Oxidative Hydroboration of Alkenes

5 Conclusions

Abstract In the past decade, asymmetric synthesis of chiral ligands containing quinoline motifs, a family of natural products displaying a broad range of structural diversity and their metal complexes, have become the most significant methodology for the generation of enantiomerically pure compounds of biological and pharmaceutical interest. This review provides comprehensive insight on the plethora of nitrogen-based chiral ligands containing quinoline motifs and organocatalysts used in asymmetric synthesis. However, it is confined to the synthesis of quinoline-based chiral ligands and metal complexes, and their applications in asymmetric synthesis as homogeneous and heterogeneous catalysts.

1 Introduction

2 Synthesis of Chiral Ligands Containing Quinoline Motifs

2.1 Synthesis of Schiff Base Type Chiral Ligands

2.2 Synthesis of Oxazolinyl-Type Chiral Ligands

2.3 Synthesis of Chiral N,N-Type Ligands

2.4 Synthesis of Amine-Based Chiral Ligands

2.5 Synthesis of P,N-Type Chiral Ligands

2.6 Synthesis of Chiral $\mathrm{N}$-Oxide and Nitrogen Ligands

3 Homogeneous Catalytic Asymmetric Reactions

3.1 Asymmetric Carbon-Carbon Bond Formation Reactions

3.2 Asymmetric Allylic Reactions

3.3 Asymmetric Cycloadditions

3.4 Asymmetric Carbene Insertions

3.5 Asymmetric Pinacol Couplings

3.6 Asymmetric Pudovik Reactions

3.7 Asymmetric Strecker Reactions

4 Heterogeneous Catalytic Asymmetric Reactions

4.1 Asymmetric Cyclopropanation of Olefins
Key words chiral ligands, catalysis, asymmetric synthesis, nitrogen heterocycles, quinoline motifs, organometallics

\section{Introduction}

Quinoline, which is now one of the most important heterocyclic compounds, displaying a wide range of applications in pharmaceutical industries and organic synthesis, was first discovered by German chemist Friedlieb Ferdinand Runge in 1834, as a hygroscopic colorless liquid obtained by the distillation of coal tar. ${ }^{1}$ The Friedländer annulation remains one of the simplest and most straightforward methods used in organic synthesis to access highly functionalized polysubstituted quinolines. This transformation is generally accomplished through the condensation of 2aminoarylaldehydes or ketones with a ketone containing an active methylene group in the presence of acid or base. ${ }^{2-4}$ Later, numerous methods were developed for the preparation of highly substituted quinoline and its derivatives. ${ }^{5-11}$ Moreover, many quinoline derivatives exhibiting significant biological activities have been isolated from plants or systematically designed and synthesized. ${ }^{12,13}$ 
Quinolines and their derivatives have been labeled as 'privileged scaffolds' owing to their prevalent existence in natural and synthetic molecules that exhibit notable appli- cations in pharmacological, agrochemical, and electronic industries (Figure 1). ${ }^{14-24}$

\section{Biographical Sketches \\ Dr. Vasudevan Dhayalan ob- tained his MSc in organic chem- istry (2005) and his $\mathrm{PhD}$ in organic chemistry (2011) at the University of Madras, Chennai, India. Then he received post- doctoral research experience \\ Prof. Paul Knochel, Ludwig-Max- imilians-University, Munich, Germany, and Prof. Anat Milo, Ben-Gurion University of the Negev, Beer Sheva, Israel. He is a recipient of a PBC Outstand- ing Postdoctoral Research Fel- \\ ship from the Science and Engi- neering Research Board (SERB), India. Currently, he is working as a Ramanujan Fellow (Assistant Professor) at the National Insti- tute of Technology Puducherry, Karaikal, India.}

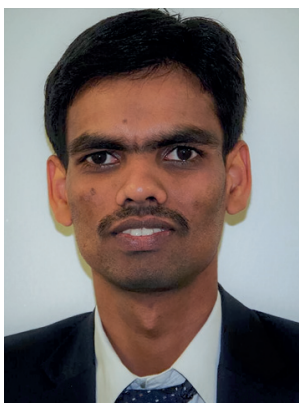
with Prof. Masahiko Hayashi, Kobe University, Kobe, Japan,
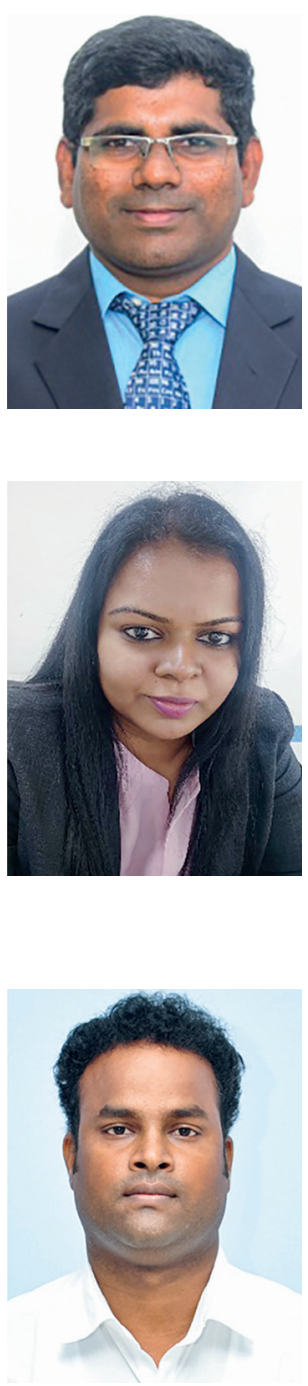
8. After that, she obtained her $\mathrm{PhD}$ in the Department of Chemistry, Anna University

\section{Dr. Ragupathy Dhanusura-} man received an MSc degree in chemistry from Bharathiar University, Coimbatore, India (2005). He completed his PhD in the Department of Chemistry, lowship. Recently, he was awarded a Ramanujan Fellow-
Fellow. In 2018, he became an Assistant Professor of Chemistry at the Institute of Chemical Technology, Indian Oil Odisha Campus, Bhubaneswar. His research interests lie at the interface of chemistry and biology with particular focus on structure-based drug design, bacterial signaling and polymorphism in pharmaceutical solids.

gal Roy's group as a post-doctoral researcher in the Department of Metallurgical and Materials Engineering at the Indian Institute of Technology Kharagpur, India, in 2015. Her research interests are primarily focused on the development of new degradable materials that support bone regeneration. search collaboratively with the Bhabha Atomic Research Centre (BARC) Mumbai. Dr. Bavya has received eight best paper awards in both national and international conferences. She was awarded a National Doctoral Fellowship (NDF) by the All India Council of Technical Education, New Delhi, India. Later, she joined Professor Man-

Kyungpook National University, South Korea (2010). Currently, he is working as an Assistant Professor in the Department of Chemistry, National Institute of Technology Puducherry, Karai- kal, India. His research interests include organic polymers and the development of new nanomaterials for energy and electro-analytical applications. 


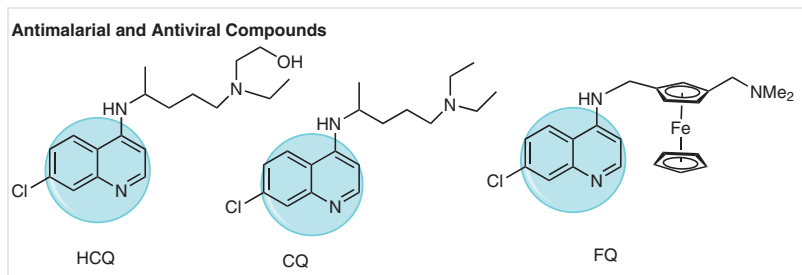

Bioactive Compounds
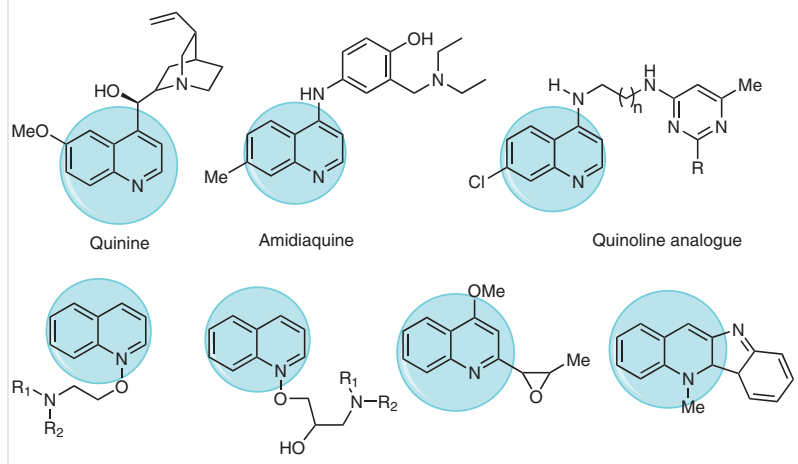

Anticonvulsant and antihypertensive drugs

Leishmaniasis agent

Cryptolepine drug

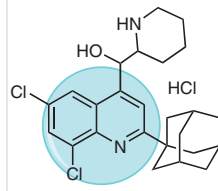

NSC-305787

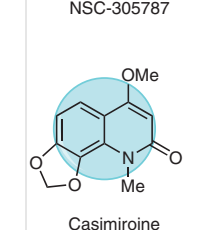

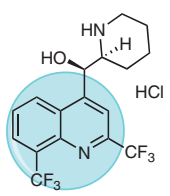

Lariam

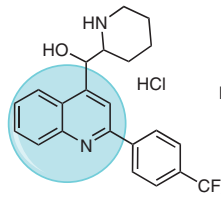

Bacterial biofilms

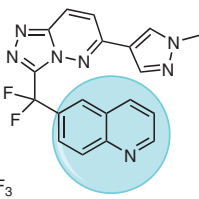

JNJ38877605
Casimiroine

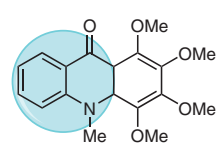

Melicopicine

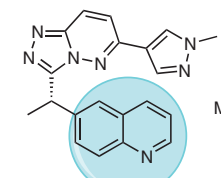

PF-04254644

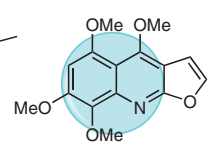

Acronycidine

Figure 1 Examples of biologically active quinolines

Furthermore, quinoline analogues display antimalarial, ${ }^{25-27}$ anti-inflammatory, antitumor, antibacterial, antivi$\mathrm{ral}^{28}{ }^{28}$ anticonvulsant, ${ }^{29}$ and cardiovascular ${ }^{30}$ activities. ${ }^{31} \mathrm{Nu}-$ merous studies on enantioselective catalysis have focused on the development of novel chiral ligands for use in organo- and transition-metal-catalyzed asymmetric reactions. $^{32-41}$ Recently, Benjamin List and David MacMillan have clearly demonstrated the significance of organocatalyst in asymmetric synthesis. ${ }^{41}$ Given the broad structural diversity of bicyclic nitrogen heterocycles, these recent Nobel Laureates have demonstrated the ability of these compounds to catalyze a range of chemical transformations. The ease with which many such quinoline (benzo[b]pyridine) ring systems can be synthetically modified within chiral scaffolds ${ }^{42-67}$ can lead to the discovery of new enantioselective processes for the synthesis of highly challenging chiral products with interesting applications in biology.

\section{Synthesis of Chiral Ligands Containing Quinoline Motifs}

\subsection{Synthesis of Schiff Base Type Chiral Ligands}

In 2008, Hayashi and co-workers reported the preparation of the N,N,P-ligands. The N,N,P-tridentate Schiff base ligands $\mathbf{6 a}, \mathbf{b}$ were prepared from chiral amino alcohols $\mathbf{1}$ in five steps with high yields (Scheme 1). The synthetic pathway started from chiral amino alcohols $\mathbf{1}, \mathrm{NH}$ and $\mathrm{OH}-$ tosylation of which, followed by treatment with potassium hydroxide $(\mathrm{KOH})$ gave the anticipated substituted aziridines 3a,b. The obtained amine-protected aziridines $\mathbf{3}$ were treated with $\mathrm{KPPh}_{2}$ to afford the corresponding $\mathrm{N}$-tosylated amino phosphines 4. Simple condensation of 2-quinolinecarboxaldehyde with these $\mathrm{N}-\mathrm{H}$ free amino phosphines $\mathbf{5}$ gave the expected N,N,P-tridentate chiral Schiff bases $\mathbf{6 a}, \mathbf{b}$ in good yields. These N,N,P-tridentate Schiff base ligands were used quinoline-based asymmetric catalysts in organic synthesis, such as 1,4 -addition of $R_{2} Z n$ to $\alpha, \beta$-unsaturated ketones.

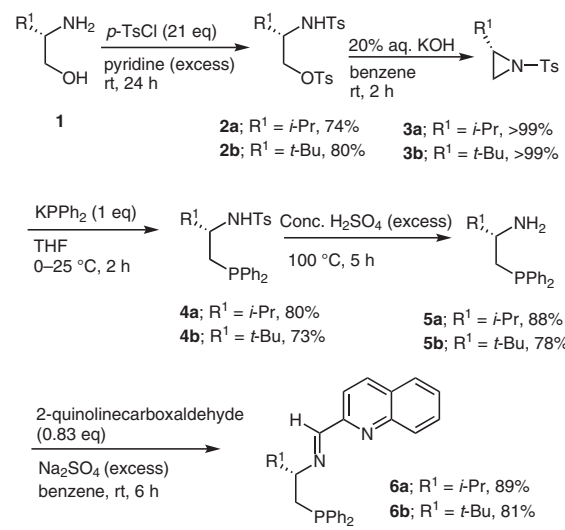

Scheme 1 Synthesis of chiral N,N,P-tridentate Schiff base ligands ${ }^{68}$

In the same year, Hayashi and co-workers reported the preparation of a library of chiral Schiff base ligands 11a-f (Scheme 2). Chiral imines were readily prepared by condensation of aldehydes $\mathbf{7}$ or ketones $\mathbf{9}$ with chiral amines. The keto-imine chiral Schiff bases, 11a-f were prepared by two different methods. In method 1, the addition of a Grignard reagent ${ }^{69 a}$ to 2 -quinolylaldehyde 7 produced the desired alcohol $\mathbf{8}$ in good yields; the obtained secondary alcohol was then effectively converted into the corresponding ketones $\mathbf{9}$ via a radical oxidation process. In method 2 , treatment of 2 quinoline cyanide $\mathbf{1 0}$ with a Grignard reagent furnished the required ketones $\mathbf{9}$. Finally, condensation of ketones 9 with chiral amino alcohols in the presence of $\mathrm{TiCl}_{4}$ and $\mathrm{Et}_{3} \mathrm{~N}$ gave the corresponding chiral ligands 11a-f (Scheme 2). These $\mathrm{N}, \mathrm{N}$-bidentate Schiff base ligands were applied to the allylic oxidation of olefins. 


\section{.} (up to $85 \%$
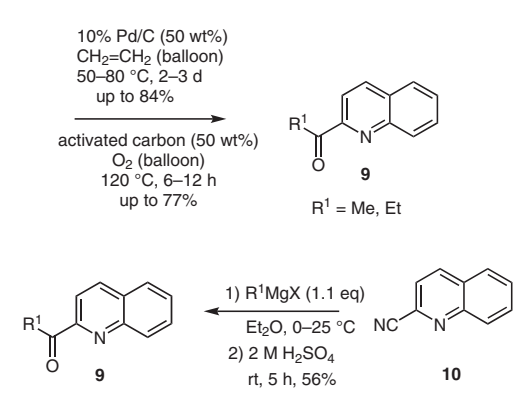

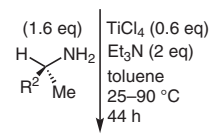

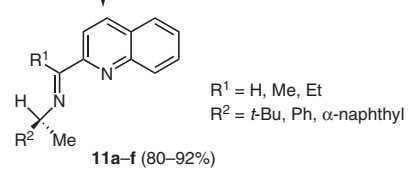

Scheme 2 Synthesis of chiral keto-imine N,N-bidentate Schiff base ligands ${ }^{69 b, c}$

In 2004, Suga and co-workers investigated the synthesis of diamine type chiral Schiff base ligands 14a-f. Aldimine type chiral Schiff bases were synthesized by simple condensation of substituted 2/8-quinolylaldehyde $\mathbf{1 3 a}$,b with chiral 1,1'-binaphthyldiamine $\mathbf{1 2}$ in benzene under reflux (Scheme 3). These binaphthyldiimine Schiff base ligands were found to be widely applicable to various 1,3-dipolar cycloaddition reactions and Diels-Alder reactions.

Eddine and co-workers demonstrated the preparation of iminium salt $\mathbf{1 7}$ via halogen-metal exchange reaction of (R)-2-(sec-butoxy)bromonaphthalene (15) with $n$-BuLi at low temperature to furnish the aryl lithium species $\mathbf{1 6}$, which, upon subsequent nucleophilic addition to 8-cyanoquinoline followed by quenching with methyl iodide (Scheme 4), furnished the corresponding chiral $N$-methyl1-(8-quinolinyl)-1-(2-(R)-sec-butoxynaphthyl)-methylenimime ligand 17 (Scheme 4). These keto-imine type chiral Schiff base ligands were examined in effective phase-transfer catalyzed asymmetric alkylation reactions.

\subsection{Synthesis of Oxazolinyl-Type Chiral Ligands}

In 1999, Chelucci and co-workers designed a simple synthesis of chiral oxazolinylquinoline type ligands $\mathbf{2 1}$ and 22. Oxidation of quinoline $\mathbf{1 8}$ with 3 -chloroperbenzoic acid ( $m$-CPBA) in DCM for $2 \mathrm{~h}$ and then treatment of the obtained $\mathrm{N}$-oxide with 2.0 equivalents of $\mathrm{KCN}$ and $\mathrm{PhCOCl}$ in $\mathrm{CH}_{3} \mathrm{CN} / \mathrm{MeOH}$ at room temperature for $24 \mathrm{~h}$, produced the corresponding compound 19. Subsequent treatment with 2-cyanoquinoline 19 and chlorobenzene under reflux with
Review

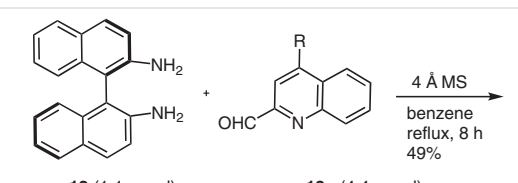

$12(1.1 \mathrm{mmol})$

13a $(4.4 \mathrm{mmol})$<smiles></smiles>

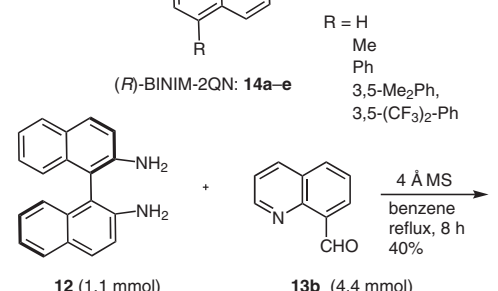

$12(1.1 \mathrm{mmol})$

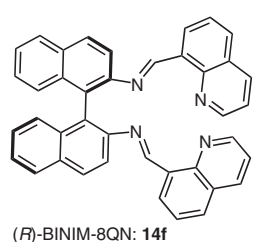

(R)-BINIM-8QN: $14 \mathrm{f}$

Scheme 3 Synthesis of binaphthyldiimine chiral Schiff base ligands ${ }^{70}$

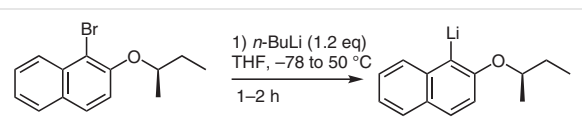

15

16
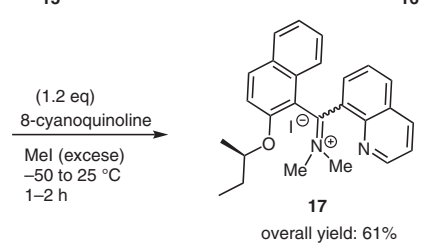

Scheme 4 Synthesis of a keto-imine chiral Schiff base ligand ${ }^{71}$

the addition of a suitable chiral amino alcohol 20a in the presence of $\mathrm{ZnCl}_{2}$ produced the corresponding oxazolinylquinoline type chiral ligands $\mathbf{2 1}$ and $\mathbf{2 2}$ in yields of 10-99\% (Scheme 5). In a similar manner, chiral quinoline ligands 24a-c were synthesized from 2-cyanomethylquinoline 23 and the corresponding amino alcohol 20a, mediated by $\mathrm{ZnCl}_{2}$ under reflux conditions (Scheme 5 ).

Chelucci (2000) and co-workers expanded the library of quinoline ligands, by synthesizing chiral oxazolines 28a-e, employing an amide-mesylate-oxazoline reaction sequence (Scheme 6). Thus, 8-quinolyl carboxylic ester 25 was heated in toluene under reflux with chiral amino alcohol 26a in the presence of potassium cyanide to afford the corresponding amide derivates $\mathbf{2 7}$ in quantitative yields. Finally, the quinolyloxazolines $\mathbf{2 8 a}$-e were obtained by the reaction of amino alcohol 27 with methane sulfonyl chlo- 


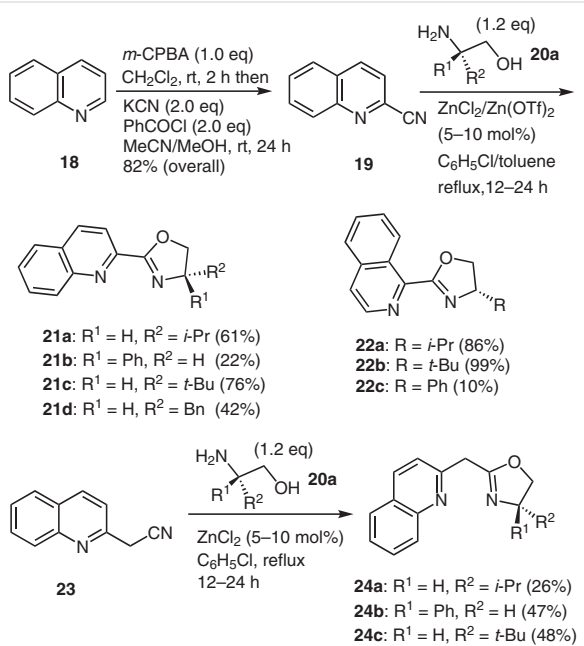

Scheme 5 Synthesis of N,N-chiral 2-quinolyloxazoline ligands ${ }^{72}$

ride $\left(\mathrm{MeSO}_{2} \mathrm{Cl}\right)$ and $\mathrm{Et}_{3} \mathrm{~N}$ in DCM solvent. The obtained chiral ligands 28a-e were air-sensitive and, upon standing at $25{ }^{\circ} \mathrm{C}$, underwent a ring opening reaction to furnish the amide by-products. The chiral ligand 28d was treated with metal salts such as $\mathrm{Cu}(\mathrm{OTf})_{2}$ or $\mathrm{PdCl}_{2}$ to give the corresponding oxazoline transition-metal complexes 29. These chiral quinolyloxazoline ligands were studied in FriedelCrafts alkylation, cyclopropanation of olefins, cascade intramolecular cyclization reactions, dialkoxylation of 2-alkenes, intramolecular aerobic oxidative amination, and allylic alkylation.

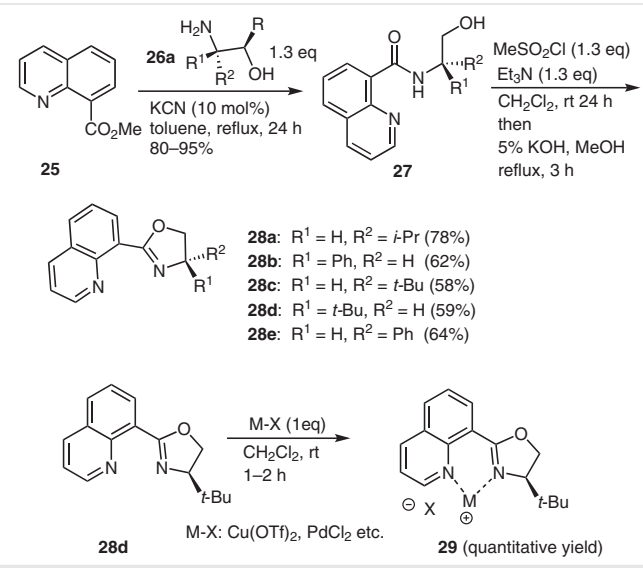

Scheme 6 Synthesis of N,N-chiral 8-quinolyloxazoline ligands ${ }^{72 b, 73}$

Muller and co-workers (2000) reported an innovative synthesis of oxazolinyl ligands $\mathbf{3 1}$ containing a hydroxyl group and silyl group 32 (Scheme 7). The central chiral ligand was prepared by one-pot cyclization of 8-cyanoquinoline and chiral amino benzyl alcohol $\mathbf{2 6 b}$ at $110{ }^{\circ} \mathrm{C}$ in the presence of ethylene glycol. The obtained oxazolinyl-OH li- gand 31 was protected using TBDPSCl in the presence of imidazole at room temperature for $24 \mathrm{~h}$. Likewise, the benzyl protected oxazolinyl ligand $\mathbf{3 3}$ was prepared.

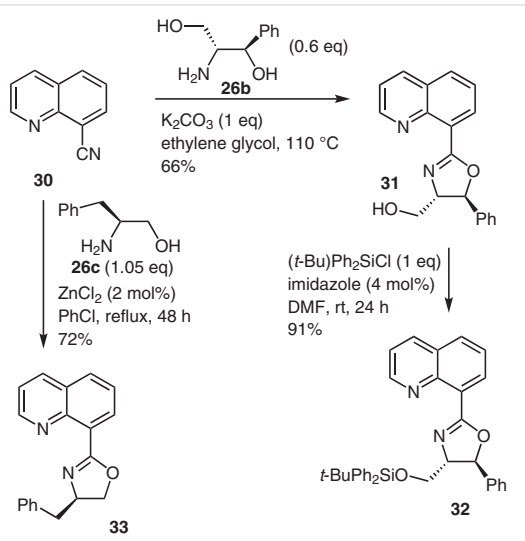

Scheme 7 Synthesis of silyl protected chiral oxazolinyl ligands ${ }^{74}$

Ahn and co-workers (1999) designed and synthesized 8diarylphosphino-2-oxazolinylquinoline type chiral ligands 38a-c starting from 35. 2-Cyano-8-hydroxyquinoline precursor 35, synthesized from 8-hydroxyquinoline $\mathbf{3 4}$ according to the literature, ${ }^{75}$ was used for the preparation of the target chiral ligands. The authors reported that $\mathrm{ZnCl}_{2}$-catalyzed oxazolinyl ring formation furnished better yields when the quinoline-alcohol group was converted into the corresponding aryl triflate $\mathbf{3 6}$; otherwise, in the presence of the quinoline free $\mathrm{OH}$ group, oxazolinyl ligand derivatives 37 were obtained in lower yields. The condensation of L-valinol 20b and aryl cyanide $\mathbf{3 6}$ in the presence of $\mathrm{ZnCl}_{2}(10$ $\mathrm{mol} \%$ ) with $\mathrm{PhCl}$ as a solvent, afforded the resulting oxazolines 37a-c in good yields. Introduction of the diphenylphosphino group $\left(\mathrm{PPh}_{2}\right)$ was accomplished by Ni-catalyzed $\mathrm{C}-\mathrm{P}$ coupling. Thus, reaction of the oxazoline derivatives 37 with diphenylphosphine in the presence of $\mathrm{NiCl}_{2}$ (dppe) (10 mol\%) and 1,4-diazabicyclo[2.2.2]octane (DABCO, 2 equiv) in DMF at $80^{\circ} \mathrm{C}$ afforded the subsequent N,N,P-ligands 38ac in moderate isolated yields. When the coupling reaction was carried out at $100{ }^{\circ} \mathrm{C}$ or above, as previously reported, the reaction yield was diminished (Scheme 8).

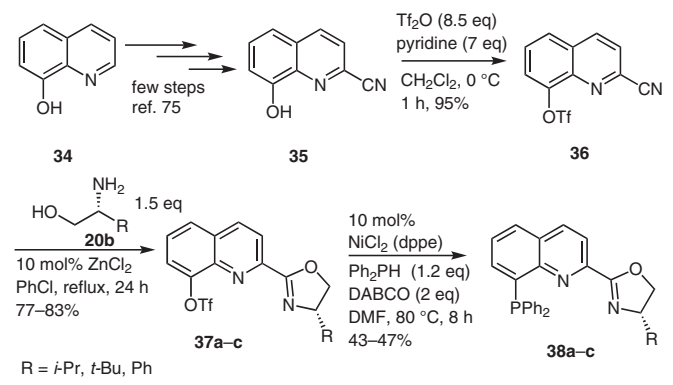

Scheme 8 Synthesis of N,N,P-chiral 2-oxazolinylquinoline ligands ${ }^{75}$ 


\subsection{Synthesis of Chiral N,N-Type Ligands}

Bolm and co-workers prepared a wide range of quinoline-based $C_{1}$-symmetric chiral monosulfoximine derivatives 41a-l, in which the second donor nitrogen atom is in a quinolinyl aromatic ring, by $\mathrm{Pd}(\mathrm{OAc})_{2}$-catalyzed $\mathrm{N}$-arylation of optically pure sulfoximines $\mathbf{3 9}$ with the corresponding 8-bromoquinoline derivatives $\mathbf{4 0}$. The chiral sulfoximine substrate scope is summarized in Scheme 9.

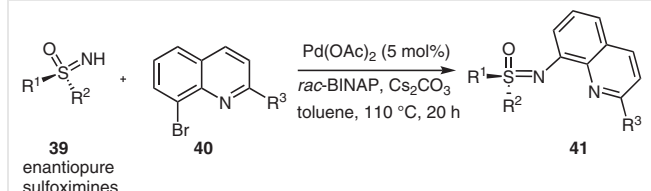

41g; $R^{1}=\mathrm{Me}, \mathrm{R}^{2}=3,5$-di-t-Bu-Ph, $\mathrm{R}^{3}=\mathrm{H}(81 \%)$ $\begin{array}{ll}41 \mathrm{a} ; \mathrm{R}^{1}=\mathrm{Me}, \mathrm{R}^{2}=\mathrm{Ph}, \mathrm{R}^{3}=\mathrm{H}(90 \%) & 41 \mathrm{~g} ; \mathrm{R}^{1}=\mathrm{Me}, \mathrm{R}^{2}=3,5-\mathrm{di}-\mathrm{t}-\mathrm{Bu}-\mathrm{Ph}, \mathrm{R}^{3}=\mathrm{H}(81 \%) \\ 41 \mathrm{~b} ; \mathrm{R}^{1}=\mathrm{Me}, \mathrm{R}^{2}=\mathrm{Ph}, \mathrm{R}^{3}=n-\mathrm{Bu}(75 \%) & 41 \mathrm{~h} ; \mathrm{R}^{1}=\mathrm{Me}, \mathrm{R}^{2}=2-\mathrm{MeO}-\mathrm{Ph}, \mathrm{R}^{3}=\mathrm{H}(87 \%) \\ 41 \mathrm{c} ; \mathrm{R}^{1}=\mathrm{Me}, \mathrm{R}^{2}=\mathrm{Ph}, \mathrm{R}^{3}=4 \mathrm{Br} \text {-acridine }(68 \%) & 14 \mathrm{i} ; \mathrm{R}^{1}=n \text {-pentyl, } \mathrm{R}^{2}=2-\mathrm{MeO}-\mathrm{Ph}, \mathrm{R}^{3}=\mathrm{H}(85 \%)\end{array}$ $\begin{array}{ll}\text { 41c; } \mathrm{R}^{1}=\mathrm{Me}, \mathrm{R}^{2}=\mathrm{Ph}, \mathrm{R}^{3}=4 \mathrm{Br} \text {-acridine }(68 \%) & 14 \mathrm{i} ; \mathrm{R}^{1}=n \text {-pentyl, } \mathrm{R}^{2}=2-\mathrm{MeO}-\mathrm{Ph}, \mathrm{R}^{3}=\mathrm{H}(85 \%) \\ 41 \mathrm{~d} ; \mathrm{R}^{1}=i-\mathrm{Pr}, \mathrm{R}^{2}=\mathrm{Ph}, \mathrm{R}^{3}=\mathrm{H}(75 \%) & 41 \mathrm{j} ; \mathrm{R}^{1}=\text { phenethyl, } \mathrm{R}^{2}=2-\mathrm{MeO}-\mathrm{Ph}, \mathrm{R}^{3}=\mathrm{H}(73 \%)\end{array}$ $\begin{array}{ll}41 \mathrm{~d} ; \mathrm{R}^{1}=i-\mathrm{Pr}, \mathrm{R}^{2}=\mathrm{Ph}, \mathrm{R}^{3}=\mathrm{H}(75 \%) & 41 \mathrm{j} ; \mathrm{R}^{1}=\text { phenethyl, } \mathrm{R}^{2}=2-\mathrm{MeO}-\mathrm{Ph}, \mathrm{R}^{3}=\mathrm{H}(73 \%) \\ 41 \mathrm{e} ; \mathrm{R}^{1}=t-\mathrm{Bu}, \mathrm{R}^{2}=\mathrm{Ph}, \mathrm{R}^{3}=\mathrm{H}(72 \%) & 41 \mathrm{k} ; \mathrm{R}^{1}=t-\mathrm{Bu}, \mathrm{R}^{2}=2-\mathrm{MeO}-\mathrm{Ph}, \mathrm{R}^{3}=\mathrm{H}(55 \%)\end{array}$ 41f; $R^{1}=M e, R^{2}=$ biphenyl, $R^{3}=H(84 \%) \quad 411 ; R^{1}=t-B u, R^{2}=2-M e O-N a p h, R^{3}=H(81 \%)$

Scheme 9 Synthesis of monosulfoximine chiral ligands ${ }^{76}$

Several N,N-bidentate type chiral quinoline derivatives have been prepared from the corresponding ketones, as reported by Chelucci and co-workers in 2000. Chiral ligands 45a-c were prepared by the reaction of quinoline ketone 42 with vinyl ketone $\mathbf{4 3}$ (Scheme 10) to produce the desired chiral quinoline intermediate $\mathbf{4 4}$, which was subsequently deprotonated with LDA at $-78{ }^{\circ} \mathrm{C}$ and then treated with alkyl or benzyl iodide to give the corresponding alkylated ligands 45a-c.

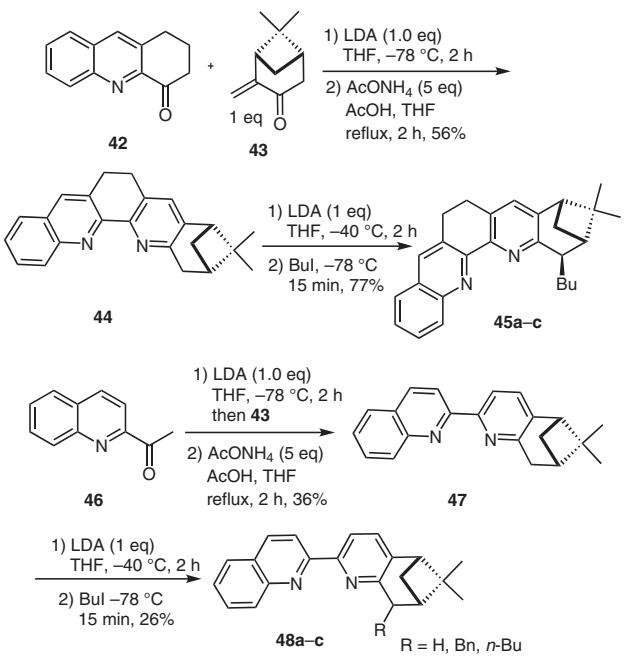

Scheme 10 Synthesis of chiral N,N-bidentate quinoline ligands ${ }^{77}$

Quinoline analogues $\mathbf{4 8 a - c}$ were successfully synthesized from methyl ketones $\mathbf{4 6}$ under similar reaction conditions (Scheme 10) using LDA and alkyl halides.
A new class of chiral ligands containing the quinoline moiety 51a-d was developed by Yamamoto and co-workers in 2004. The coupling reaction of bis-aryl iodo compound $\mathbf{4 9}$ with quinoline derivatives $\mathbf{5 0}$ in the presence of LDA and $\mathrm{BBr}_{3}$ furnished the required bis quinoline compounds 51ad (Scheme 11). Chiral ligands 51a-d were then treated with $\mathrm{Et}_{2} \mathrm{AlCl}$ or $\mathrm{CrCl}_{2}$ to give the corresponding tethered bis(8quinolinato) (TBOx) aluminum complexes 52a-d in good yields. These $N, N$-quinoline ligands were applied in pinacol couplings, the Pudovik reaction, hydrogenation of ketones and allylic alkylations.
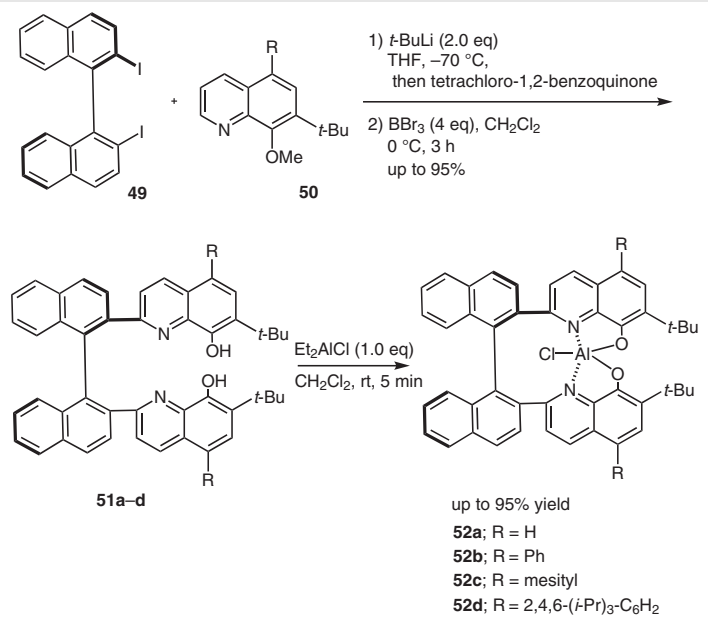

Scheme 11 Synthesis of N,N,O-chiral bis-quinoline ligands ${ }^{78}$

\subsection{Synthesis of Amine-Based Chiral Ligands}

In 2007, Romanelli and co-workers reported a series of quinoline ligands $\mathbf{5 7}$ prepared by the alkylation of alcohol 53 in the presence of $\mathrm{TsCl}$ (1.2 equiv) and pyridine at room temperature. Subsequent alkylation of 6-hydroxyquinoline 55 with Ts-ester 54 in the presence $\mathrm{NaH}$ (2 equiv) in DMF at $80^{\circ}$ for $4 \mathrm{~h}$ was followed by reduction with LAH and then MeI was added to furnish the required chiral amine salt $(R)-57$ in high yield (Scheme 12).

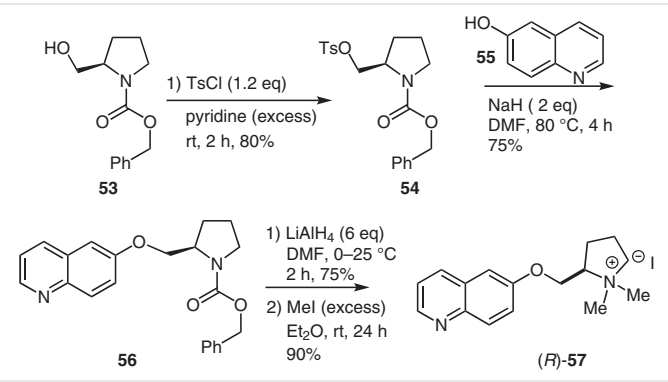

Scheme 12 Synthesis of chiral quinoline with amine salt $\mathbf{5 7 ^ { 7 9 }}$ 
Kwong et al. introduced a novel synthesis of bisamide ligand-containing quinolines, whose asymmetric synthesis started from condensation of cyclohexyl diamine $\mathbf{5 9}$ with heterocyclic aldehydes $\mathbf{5 8}$ and $\mathbf{6 1}$ to give amide-based unsymmetrical ligands $\mathbf{6 2}$ and symmetrical ligands $\mathbf{6 0}$ in good yields (Scheme 13).

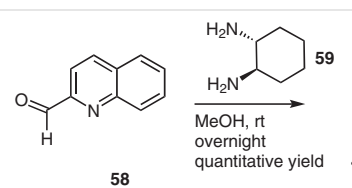

58

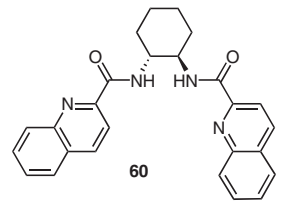

(2)
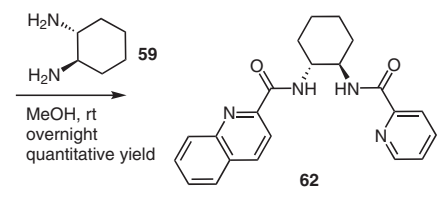

Scheme 13 Synthesis of amide-type chiral ligands ${ }^{80}$

Judeh and co-workers described a series of quinoline ligand derivatives $\mathbf{6 8} \mathbf{a}-\mathbf{m}$, whose synthesis starts from simple condensation of phenylethylamine $\mathbf{6 3}$ with diethyl oxalate in ethanol to give compound $\mathbf{6 4}$ in high yield (Scheme 14). Then, rac-65 was synthesized under double BischlerNapieralski conditions. Bis-amide $\mathbf{6 4}$ was then reacted with polyphosphoric acid (PPA) at $190{ }^{\circ} \mathrm{C}$ for $12 \mathrm{~h}$ to furnished the target compound rac-65 in 86\% yield. Reaction of compound 65 with a stoichiometric amount of enantiopure $(S)$ (-)- $\alpha$-methylbenzyl isocyanate furnished the diastereomeric urea analogues 66a and 66a' in excellent yield. When a solution of 66a or 66a' was treated with $n$-BuONa in warm $n$-BuOH (Scheme 14), the cleaved products (+)-67a and (-)67a', were obtained in up to $61 \%$ yield and $99 \%$ ee. Fortunately, one of the products could be recrystallized from ethanol and gave a very high enantiomeric excess $>99 \%$.

Various alkyl groups were introduced by reaction of (+)67 with alkyl halides in the presence of $\mathrm{K}_{2} \mathrm{CO}_{3}$ with $\mathrm{CH}_{3} \mathrm{CN}$ as a solvent at $50{ }^{\circ} \mathrm{C}$. Likewise, compound (+)-67 reacted with 1 equivalent of isocyanates and thioisocyanates in DCM at room temperature to give the target products $68 \mathbf{a}-$ $\mathbf{m}$ in excellent yields (Scheme 14).

Yus and co-workers studied a practical method for the preparation of camphor sulfonamide-based quinoline ligands 71a,b. Their synthesis started from cyclohexyldiamine $\mathbf{5 9}$ by reaction with arylsulfonyl chloride in two steps, followed by treatment with camphor sulfonyl methyl chloride 70. Friedlander annulation in the presence of ruthenium chloride as a catalyst then furnished the expected camphor sulfonamide-based quinoline ligands 71a,b in moderate yields (Scheme 15).

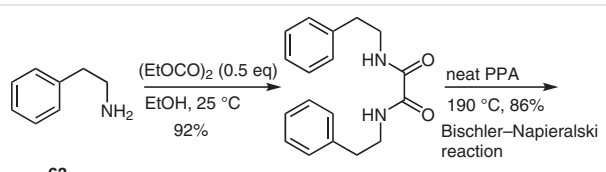

64

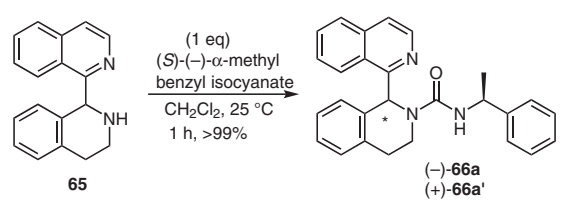

65 $(-)-66 a$
$(+)-66 a^{\prime}$

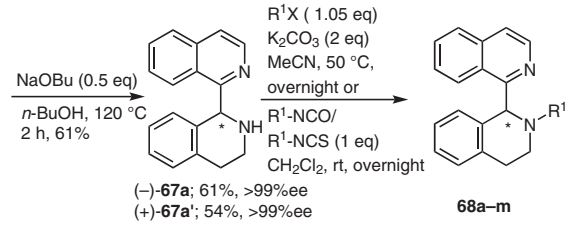

68a; $R^{1}=\mathrm{H}(61 \%)$ 68b; $\mathrm{R}^{1}=\mathrm{Me}(76 \%) \quad 68 \mathrm{i} ; \mathrm{R}^{1}=\mathrm{CSNHC}_{6} \mathrm{H}_{3}-3,5-\mathrm{CF}_{3}(87 \%)$ 68c; $\mathrm{R}^{1}=\mathrm{Et}(92 \%) \quad 68 \mathrm{j} ; \mathrm{R}^{1}=\mathrm{CONHC}_{6} \mathrm{H}_{4}-2-\mathrm{Cl}(99 \%)$ 68: $\mathrm{R}^{1}=\mathrm{CH}_{2}-\mathrm{C}_{2}-2-\mathrm{OH}-5-\mathrm{NO}_{2}(91 \%) 681 ; \mathrm{R}^{1}=\mathrm{CONH}\left(\mathrm{CH}_{2}\right) \mathrm{Me}^{2}(96 \%)$ 68f; $\mathrm{R}^{1}=\mathrm{SO}_{2}-\mathrm{C}_{6} \mathrm{H}_{4}-p-\mathrm{Me}(99 \%) \quad 68 \mathrm{~m} ; \mathrm{R}^{1}=\mathrm{CSNHEt}(90 \%)$ 68g; $R^{1}=A C(89 \%)$

Scheme 14 Synthesis of chiral isoquinoline amine ligands ${ }^{81}$
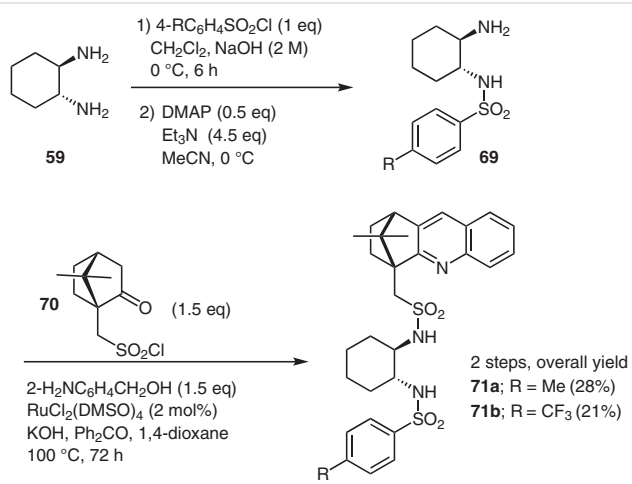

Scheme 15 Synthesis of camphor sulfonamide-based quinoline ligands 82

Felluga et al. efficiently synthesized the enantiopure amine-based ligands 75. Baker's yeast mediated reduction of methyl ketone $\mathbf{7 2}$ afforded alcohol (S)-73. However, the required chiral alcohol $(S)$-73 could also be obtained by a kinetic resolution approach. Thus, azide precursors $(R)-74$ were obtained in good yield from the benzyl alcohol in the presence of DPPA/DBU and reduction with triphenylphosphine $\left(\mathrm{Ph}_{3} \mathrm{P}\right)$ led to the desired amine ligands 75 with high enantioselectivity (Scheme 16).

Osmium metal complexes $\mathbf{7 7}$ and $\mathbf{7 8}$ were prepared by treatment of $\left[\mathrm{OsCl}_{2}\left(\mathrm{PPh}_{3}\right)_{3}\right]$ with $(S, R)$-Josiphos (1.2 equiv) in mesitylene at $110{ }^{\circ} \mathrm{C}$ for $2 \mathrm{~h}$ to give an uncharacterized mixture of products, which was then reacted with 2 -aminomethylbenzo[ $h]$ quinoline $\mathbf{7 5}$ (1.4 equiv) in the presence of triethylamine $\left(\mathrm{Et}_{3} \mathrm{~N}\right)$ at $140{ }^{\circ} \mathrm{C}$ for $24 \mathrm{~h}$ to furnish the cor- 

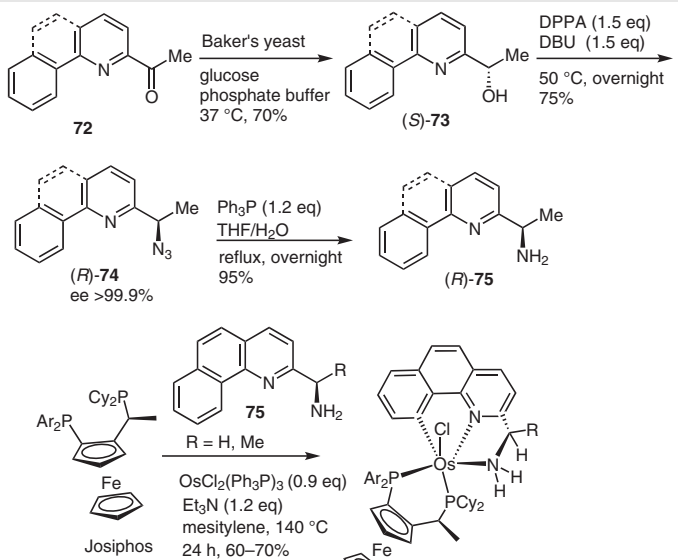

76

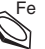

77-78

\begin{tabular}{c|ccc} 
& diphosphane & $\mathrm{Ar}$ & $\mathrm{R}$ \\
\hline 77 & $(S, R)$-Josiphos & $\mathrm{Ph}$ & $\mathrm{H}$ \\
78 & $(S, R)$-Josiphos & 4-OMe-3,5-- $\mathrm{Me}_{2} \mathrm{C}_{6} \mathrm{H}_{2}$ & $\mathrm{Me}$
\end{tabular}

Scheme 16 Synthesis of chiral benzo[ $h]$ quinoline ligands and their osmium complexes ${ }^{83}$

responding coordination metal complexes $\mathbf{7 7}$ and $\mathbf{7 8}$ in good yields (Scheme 16). These amine-based ligands were studied in catalytic applications such as 1,2-addition of organozinc reagents to substituted aldehydes, 1,4-addition of Grignard reagents ( $\left.\mathrm{R}^{1} \mathrm{MgX}\right)$ to cyclic enones, allylic alkylations, and $\mathrm{C}-\mathrm{H}$ bond arylation reactions

\subsection{Synthesis of P,N-Type Chiral Ligands}

The efficient synthesis of QUIPHOS type chiral ligands $\mathbf{8 1 a}-\mathbf{h}$ by the reaction of phosphane $\mathbf{7 9}$ and pyrrolidine $\mathbf{8 0}$ followed by addition of hydroxyquinoline $\mathbf{3 4}$ (method 1 , Scheme 17) afforded the desired ligands $\mathbf{8 1 a - h}$ in moderate to good yields, as reported by Buono and co-workers. Applying a similar reaction protocol led to a wide range of $\mathrm{P}, \mathrm{N}-$ quinoline-phosphine ligand derivatives 83a-h; selected examples are shown in method 2, Scheme 17.

Quinoline-based chiral Pd complex 87 was effectively prepared via halogen-metal ( $\mathrm{Li}-\mathrm{Br}$ ) exchange of heterocyclic bromo compound 84 and $s$-BuLi, followed by quenching with $\mathrm{PCl}\left(\mathrm{NMe}_{2}\right)_{2}$ to give quinoline-phosphine ligand 85 . Reaction of $\mathrm{P}, \mathrm{N}$-ligand $\mathbf{8 5}$ with chiral amine $\mathbf{8 0}$ produced the corresponding chiral ligand $\mathbf{8 6}$ and this was treated with $\left[\mathrm{PdCl}_{2}\left(\mathrm{CH}_{3} \mathrm{CN}\right)_{2}\right]$ in DCM to produce the desired $N, N, P-\mathrm{Pd}$ complex 87 in excellent yield (Scheme 18).

The phosphonito, nitrogen ligand $(R)-\mathbf{9 0}$ has been synthesized in a one-pot, two-step process (Scheme 19). transMetalation of 8-bromoquinoline 84 with $n$-butyllithium ( $n$-BuLi) and subsequent treatment with $\mathrm{PCl}\left(\mathrm{NEt}_{2}\right)_{2}$ to form

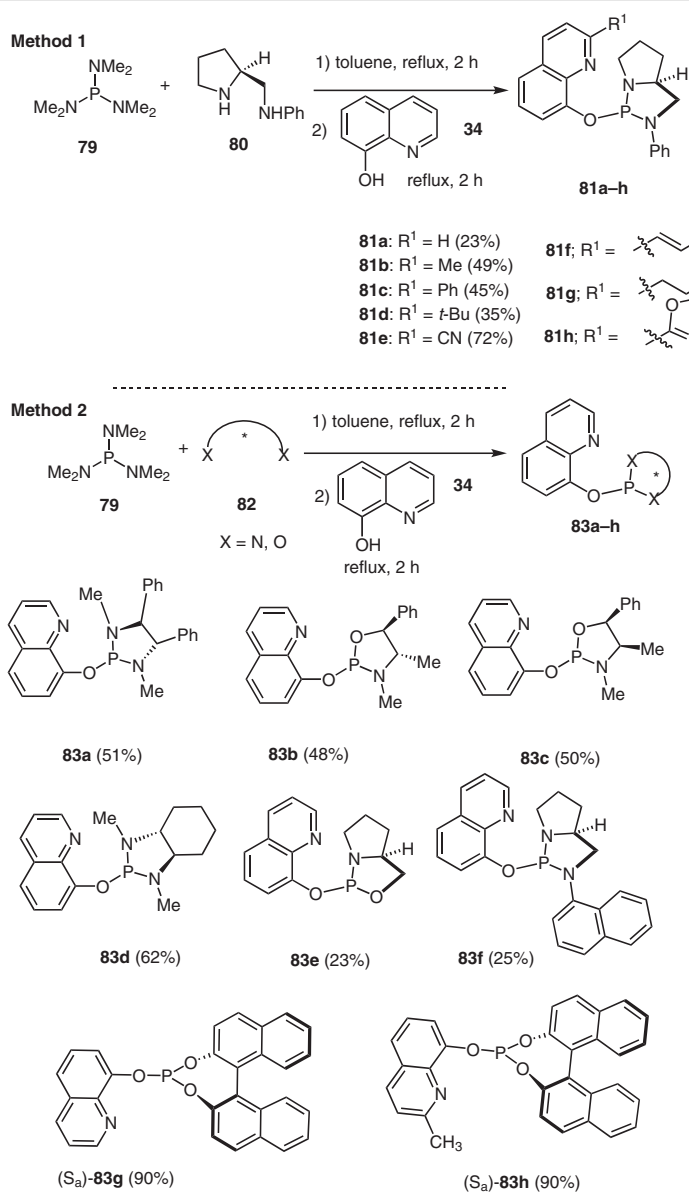

Scheme 17 Synthesis of chiral N,P,O-quinoline-phosphine ligands ${ }^{84}$
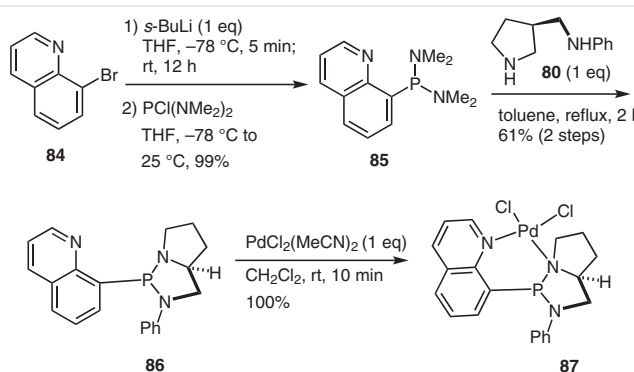

Scheme 18 Synthesis of N,N,P-chiral quinoline-phosphine ligand $\mathbf{8 6}$ and its Pd complex ${ }^{85}$

phosphine compound $\mathbf{8 8}$, followed by the reaction with $(R)$-binaphthol 89 in toluene at reflux, furnished P,N-ligand $(R)-90$ in good yield. This ligand was reacted with Pd, Pt and Rh complexes to furnish the desired metal complexes 91-93 in good yields. 


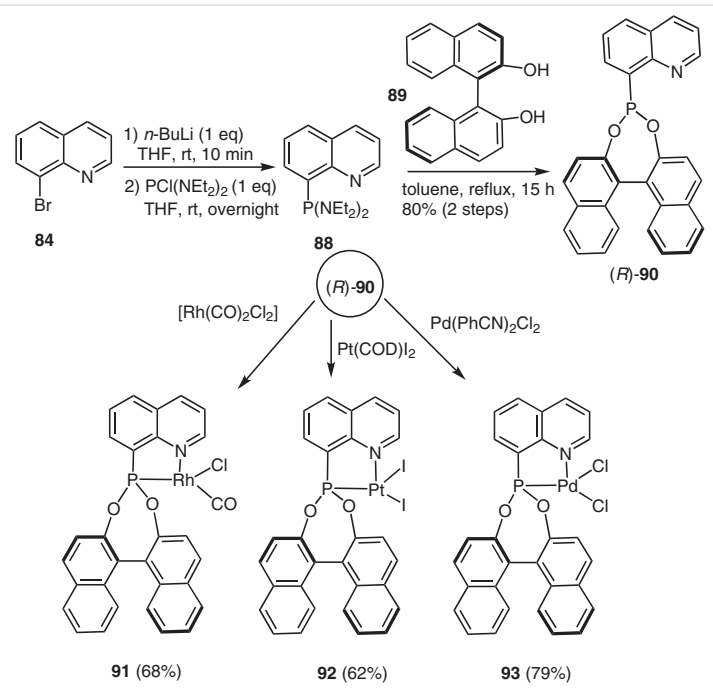

Scheme 19 Synthesis of N,P,O-chiral quinoline-phosphine ligand (R)90 and its $\mathrm{Pd}, \mathrm{Pt}$, Rh complexes ${ }^{86}$

In 2000, Faraone and Leitner introduced the enantioselective synthesis of phosphane/phosphoramidite ligands 96a and 96a' in a one-pot procedure from readily available 8-biarylphosphinoquinoline $\mathbf{9 4}$ by nucleophilic addition of organometallic lithium reagents and direct quenching with $\mathrm{PCl}_{3}$ to obtain $\mathrm{P}, \mathrm{N}$-ligand $\mathbf{9 5}$, followed by addition to chiral 1,1'-bi-2-naphthol 89 in the presence of $\mathrm{Et}_{3} \mathrm{~N}$. Under the same reaction conditions, a 1:1 mixture of diastereomers containing 2-substituted quinoline ligands 99a-d and 99a'd' was obtained from phosphinoquinoline 94. Selected examples are illustrated in Scheme 20.

Knochel and co-workers examined the synthesis of P,Nligands 104 from commercially available starting materials. Treatment of (+)-camphor $\mathbf{1 0 0}$ with $\mathrm{Tf}_{2} \mathrm{NPh}$ in THF at $0{ }^{\circ} \mathrm{C}$ produced the desired compound 101 in 90\% yield (Scheme 21). The chiral camphor triflate $\mathbf{1 0 1}$ efficiently underwent a Pd-catalyzed Negishi cross-coupling reaction with the quinoline organozinc reagent, ${ }^{88 \mathrm{a}-\mathrm{d}}$ affording the desired 2alkenylquinoline $\mathbf{1 0 2}$ in acceptable yield. Subsequent hydrophosphination with $\mathrm{Ph}_{2} \mathrm{P}(\mathrm{O}) \mathrm{H}$, in the presence of a catalytic amount of $t$-BuOK (20 mol\%) in DMSO provided phosphine oxide 103 (Scheme 21). Reduction of compound 103 was accomplished in the presence of $\mathrm{HSiCl}_{3}$ and $\mathrm{Et}_{3} \mathrm{~N}$ in toluene at reflux, to generate the chiral aminophosphine $\mathbf{1 0 4}$ in good yield.

Chiral Ir complex 105 was synthesized by reaction of $[\operatorname{Ir}(\operatorname{cod}) \mathrm{Cl}]_{2}$ and $\mathrm{P}, \mathrm{N}$-ligand $\mathbf{1 0 4}$ in DCM at reflux. After treatment with $\mathrm{NaBAr}_{\mathrm{F}}$ in a biphasic DCM- $\mathrm{H}_{2} \mathrm{O}$ system, the subsequent orange colored salt $\mathbf{1 0 5}$ was obtained after chromatographic purification. The iridium chiral metal complexes were stable towards moisture and oxygen.

Jiang et al. have designed and synthesized a series of phosphine-quinoline ligands. Their synthetic protocol began from optically pure paracyclophane 106. Hence, treat-

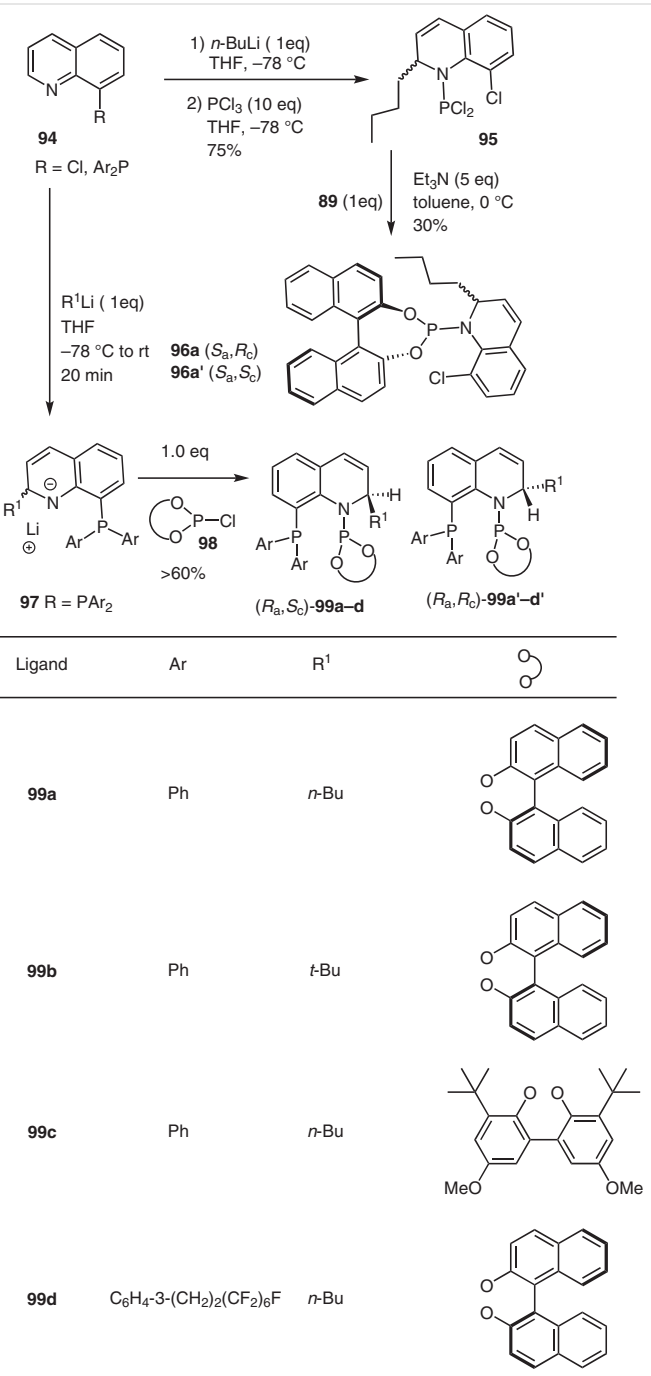

Scheme 20 Synthesis of chiral phosphane/phosphoramidite ligands ${ }^{87}$

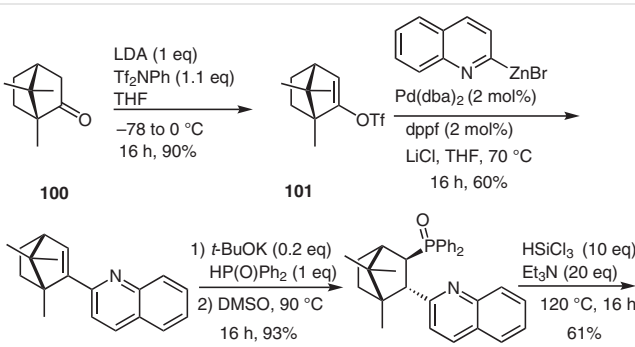

102

103

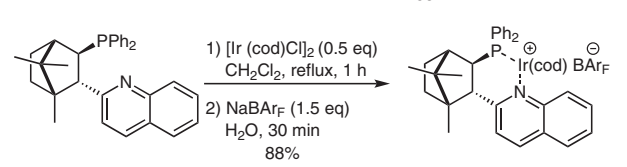

104

105

Scheme 21 Synthesis of a chiral $P, N$-quinolinyl ligand and its Ir complexes $^{88, f}$ 
ment of $\left(R_{p}\right)$-106 with $n$-butyllithium ( $n$-BuLi) followed by successive addition to 2-quinolinylcarboxaldehyde, produced two diastereoisomers, $\left(S_{p}, S\right)-107 \mathbf{a}^{\prime}$ and $\left(S_{p}, R\right)$-107a that could be readily separated by flash column chromatography (Scheme 22). Modifying (Sp,S)-107a' and $(S p, R)-\mathbf{1 0 7} \mathbf{a}$ by silylation in the presence of TBSOTf and lutidine as a base produced $(S p, S)-\mathbf{1 0 8} \mathbf{a}^{\prime}$ in $98 \%$ yield and $(S p, R)-\mathbf{1 0 8}$ in $97 \%$ yield, respectively.

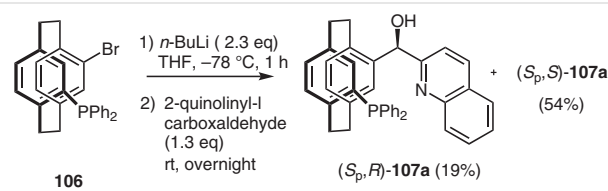

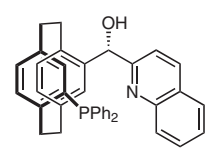

$107 a^{\prime}$ or $107 a$

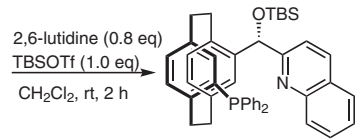
$\left(S_{p}, R\right)-108 \mathbf{a}(97 \%)$
$\left(S_{p}, S\right)-108 \mathbf{a}^{\prime}(98 \%)$
Scheme 22 Synthesis of chiral phosphino-quinoline paracyclophane $\mathrm{P}, \mathrm{N}$-ligands ${ }^{89}$

Ruzzicon et al. investigated the valuable synthesis of $\mathrm{P}, \mathrm{N}$-bidentate planar chiral ligands 111 and 114. Deprotonation of methyl compound 109 with $n$-BuLi at $0{ }^{\circ} \mathrm{C}$, involved the 2-methyl quinoline, giving the 2-methyllithium intermediate, exclusively. The borane complex $(R)-\mathbf{1 1 0}$ was achieved in high yield, by the reaction of $\mathrm{Ph}_{2} \mathrm{PCl}$ with $\mathrm{BH}_{3} \cdot \mathrm{OMe}_{2}$ (Scheme 23). The subsequent air-stable borane complex $(R)-\mathbf{1 1 0}$ was treated with DABCO, to obtain the expected phosphine $(R)$-111. On the other hand, bromo-compound 113 was prepared from alcohol 112 by treating with $\mathrm{CBr}_{4}$ and $\mathrm{PPh}_{3}$ in $\mathrm{Et}_{2} \mathrm{O}$ at $25{ }^{\circ} \mathrm{C}$ and successfully underwent nucleophilic substitution with lithium (diphenylphosphine)methylborane complex, followed by treatment with DABCO, providing the corresponding P,N-chiral ligand $(R)$ 114 in $70 \%$ overall yield (Scheme 23 ).

Brown et al. focused on the synthesis of chiral ligands 121a-g (QUINAP). Boronic acid 116 underwent smooth cross-coupling with aryl chloride $\mathbf{1 1 5}$ in the presence of 3 $\mathrm{mol} \% \mathrm{Pd}\left(\mathrm{PPh}_{3}\right)_{4}$ and $\mathrm{Na}_{2} \mathrm{CO}_{3}$ in DME to give carbon-carbon coupled product $\mathbf{1 1 7}$ in $96 \%$ yield. Cleavage of the methyl group from aryl methyl ether $\mathbf{1 1 7}$ with boron tribromide $\left(\mathrm{BBr}_{3}\right)$ gave the required phenol analogue 118, which was further converted into the triflate $\mathbf{1 1 9}$ (Scheme 24). Finally, palladium-catalyzed cross-coupling of triflate $\mathbf{1 1 9}$ with diphenylphosphine oxide gave the phosphine oxide $\mathbf{1 2 0}$. Subsequently, compound $\mathbf{1 2 0}$ was reduced to the phosphine ligand 121 with $\mathrm{HSiCl}_{3}$ and $\mathrm{Et}_{3} \mathrm{~N}$ in $84 \%$ yield.

Finally, the racemic ligand $\mathbf{1 2 1}$ was reacted with the chiral palladacycle $\mathbf{1 2 2}$ to form diastereomers, from which the desired enantiopure $R$ or $S$ ligands $\mathbf{1 2 1 a}-\mathbf{g}$ were obtained in good yields after fractional recrystallization and ligand decomplexation (Scheme 24).
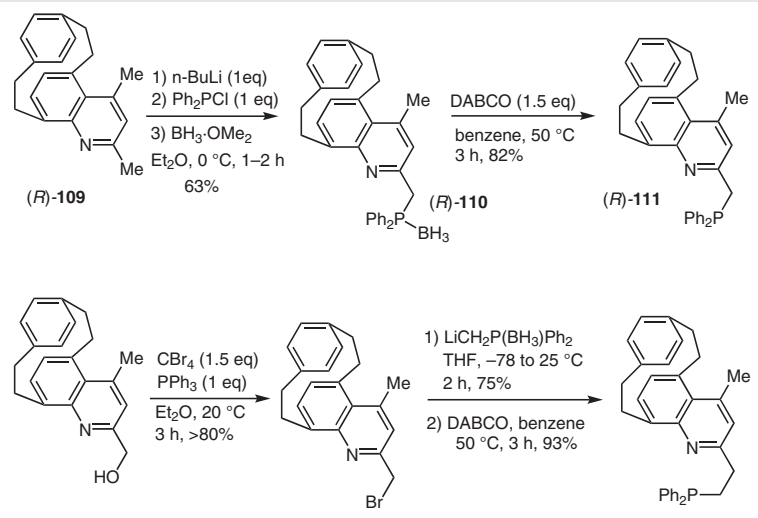

$(R)-112$

(R) -113

$(R)-114$

Scheme 23 Synthesis of P,N-planar chiral ligands ${ }^{90}$
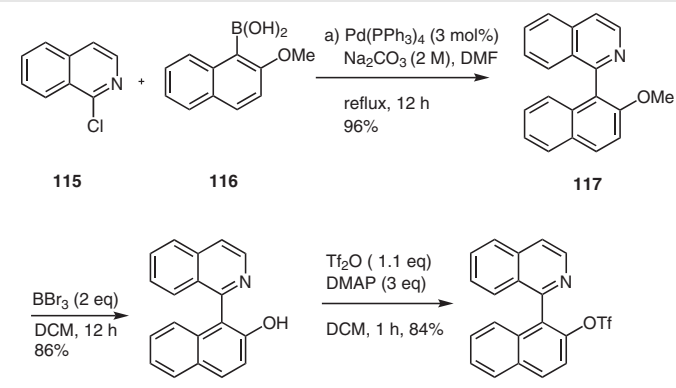

118

119

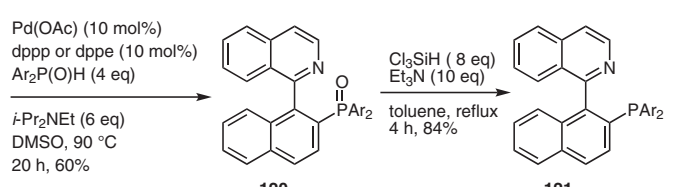

120

121

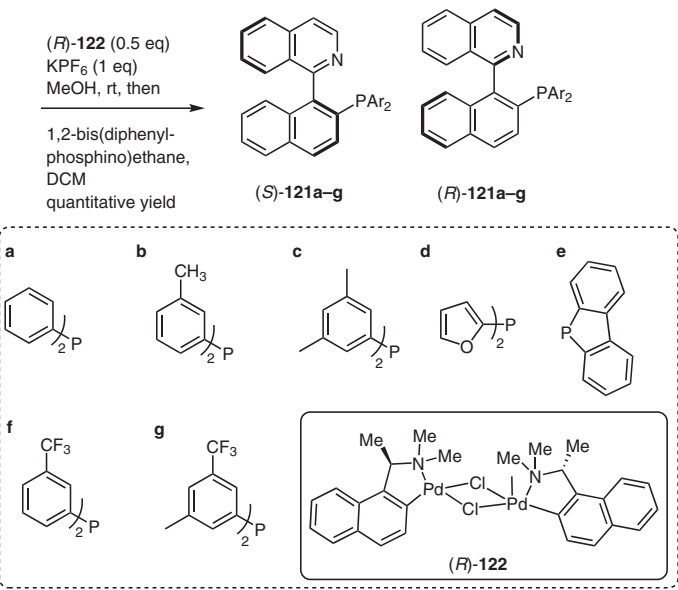

Scheme 24 Synthesis of $P, N$-isoquinoline chiral ligand ${ }^{91,75}$ 
Furthermore, the same group developed a method for the preparation of benzo ring fused isoquinoline and indole-based chiral P,N-ligands 128 and 134 (Scheme 25 and Scheme 26).

Ding and co-workers have synthesized spiro-based P,Nligand 146 through a sequence of reactions as shown in Scheme 27. Nucleophilic addition of compound 136, to a ketal derivative $\mathbf{1 3 7}$ generated a protected spiro-diketone 138. Then Friedländer condensation of $\mathbf{1 3 9}$ with 2-amino benzaldehyde 140 in the presence of $\mathrm{KOH}$ and EtOH furnished the polycyclic quinoline $\mathbf{1 4 1}$ in $70 \%$ yield. Selective deprotection of compound 141 in aq. TFA at room temperature for $1 \mathrm{~h}$ furnished the corresponding spiro-ketone $\mathbf{1 4 2}$
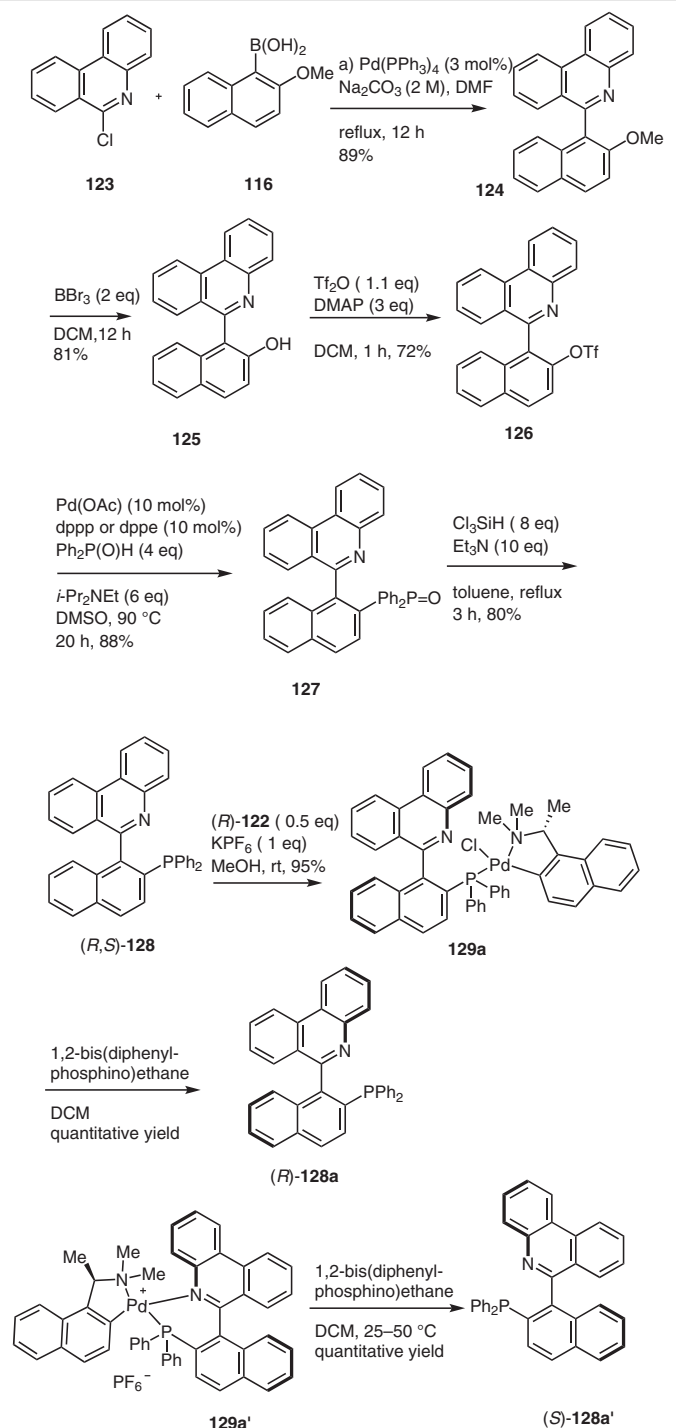

Scheme 25 Synthesis of $P, N$-benzo ring-fused isoquinoline chiral ligand $^{91}$

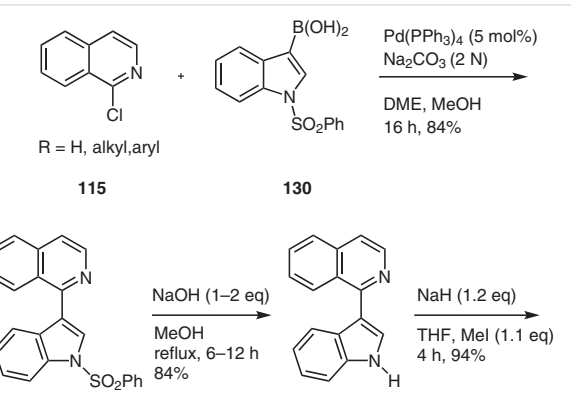

131

132

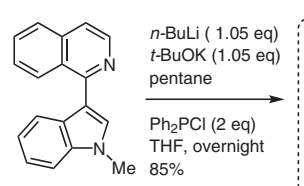

133

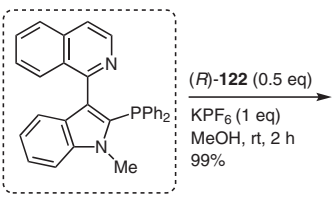

134

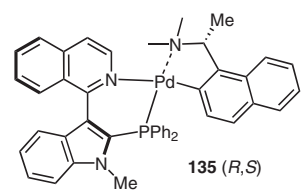

1,2-bis(diphenyl- $\quad(R)-134$ $\underset{\text { phosphino)ethane }}{\longrightarrow} \stackrel{(R)-134}{+}$ $\begin{array}{lll}\mathrm{DCM}, 25-50^{\circ} \mathrm{C} & (S)-134\end{array}$

Scheme 26 Synthesis of chiral P,N-ligands with an indole unit ${ }^{91}$
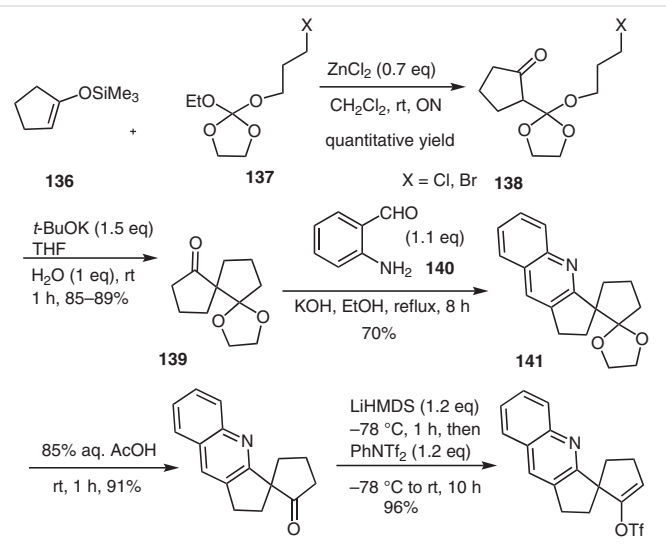

142

143
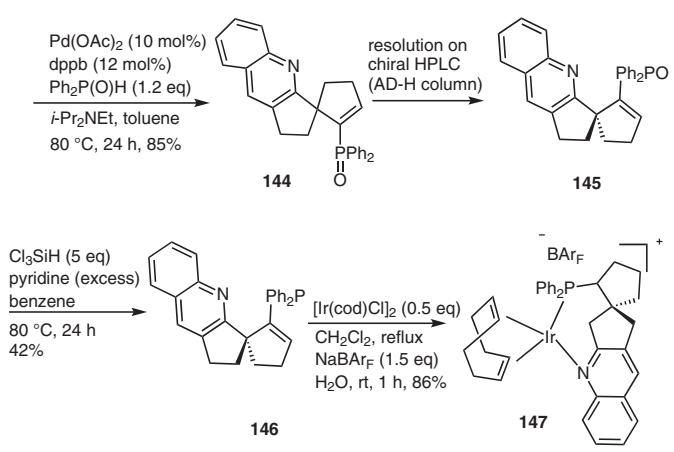

Scheme 27 Synthesis of chiral P,N-ligands with a spiro-skeleton ${ }^{92}$ 
in excellent yield. Subsequent treatment of spiro-compound 142 with LiHMDS, followed by addition of $\mathrm{PhNTf}_{2}$, gave enol triflate 143 in $96 \%$ yield. Next, the coupling reaction of compound 143 with $\mathrm{Ph}_{2} \mathrm{P}(\mathrm{O}) \mathrm{H}$ in the presence of Pd catalyst afforded racemic phosphine oxide 144 in $85 \%$ yield, which was readily resolved by chiral HPLC to give both enantiomers in enantiomerically pure form.

The resulting chiral phosphine oxide $\mathbf{1 4 5}$ was simply reduced with $\mathrm{HSiCl}_{3}$ in the presence of pyridine, affording the required chiral nitrogen ligand (S)-146 in moderate yield (Scheme 27). The reaction of nitrogen based P,N-ligand 146 with $[\operatorname{Ir}(\operatorname{cod})-\mathrm{Cl}]_{2}$ in DCM followed by addition of NaBArF after counter-anion exchange gave the corresponding desired Ir metal complex (+)-147 in $87 \%$ yield.

Multi-step synthesis of silyl substituted chiral quinolinyl phosphane ligands 153a-c has been achieved by Pfaltz and co-workers. In the initial step, hydroxylation of compound 148 using a metal catalyst gave the corresponding 1,2-diol 149 in moderate yield and high enantiomeric excess on a gram scale ( $46 \%$ yield, $94 \%$ ee). Selective tosylation of the primary alcohol 149 in the presence of TsCl with pyridine, followed by silylation of benzyl alcohol $\mathbf{1 5 0}$ using TBDMSCl and imidazole generated enantiomerically pure compound 151 after recrystallization.

Next, sulfonate 151 was treated with $\mathrm{LiPP}_{2} \cdot \mathrm{BH}_{3}$ at $-78^{\circ} \mathrm{C}$ to furnish the phosphine-protected ligand $\mathbf{1 5 2}$ in good yield (Scheme 28). Finally, the P-B bond was successfully cleaved using diethylamine to afford the desired $\mathrm{P}, \mathrm{N}$-ligands 153a-c in good yield.
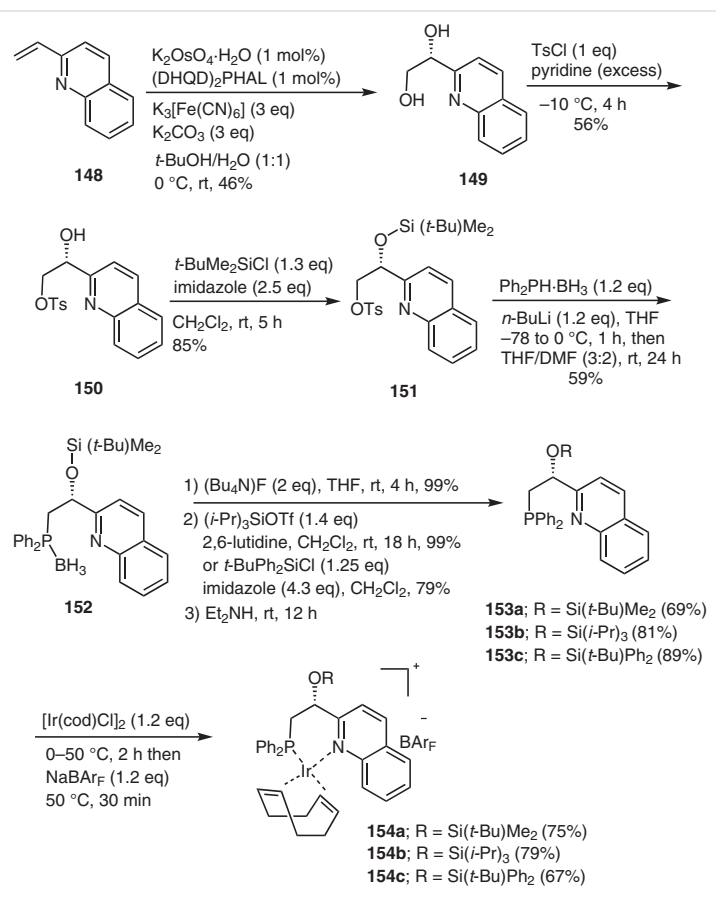

Scheme 28 Synthesis of silyl substituted chiral quinolinyl phosphane ligands and their Ir complexes ${ }^{80,93}$
Additionally, Ir-based transition-metal complexes 154a-c were produced from $N$-heteroaryl phosphane derivatives 153a-c. Warming a DCM solution of the requisite organocatalysts 153 in the presence of $[\operatorname{Ir}(\operatorname{cod}) \mathrm{Cl}]_{2}(0.5$ equiv) for $2 \mathrm{~h}$ at $30-40{ }^{\circ} \mathrm{C}$ followed by counter-ion exchange with $\mathrm{NaBArF}$ (1.6 equiv), provided the metal complexes as orange solids. These types of metal complexes are generally stable to air and moisture, and are simply purified by flash column chromatography on silica gel (Scheme 28).

Chelucci et al. reported a new class of bidentate ligands 160, 164 and 168 that were synthesized from the corresponding starting materials $(+)$-nopinone, $(+)$-camphor and 5 -androst-2-en-17-one. The direct lithiation of compound 155 with $t$-BuLi at low temperature and then quenching with electrophile DMF affording coupled aldehyde 156. The
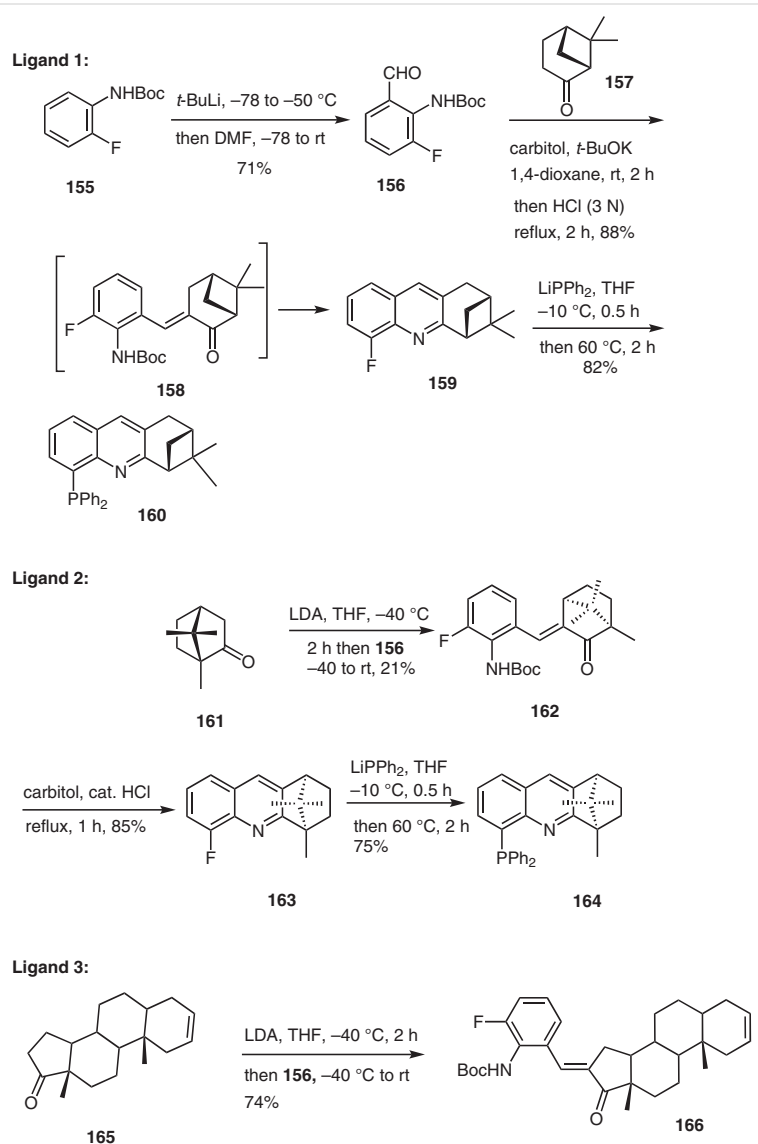

165

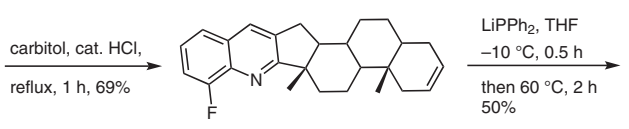

167

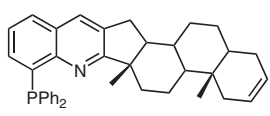

168

Scheme 29 Synthesis of quinoline based P,N-chelating ligands ${ }^{94}$ 
$\mathrm{N}$-Boc aldehyde 156 thus obtained reacts with acyclic ketone $\mathbf{1 5 7}$ in the presence of $t$-BuOK at $25^{\circ} \mathrm{C}$, leading to 159 in good yield (Scheme 29). Finally, treatment of compound 159 with $\mathrm{LiPPh}_{2}$ gave the desired acridine 160 in $82 \%$ yield. The same group used similar reaction conditions to prepare additional quinoline-based P,N-chelating chiral ligands 164 and 168 in good yields (Scheme 29).

Wild and co-workers introduced an efficient method for the preparation of ( $R$ or $S$ )-carbene ligands 173 (Scheme 30). The reaction of halogenated quinoline 169 with $\mathrm{Na}(\mathrm{PMePh})$ in THF furnished the desired compound $\mathbf{1 7 0}$ in very good yield. This racemic product was resolved by crystallization of a pair of internally diastereoisomeric $\mathrm{Pd}(\mathrm{II})$ complexes $(R, R)$ - and $(R, S)$-171a,a' derived from the chelating ligand $(R)-\mathbf{1 2 2}$. The resulting tertiary phosphine $(R)$ and $(S)-\mathbf{1 7 2}$ was accessed by treatment with $\mathrm{H}_{2} \mathrm{SO}_{4}$ and $\mathrm{LiCl}$ (Scheme 30). Finally square-planar palladium complexes $(R)$ - and $(S)$-172 were successfully converted into the optically pure enantiomers $(S)$ - and $(R)-\mathbf{1 7 3}$ with aq. KCN and $\mathrm{DCM} / \mathrm{H}_{2} \mathrm{O}$ in a biphasic reaction medium.
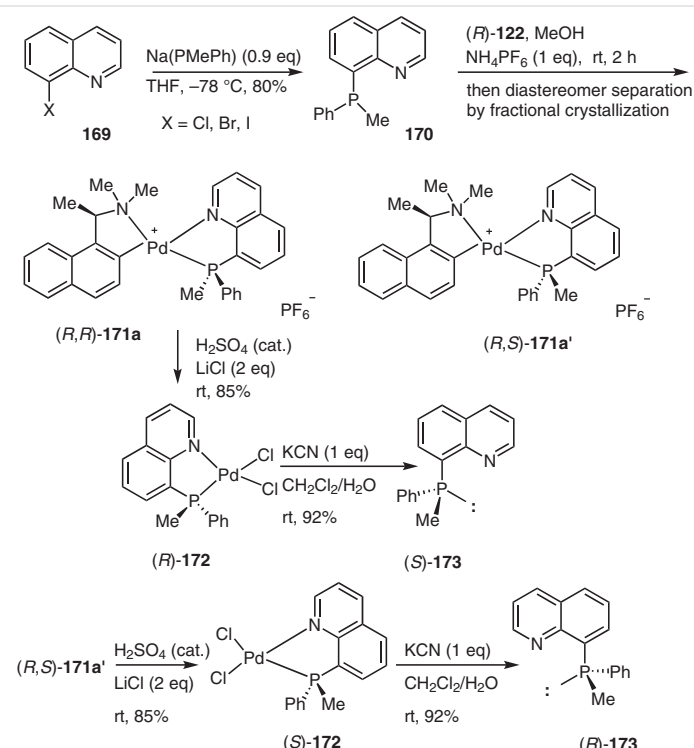

Scheme 30 Synthesis of chiral P,N-carbene ligands ${ }^{75,95}$

These quinoline-based P,N-ligands were broadly applied as asymmetric catalysts in cyclopropanation of olefins, Heck reactions, hydrogenation of olefins, ketones and imines, hydroformylation, allylic alkylation, and oxidative hydroboration.

\subsection{Synthesis of Chiral $\mathrm{N}$-Oxide and Nitrogen Ligands}

Martinez et al. developed an efficient method for the preparation of camphor sulfonamide-based quinoline chiral ligands and their $\mathrm{N}$-oxide derivatives. These chiral amine ligands $174 a-c\left(C_{2}\right.$-symmetry) were prepared by the addi- tion of camphorsulfonyl chloride 70 to 1,2-cyclohexanodiamine 59 and, without additional purification, the resulting intermediates were treated with an aminobenzyl alcohol (Scheme 31) to afford the desired camphor sulfonamidebased quinoline ligands $\mathbf{1 7 4 a - c}$ in high yields. The quinoline $\mathrm{N}$-dioxide ligands $\mathbf{1 7 5} \mathbf{a}-\mathbf{c}$ were simply synthesized from the corresponding ligands $174 a-c\left(C_{2}\right.$-symmetry) by oxidation with $m C P B A$ in DCM at $0{ }^{\circ} \mathrm{C}$. The resulting amine type $\mathrm{N}$-dioxide ligands $\mathbf{1 7 5 a}$-c were formed in reasonable yields and were typically stable enough to be purified by flash column chromatography.
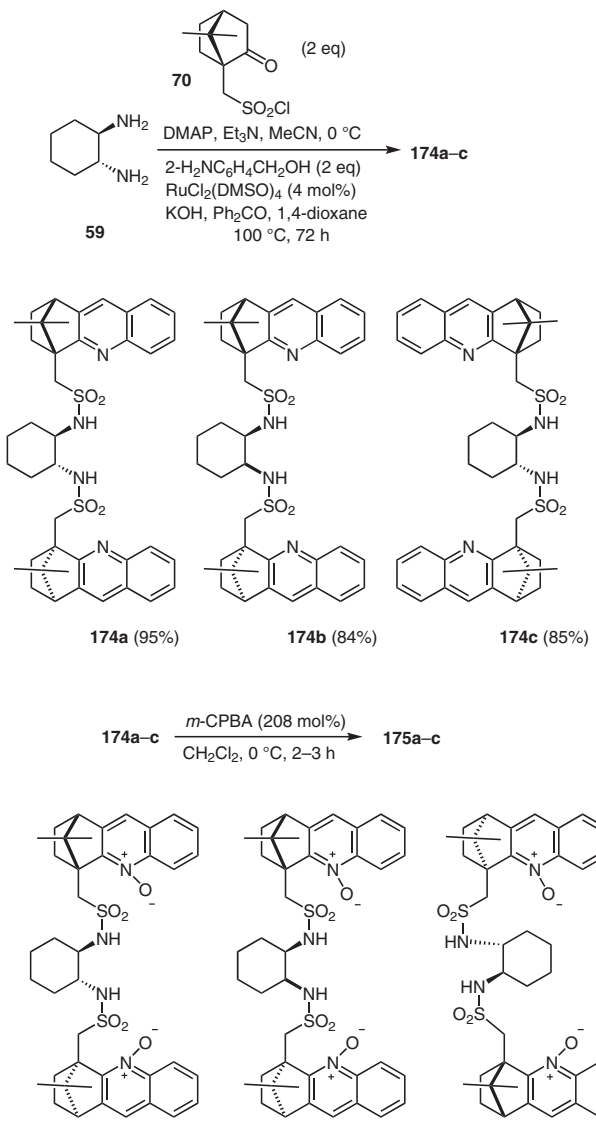

$175 \mathrm{a}(48 \%)$
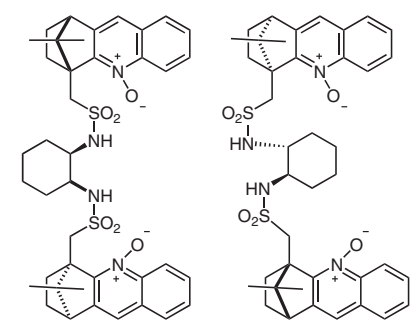

$$
175 b(62 \%)
$$

$175 \mathrm{c}(51 \%)$

Scheme 31 Synthesis of camphorsulfonamide-based quinoline $N, N^{\prime}$ dioxide ligands ${ }^{82}$

Nakajima et al. successfully developed a protocol for the synthesis of $C_{2}$-symmetric 2,2'-biquinoline $N, N^{\prime}$-dioxide $(R$ or $S)-\mathbf{1 7 8}$ and $1,1^{\prime}$-biisoquinoline $N, N^{\prime}$-dioxide ( $R$ or $\left.S\right)$-182 (Scheme 32). The racemic compound 177 was prepared by $m$ CPBA oxidation of 3,3'- dimethyl-2,2'-biquinoline $\mathbf{1 7 6}$, and the product was resolved through a hydrogen-bonding complex with $(S)$ - or $(R)$-binaphthol to afford desired chiral compounds $(R)-\mathbf{1 7 8 a}$ and $(S)-\mathbf{1 7 8} \mathbf{a}^{\prime}$ (Scheme 32). The enantiomerically pure ligand $1,1^{\prime}$-biisoquinoline $N, N^{\prime}$-dioxide 


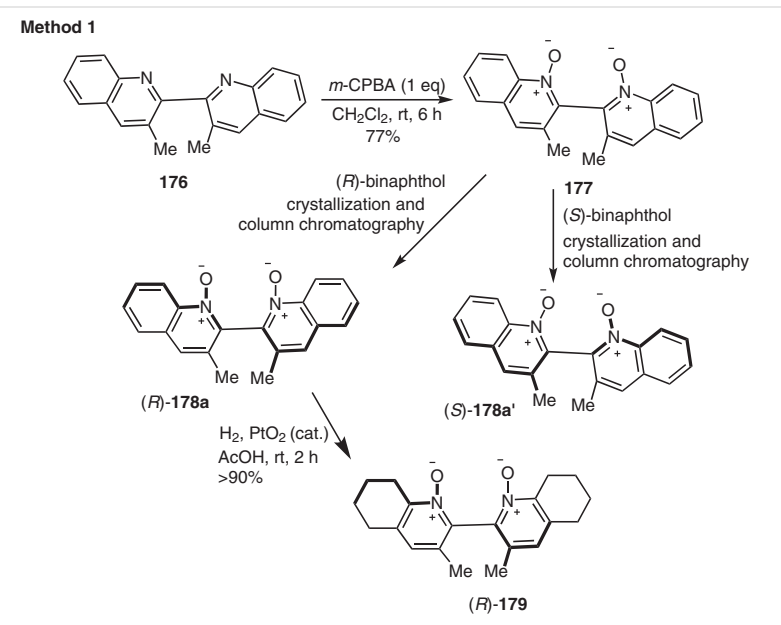

Method 2

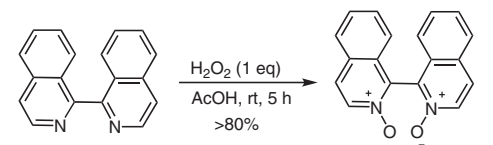

180

181
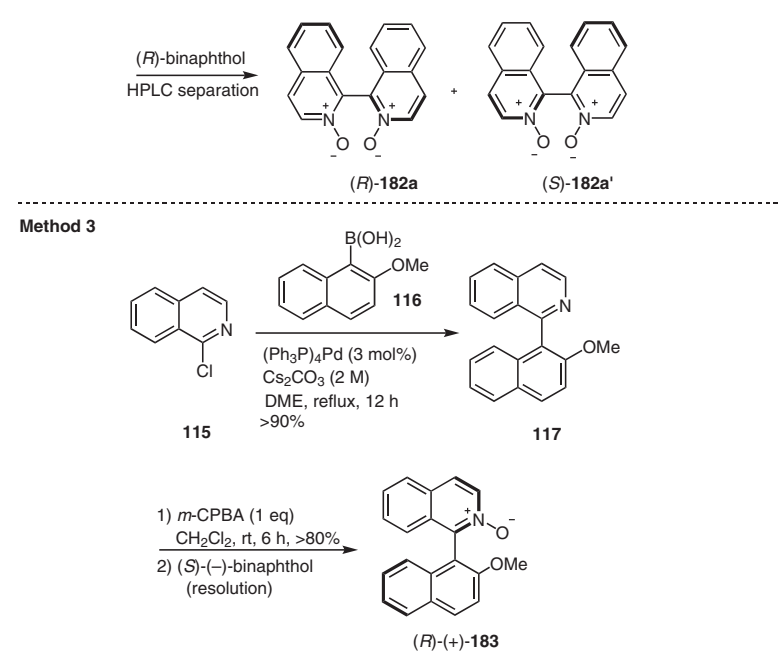

Method 4

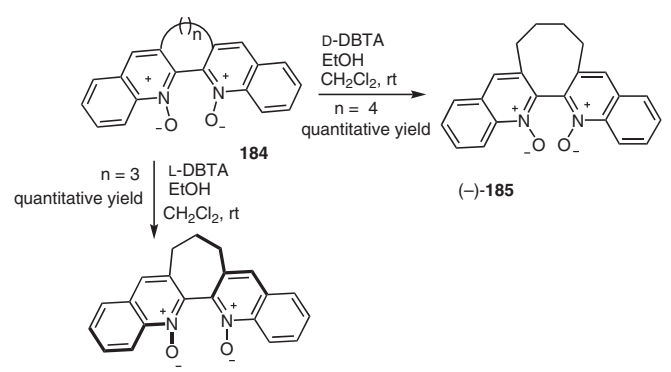

$(+)-186$

Scheme 32 Synthesis of bis-quinoline-based chiral $N, N^{\prime}$-dioxide ligands ${ }^{96}$
(S)-182 was prepared by preparative chiral HPLC from racemic compound 181, which was in turn synthesized by $\mathrm{N}-$ oxidation of 1,10-bisisoquinoline 180 using $\mathrm{H}_{2} \mathrm{O}_{2}$.

The racemic compound $\mathbf{1 1 7}$ was prepared from 1-chloro isoquinoline 115 via Suzuki cross-coupling reaction in the presence of boronic acid 116. Racemic 117 was further reacted with $m C P B A$, and was resolved via a complex with $(S)$-binaphthol to give the required chiral compounds $(R)$ 183.

The ligands (-)-185 and (+)-186 were obtained by resolution of rac-184 with $\mathrm{D}$ and L-dibenzoyltartaric acid, respectively. The absolute configuration of chiral ligand $(S)$ 186 was determined by single-crystal X-ray analysis (Scheme 32). Quinoline-based $N$-oxide ligands were studied in various asymmetric catalytic reactions such as 1,4-addition and Michael addition reactions, allylation of aromatic and heteroaromatic aldehydes, and Strecker reactions.

Finally, in this section, Meyers et al. established the synthesis of chiral naphthylquinoline ligands 188 and 189. Addition of naphthyllithium (1.1 equiv) to quinoline oxazoline 187 in THF at $-78{ }^{\circ} \mathrm{C}$ for $2-3 \mathrm{~h}$ followed by oxidation with dichlorodicyanoquinone (DDQ) in THF at $-78{ }^{\circ} \mathrm{C}$ gave $1-$ naphthyl-4-quinoline $(S)-\mathbf{1 8 8 a}, \mathbf{b}$ in good yield. A similar process using 187 and arylmagnesium reagents ${ }^{97 a}$ followed by oxidation with DDQ $\left(\mathrm{THF},-78{ }^{\circ} \mathrm{C}\right)$ gave the biaryl compounds $(R)-189 a, b$ in high yields (Scheme 33 ).

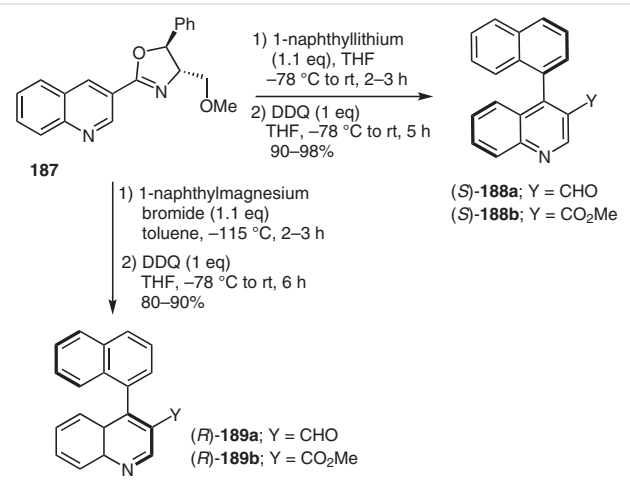

Scheme 33 Synthesis of chiral naphthylquinoline ligands ${ }^{97 b, c}$

\section{Homogeneous Catalytic Asymmetric Re- actions}

\subsection{Asymmetric Carbon-Carbon Bond-Formation Reactions}

Catalytic asymmetric $\mathrm{C}-\mathrm{C}$ bond-forming reactions provide one of the most efficient methods to synthesize chiral molecules, and a range of pyridine and quinoline-based chiral catalysts have been developed in the past two decades, finding a wide range of applications. ${ }^{14-31}$ 


\subsubsection{Asymmetric Addition of Dialkylzinc to Alde- hydes}

In 2008 Cozzi, Yus, Ramón and co-workers described the preparation of camphor sulfonamide-based quinoline ligands. This type of chiral quinoline ligands has been used for the synthesis of trisubstituted chiral alcohols. The enantioselective 1,2-addition of organozinc reagents to substituted aldehydes 190, provides alcohols 191 with high enantioselectivities (up to $96 \%$ ee) with either aromatic or aliphatic substrates (Scheme 34). These reactions were carried out using $10 \mathrm{~mol} \%$ chiral amine ligand 71a organozinc reagent (2.4 equiv) and 1.1 equivalents of $\mathrm{Ti}(\mathrm{O}-i-\mathrm{Pr})_{4}$.

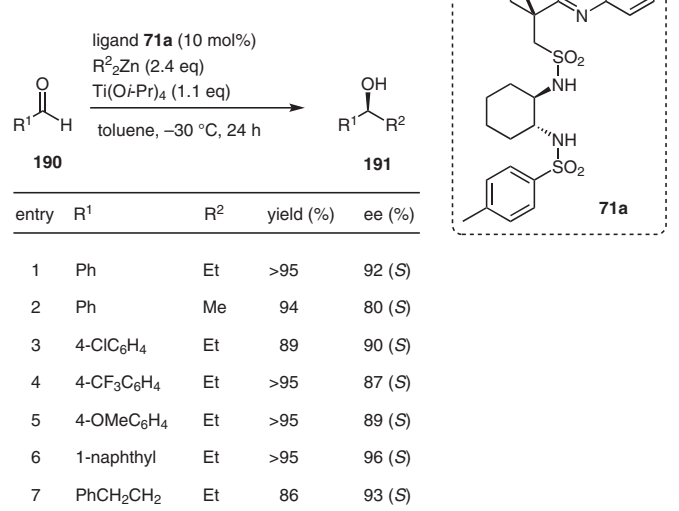

Scheme 34 Enantioselective addition of dialkylzinc reagents to aldehydes $^{82}$

In 2010, Judeh and co-workers synthesized constrained chiral $C_{1}$-symmetric 1,10-bisisoquinoline ligands. The consequences of their geometrical conformations were found to have a significant effect on the catalytic asymmetric addition of diethylzinc to aromatic aldehydes 190. To study the reaction scope and limitations of ligand (+)-67a', several aromatic aldehydes having electron-donating and electron-withdrawing substituents were examined under the optimized reaction conditions. In general, this protocol produced excellent yields and high enantioselectivities of the secondary alcohols 192 (Scheme 35).

\begin{tabular}{|c|c|c|c|}
\hline & \multicolumn{2}{|c|}{$\begin{array}{l}\text { ligand } 67 \mathrm{a}^{\prime}(15 \mathrm{~mol} \%) \\
\mathrm{Et}_{2} \mathrm{Zn}(3.0 \mathrm{eq})\end{array}$} & \\
\hline & $\begin{array}{l}\text { THF/hexane } \\
0^{\circ} \mathrm{C}, 30 \mathrm{~h}\end{array}$ & & \\
\hline entry & $\mathrm{R}^{1}$ & yield (\%) & ee $(\%)$ \\
\hline 1 & $\mathrm{Ph}$ & 96 & 85 \\
\hline 2 & $3-\mathrm{IC}_{6} \mathrm{H}_{4}$ & 89 & 80 \\
\hline 3 & $2,6-\mathrm{Cl}_{2} \mathrm{C}_{6} \mathrm{H}_{3}$ & 99 & 87 \\
\hline 4 & 2-naphthyl & 73 & 81 \\
\hline
\end{tabular}

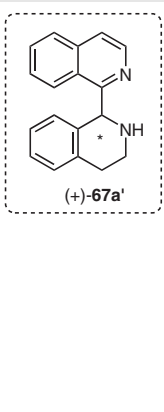

\subsubsection{Asymmetric 1,4-Additions of Dialkylzincs to Enones}

Buono and co-workers investigated the use of a copper catalyst involving QUIPHOS 81a-h as a chiral ligand. This system was applied to the 1,4-addition of $\mathrm{Et}_{2} \mathrm{Zn}$ to $\alpha, \beta$-unsaturated cyclic ketones. Notably, additives such as water or zinc hydroxide had a significant effect, leading to an improved enantiomeric excess from 7 to $61 \%$ ee in this enantioselective 1,4-addition system (Scheme 36).

Faraone and co-workers examined the $\mathrm{Cu}(\mathrm{II})$-catalyzed asymmetric 1,4-addition of diethylzinc to 2-cyclohexen-1one, in the presence of a catalytic amount of chiral ligands 96a and 83h with appropriate metal salts. The 1,4-adducts were formed with enantioselectivities up to $70 \%$ ee with BINAPHOSHQUIN 96a (Scheme 36).

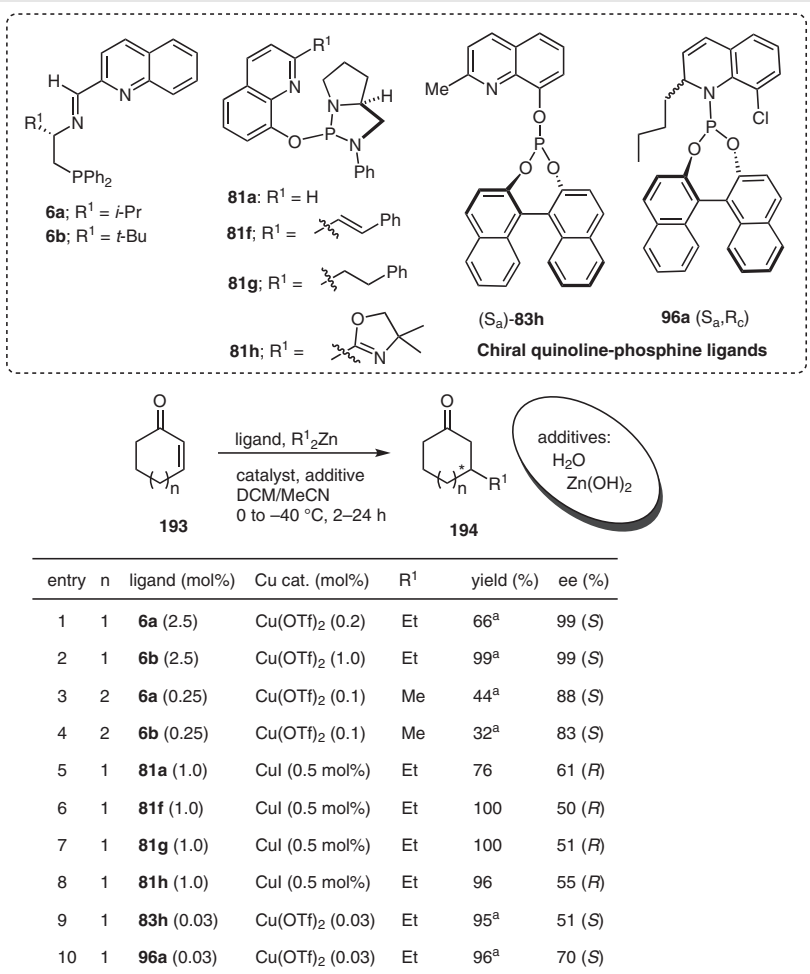

Scheme 36 Enantioselective 1,4-addition of dialkylzinc to cyclic enones $^{68,84,98}$

Later, Hayashi and co-workers developed mild and effective methods for the synthesis of chiral alcohols with excellent enantioselectivity. The copper-catalyzed enantioselective conjugate 1,4 -addition of dialkylzinc reagents to $\alpha, \beta$ unsaturated cyclic ketones 193 with catalytic amounts $(0.2$ $\mathrm{mol} \%$ ) of $\mathrm{Cu}(\mathrm{OTf})_{2}$ and $0.25 \mathrm{~mol} \%$ of one of the N,N,P-tridentate Schiff base ligands 6a,b gave cyclic ketone adducts 194 in up to $99 \%$ ee in good yield (Scheme 36). The impact on the enantioselectivity and reactivity of many other vari-

Scheme 35 Enantioselective addition of diethylzinc to aldehydes ${ }^{81}$ 
ables, such as the nature of the metal catalyst, ligands, and ligand/catalyst loading involved were also examined in detail and the results obtained are summarized in Scheme 36.

Moreover, Hayashi and co-workers further expanded the scope of the 1,4-addition reaction to access disubstituted ketones via copper-catalyzed 1,4-addition of dialkylzincs to $\alpha, \beta$-unsaturated ketones. The reactive zinc enolate intermediates were trapped efficiently with reactive allyl iodides to afford the corresponding disubstituted ketones 195 with excellent diastereo- and enantioselectivity. The desired 1,4-addition reactions were performed using $1 \mathrm{~mol} \% \mathrm{Cu}(\mathrm{OTf})_{2}$ and $1.5 \mathrm{~mol} \%$ Schiff base ligand $\mathbf{6 a}, \mathbf{b}$. The results obtained are summarized in Scheme 37.

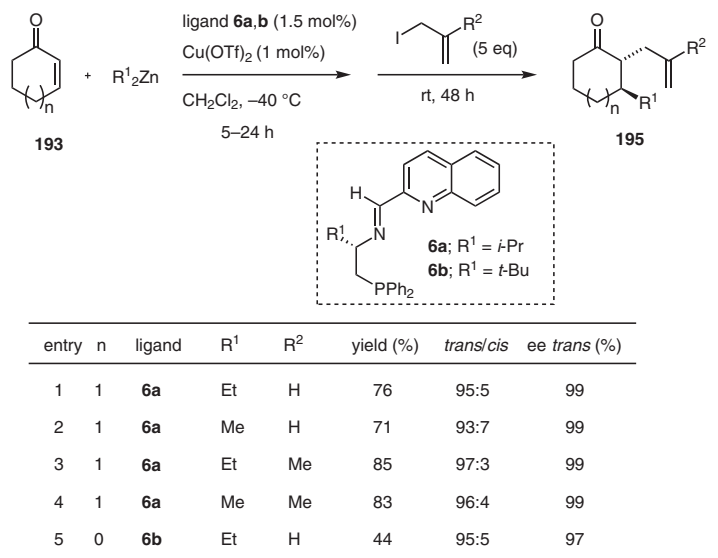

Scheme 37 Enantioselective 1,4-addition followed by trapping of zinc enolate by allyl iodides ${ }^{68 b}$

\subsubsection{Asymmetric Conjugate Addition of Grignard Re- agents to Enones}

Highly constrained $C_{1}-1,10$-bisisoquinoline chiral ligands $(+) /(-)-67$ were examined in the enantioselective 1,4-addition of Grignard reagents ( $\left.\mathrm{R}^{1} \mathrm{MgX}\right)$ to cyclic enones 193. The desired 1,4-adducts 194 were obtained in very good yields but with low enantioselectivity (up to $35 \%$ ee) (Scheme 38).

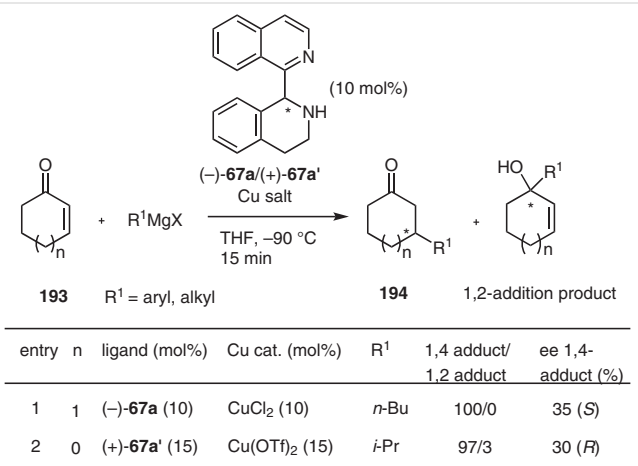

Scheme 38 Grignard reagent conjugate addition to cyclic enones in the presence of copper salts and chiral ligands ${ }^{99}$

\subsubsection{Asymmetric Conjugate 1,4-Addition of Thiols to Cyclic Enones}

Nakajima and co-workers examined the enantioselective conjugate nucleophilic 1,4-addition of thiols to enones under mild reaction condition, leading to the corresponding sulfides 196 with moderate enantioselectivities (up to 78\% ee). This protocol provided the first example of using a cadmium complex in an asymmetric thiol 1,4-addition reaction (Scheme 39).

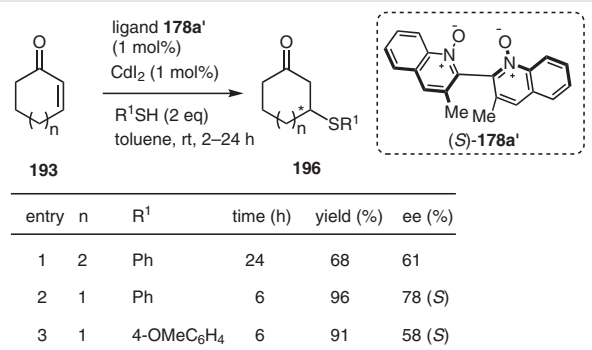

Scheme 39 Enantioselective conjugate 1,4-addition of thiols to enones ${ }^{96 c, d}$

\subsubsection{Asymmetric Michael Addition Reaction}

In 2003, Nakajima et al. studied the catalytic, enantioselective Michael addition of $\beta$-keto esters to $\alpha, \beta$-unsaturated carbonyl compounds using a chiral biquinoline $N, N^{\prime}$-dioxide- $\mathrm{Sc}(\mathrm{OTf})_{3}(R)$-178a complex as catalyst. The Michael adducts 198 were produced in good yields with moderate enantioselectivities (up to 84\% ee) (Scheme 40). Electrondonating indanone substrates 197 were tested using $5 \mathrm{~mol} \%$ quinoline $N, N^{\prime}$-dioxide ligand-Sc(OTf $)_{3}$.

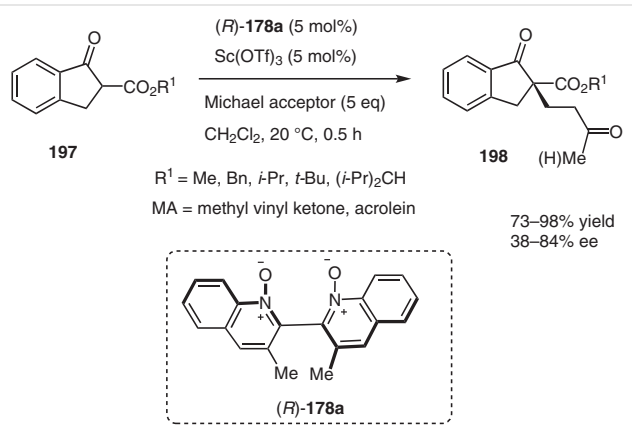

Scheme 40 Enantioselective Michael addition of $\beta$-keto esters to methyl vinyl ketone (MVK) $96 e, 100$

\subsubsection{Asymmetric Friedel-Crafts Alkylation}

Zhou and co-workers (2006) investigated an efficient asymmetric Friedel-Crafts alkylation of free N-H indoles 199 with nitro compound 200 catalyzed by $\mathrm{Zn}(\mathrm{OTf})_{2}$-oxazoline complexes 28e. The nitroindole $\mathbf{2 0 1}$ was prepared in 
good yield, but very low enantioselectivities (up to $9 \%$ ee) were observed in the presence of $12 \mathrm{~mol} \%$ chiral ligand $\mathbf{2 8 e}$ and $10 \mathrm{~mol} \% \mathrm{Zn}(\mathrm{OTf})_{2}$ at $0{ }^{\circ} \mathrm{C}$ for $20 \mathrm{~h}$ (Scheme 41 ).

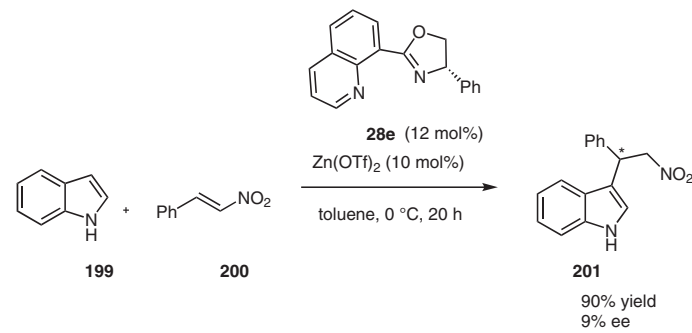

Scheme 41 Asymmetric catalytic Friedel-Crafts alkylation of indole with trans- $\beta$-nitrostyrene ${ }^{101}$

\subsection{Asymmetric Allylic Reactions}

\subsubsection{Asymmetric Allylic Oxidation}

In 2008 Hayashi and co-workers studied the copper (I)catalyzed enantioselective allylic oxidation of several cyclic olefins with tert-butyl perbenzoate $\left(\mathrm{PhCO}_{3} \mathrm{Bu}^{\mathrm{t}}\right)$ enabled by $\mathrm{N}, \mathrm{N}$-bidentate Schiff base ligands 11a, which were effective in conferring high reactivity and moderate-to-good enantioselectivity (up to $84 \%$ ee). The authors examined the allylic oxidation of numerous cyclic olefins 202 using a $\mathrm{Cu}\left(\mathrm{CH}_{3} \mathrm{CN}\right)_{4} \mathrm{PF}_{6}$ and Schiff base ligand system. The results, summarized in Scheme 42, were obtained using a catalytic amount of chiral N,N-bidentate ligand 11a.

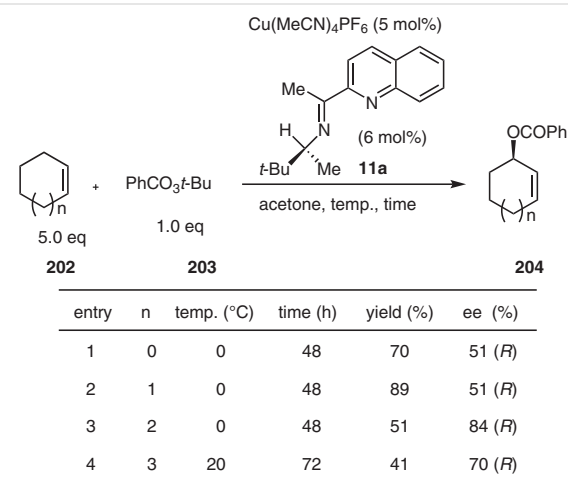

Scheme 42 Enantioselective allylic oxidation of cyclic olefins ${ }^{69}$

Later, in 2009, Hayashi and co-workers developed an enantioselective desymmetrization by allylic oxidation of 4,5epoxycyclohex-1-ene $\mathbf{2 0 5}$ in the presence of $3 \mathrm{~mol} \%$ of chiral N,N-bidentate Schiff base ligand 11a and $2.5 \mathrm{~mol} \%$ of
$\mathrm{Cu}\left(\mathrm{CH}_{3} \mathrm{CN}\right)_{4} \mathrm{PF}_{6}$ to afford phenyl epoxide 206 in $84 \%$ ee, which was improved to $>99 \%$ ee after derivatization with 4 nitro benzoyl chloride and recrystallization to give the corresponding nitroaryl epoxide derivatives 207 (Scheme 43).

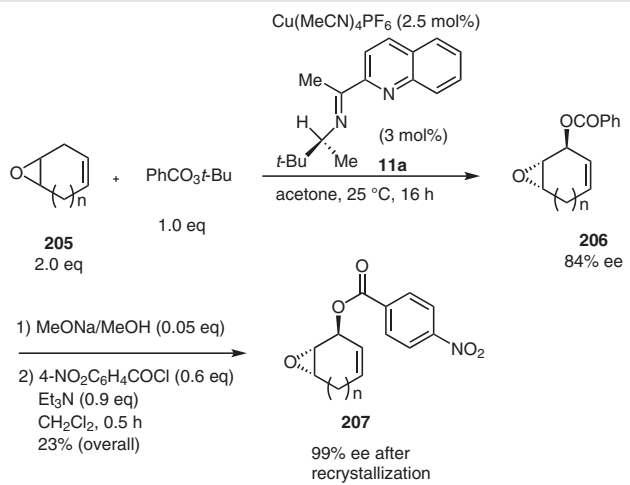

Scheme 43 Enantioselective allylic oxidation of 4,5-epoxycyclohex-1ene $^{102}$

\subsubsection{Asymmetric Allylation of Aldehydes with Allyl- chlorosilanes}

In 2002, Malkov, Koćovský and co-workers developed the Sakurai-Hosomi-type allylation of aromatic aldehydes 190 catalyzed by $C_{2}$-symmetric $2,2^{\prime}$-biquinoline $N, N^{\prime}$-dioxide $(S)-\mathbf{1 7 8}$, leading to the corresponding chiral alcohol 209 in good enantioselectivities (up to $88 \%$ ee) and $85 \%$ reaction yield (Scheme 44)

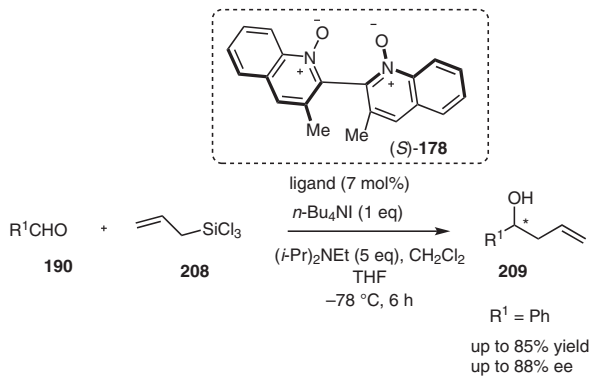

Scheme 44 Sakurai-Hosomi-type allylation of aromatic and heteroaromatic aldehydes ${ }^{103}$

Later, the same group (2003) revealed that the addition of allyltrichlorosilane $\mathbf{2 0 8}$ to aromatic aldehyde $\mathbf{1 9 0}$ in the presence of quinoline $N$-oxide ligand $(R)-\mathbf{1 8 3}(5 \mathrm{~mol} \%)$ at $-40{ }^{\circ} \mathrm{C}$ in DCM for $0.5-12 \mathrm{~h}$, produced the corresponding alcohol derivatives 209 with 5-96\% ee and good yields. The aldehyde substrate scope is summarized in Scheme 45. 


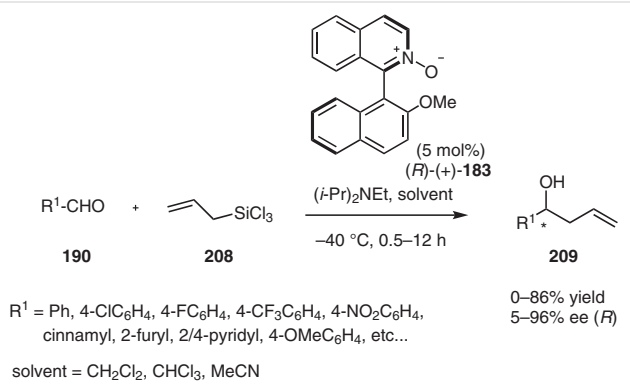

Scheme 45 Allylation of aldehydes catalyzed by quinoline $\mathrm{N}$-oxide ligand ${ }^{80,96 f}$

\subsubsection{Asymmetric Phase-Transfer Allylic Alkylation}

Later, in 2008, Eddine and co-workers reported the phase-transfer-catalyzed asymmetric alkylation of ester $\mathbf{2 1 0}$ with allyl bromide in the presence of $10 \mathrm{~mol} \%$ of chiral $\mathrm{N}, \mathrm{N}$-bidentate Schiff base salt 17 with the use of $\mathrm{NaOH}$, affording the desired compound $\mathbf{2 1 1}$ in good yield and very low enantioselectivity (Scheme 46).<smiles>CC(C)(C)OC(=O)CN=C(c1ccccc1)c1ccccc1</smiles>

210

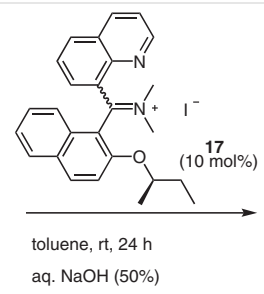

aq. $\mathrm{NaOH}(50 \%)$

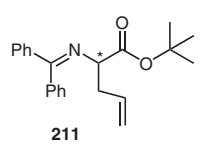

$76 \%$ yield, $5 \%$ ee
Scheme 46 Allylation of keto-imine under phase-transfer conditions ${ }^{71}$

\subsection{Asymmetric Cycloadditions}

\subsubsection{Asymmetric Diels-Alder Reactions}

Buono and co-workers (1998) reported the asymmetric Diels-Alder reaction catalyzed by copper-phosphene complexes. The nitrogen-based copper(II) catalyst was prepared by mixing $\mathrm{Cu}(\mathrm{OTf})_{2}$ and chiral quinolinephosphine ligand 81a in DCM and further used in the Diels-Alder reaction of 3-acryloyloxazolidin-2-one $\mathbf{2 1 3}$ with cyclopentadiene $\mathbf{2 1 2}$, leading to the corresponding amide product 214 in excellent yields and remarkable enantioselectivities (up to 99\%) (Scheme 47).

Subsequently, in 2004, Suga et al. developed an efficient method for $\mathrm{Ni}(\mathrm{II})$-catalyzed asymmetric Diels-Alder reactions of cyclopentadiene $\mathbf{2 1 2}$ and 3-alkenoyl-2-oxazolidinones 215 in the presence of the ligand BINIM-2QN 14a (Scheme 48). Even loadings down to $1 \mathrm{~mol} \% \mathrm{Ni}$ (II) catalyst promoted Diels-Alder reactions with high conversions and enantioselectivities (endo-addition with up to $94 \%$ ee).

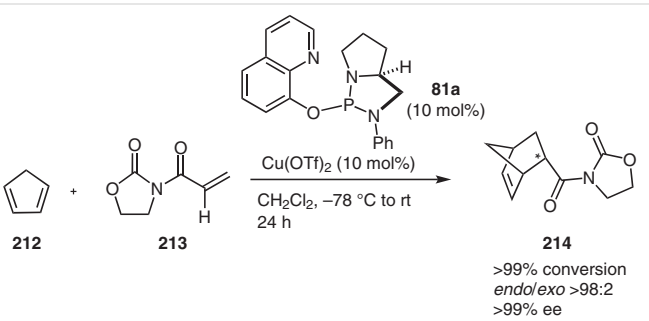

Scheme 47 Copper-catalyzed asymmetric Diels-Alder reactions of cyclopentadiene with $\mathrm{N}$-acyl oxazolidinones ${ }^{104}$

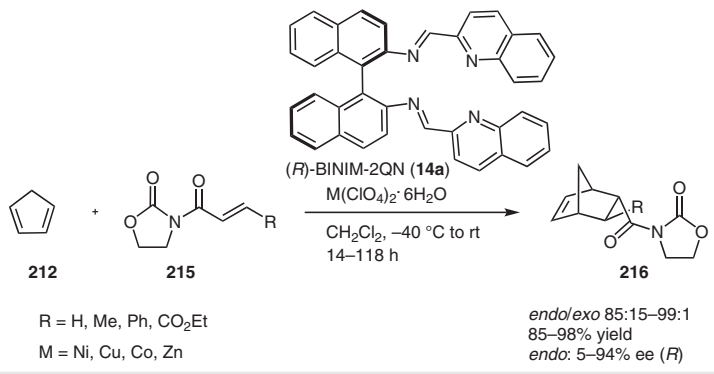

Scheme 48 BINIM-2QN catalyzed asymmetric Diels-Alder reactions of cyclopentadiene with 3-acryloyl-2-oxazolidinone ${ }^{70 a}$

\subsubsection{Asymmetric Hetero-Diels-Alder Reactions}

Bolm et al. studied the first example of a copper-catalyzed hetero-Diels-Alder reaction of cyclohexa-1,3-diene (217) and keto ester 218 in the presence of $10 \mathrm{~mol} \%$ $\mathrm{Cu}(\mathrm{OTf})_{2}$ and $C_{1}$-symmetric sulfoximine ligands 41a-1, leading to cycloadducts in good yields and high enantioselectivities (up to $96 \%$ ee) as shown in Scheme 49.

Asymmetric cycloaddition of nitrones 220 and 3-(2alkenoyl)-2-thiazolidinethiones 221 using chiral binaphthyldiimine-Ni(II) complexes $\mathbf{1 4 a - e}$ to afford products in high exo-diastereoselectivities and enantioselectivities was reported by Suga et al. in 2005 (Scheme 50).
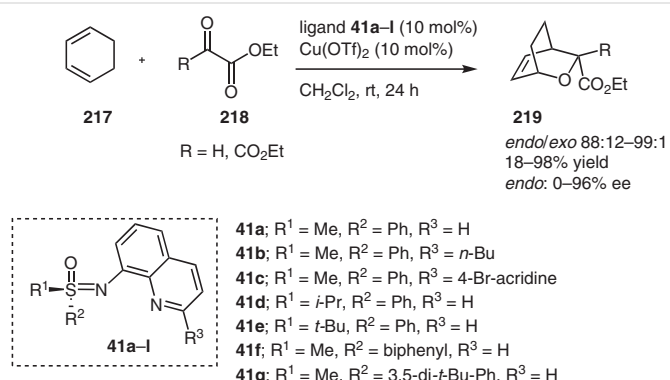

41a; $R^{1}=M e, R^{2}=P h, R^{3}=H$ $41 \mathrm{~b} ; \mathrm{R}^{1}=\mathrm{Me}, \mathrm{R}^{2}=\mathrm{Ph}, \mathrm{R}^{3}=n-\mathrm{Bu}$ 41c; $R^{1}=M e, R^{2}=P h, R^{3}=4-B r$-acridine $41 \mathrm{c} ; \mathrm{R}^{1}=i-\mathrm{Pr}, \mathrm{R}^{2}=\mathrm{Ph}, \mathrm{R}^{3}=\mathrm{H}$
$41, ; \mathrm{R}^{1}=t-\mathrm{Bu}, \mathrm{R}^{2}=\mathrm{Ph}, \mathrm{R}^{3}=\mathrm{H}$

$41 \mathrm{~d} ; \mathrm{R}^{1}=t-\mathrm{Pr}, \mathrm{R}^{2}=\mathrm{Ph}, \mathrm{R}^{3}=\mathrm{H}$
$41 \mathrm{e} ; \mathrm{R}^{1}=t-\mathrm{Bu}, \mathrm{R}^{2}=\mathrm{Ph}, \mathrm{R}^{3}=\mathrm{H}$ $41 \mathrm{e} ; \mathrm{R}^{1}=t-\mathrm{Bu}, \mathrm{R}^{2}=\mathrm{Ph}, \mathrm{R}^{3}=\mathrm{H}$
$41 \mathrm{f} ; \mathrm{R}^{1}=\mathrm{Me}, \mathrm{R}^{2}=$ biphenyl, $\mathrm{R}^{3}=\mathrm{H}$ $41 \mathrm{~g} ; \mathrm{R}^{1}=\mathrm{Me}, \mathrm{R}^{2}=3,5$-di-t- $\mathrm{Bu}-\mathrm{Ph}, \mathrm{R}^{3}=\mathrm{H}$ $41 \mathrm{~h} ; \mathrm{R}^{1}=\mathrm{Me}, \mathrm{R}^{2}=2-\mathrm{MeO}-\mathrm{Ph}, \mathrm{R}^{3}=\mathrm{H}$ $41 ; ; \mathrm{R}^{1}=n$-pentyl, $\mathrm{R}^{2}=2-\mathrm{MeO}-\mathrm{Ph}, \mathrm{R}^{3}=\mathrm{H}$ $41 \mathrm{j} ; \mathrm{R}^{1}=$ phenethyl, $\mathrm{R}^{2}=2-\mathrm{MeO}-\mathrm{Ph}, \mathrm{R}^{3}=\mathrm{H}$ $41 \mathrm{k}: \mathrm{R}^{1}=t-\mathrm{Bu}, \mathrm{R}^{2}=2-\mathrm{MeO}-\mathrm{Ph}, \mathrm{R}^{3}=\mathrm{H}$ $411 ; R^{1}=t-B u, R^{2}=2-M e O-N a p h, R^{3}=H$

Scheme 49 Enantioselective hetero-Diels-Alder reactions catalyzed by monosulfoximine ligands ${ }^{76}$ 


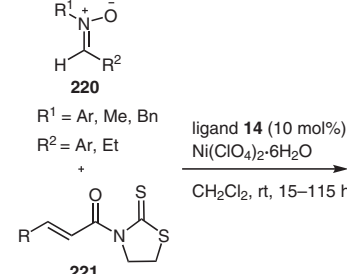
$\mathrm{R}=\mathrm{Me}, \mathrm{Et}, n-\mathrm{Pr}, \mathrm{Ph}$

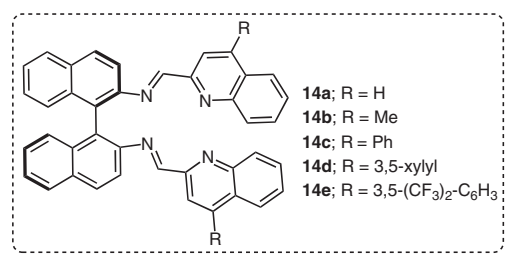

Scheme 50 Asymmetric cycloaddition reactions catalyzed by chiral BINIM-Ni(II) complexes ${ }^{70 b}$

\subsubsection{Asymmetric 1,3-Dipolar Cycloaddition Reac- tions}

Shi and co-workers reported chiral binaphthalenediimine-Ni(II) complex 14a as an active catalyst in the 1,3-dipolar cycloaddition reactions of azomethine ylides 223 and 1-phenyl-1H-pyrrole-2,5-dione 224 to give the corresponding adducts $\mathbf{2 2 5}$ in very low yields and poor enantiomeric excesses (up to 8\%) in Scheme 51.

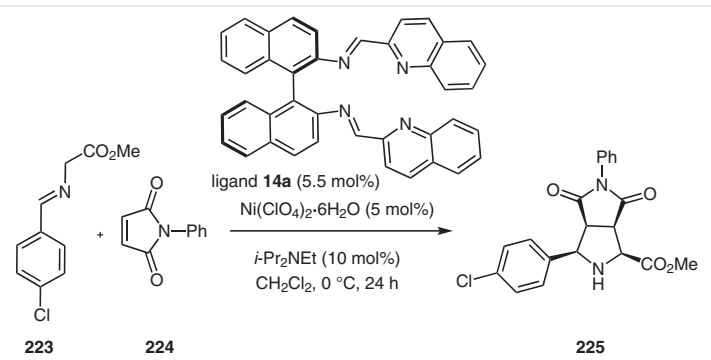

Scheme 51 Enantioselective 1,3-dipolar cycloadditions catalyzed by $\mathrm{Ni}\left(\mathrm{ClO}_{4}\right)_{2} \cdot 6 \mathrm{H}_{2} \mathrm{O}$ and chiral ligands ${ }^{70 c}$

Furthermore, in 2011, Suga et al. demonstrated that BINIM-Ni(II) catalysts $\mathbf{1 4 a - c}$ were efficient for enantioselective 1,3-dipolar cycloaddition reactions between ethyl diazoacetate 226 and 3-acryloyl-2-oxazolidinones 227 to produce the corresponding adducts $\mathbf{2 2 8}$ in high yields and enantiomeric excesses (up to 93\%) as shown in Scheme 52.

The same group had previously reported in 2007 the first example of highly enantioselective 1,3-dipolar cycloaddition reactions between azomethine imines 229 and 3-acryloyl-2-oxazolidinone 230 using $10 \mathrm{~mol} \%$ of chiral BINIM-Ni(II) complex 14b (Scheme 53).

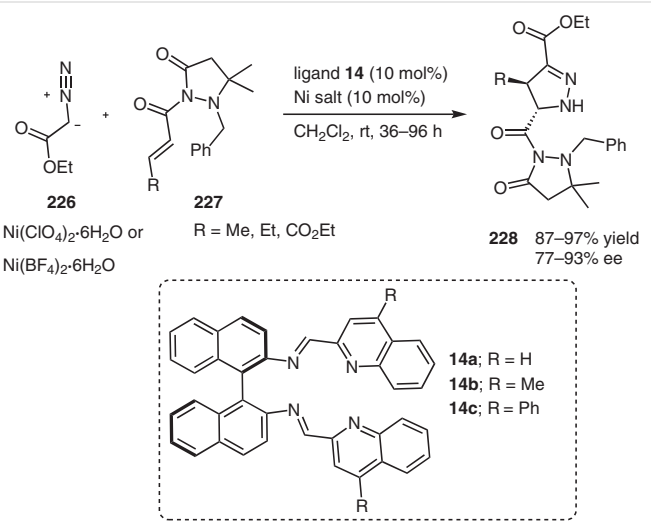

Scheme 52 Reactions of ethyl diazoacetate with 2-(2-alkenoyl)-3-pyrazolidinones catalyzed by $(R)$-BINIM-Ni(II) complexes ${ }^{105}$
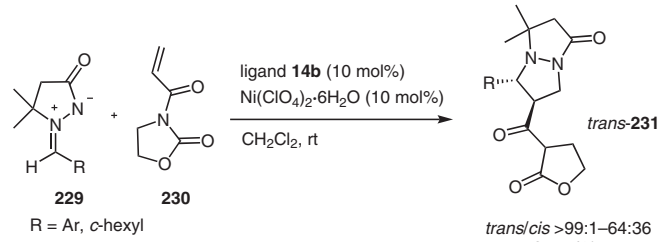

trans/cis >99:1-64:36 trans: $97-74 \%$ ee

Scheme 53 Cycloaddition reactions of azomethine imines with 3-acryloyl-2-oxazolidinone ${ }^{70 d}$

\subsection{Asymmetric Carbene Insertions}

A simple and efficient method for $\mathrm{Cu}$-catalyzed enantioselective $\mathrm{C}-\mathrm{H}$ carbene insertion between methyl phenyldiazoacetate and THF in the presence of $2.2 \mathrm{~mol} \%$ of chiral N,N-bidentate Schiff-base ligand 28 e and $2.0 \mathrm{~mol} \%$ of copper catalyst to afford the corresponding syn-product 234 in 45\% ee, was reported by Fraile et al. in 2007. Copper salts such as $\mathrm{Cu}(\mathrm{OTf})_{2}, \mathrm{CuBr}_{2}, \mathrm{Cu}(\mathrm{OAc})_{2}, \mathrm{CuCl}$, and $\mathrm{CuSbF}_{6}$ were examined to optimize the reaction and copper triflate furnished better results (Scheme 54).

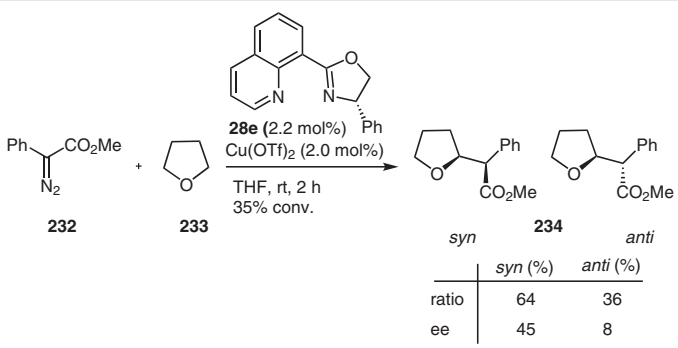

Scheme 54 Reaction between methyl phenyldiazoacetate and THF catalyzed by oxazole-copper complexes ${ }^{106}$ 


\subsection{Asymmetric Pinacol Couplings}

In 2004, Yamamoto and co-workers introduced a new class of chiral tetradentate ligand, ТВOx 52a, as a catalyst for pinacol coupling. Chromium complex $\mathrm{TBOxCrCl} 235$ was shown to be an efficient catalyst for the asymmetric pinacol coupling reactions of both functionalized aromatic and aliphatic aldehydes $\mathbf{1 9 0}$. With aromatic substrates, the catalyst system was shown to be quite insensitive to changes in steric effects on the substrates as well as to the presence of electron-donating and electron-withdrawing substituents on the aromatic ring, providing high enantiomeric excesses (up to $98 \%$, Scheme 55 ).

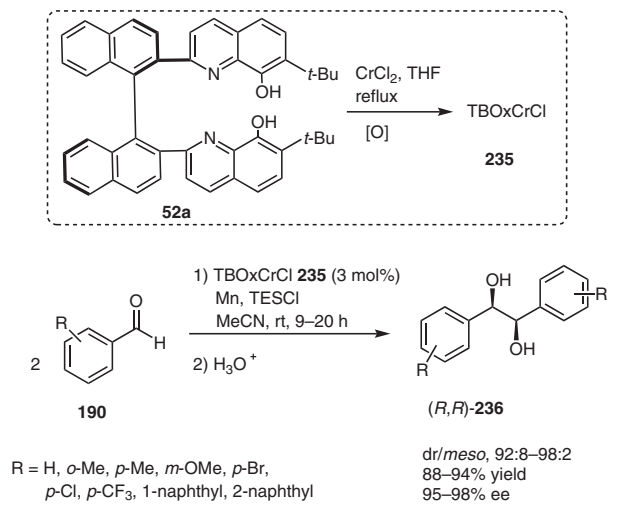

Scheme 55 Pinacol coupling reaction of aromatic aldehydes ${ }^{78 a}$

\subsection{Asymmetric Pudovik Reactions}

The same group in 2008 developed the catalytic enantioselective Pudovik reaction of aldehydes 190 and aldimines 239 with tethered bis(8-quinolinato) (TBOx) aluminum complexes 52a-d. $\alpha$-Hydroxy- and $\alpha$-aminophosphonates 238 and 240 were prepared in high yields and enantioselectivities (96-98\% ee) using a low catalyst loading (1 mol\%). This was a significant improvement over other catalysts in that they generally required higher catalyst loadings, typically $>5 \mathrm{~mol} \%$ and extended reaction times. The chiral ligand could be easily recovered in high purity after simple purification without loss in either reactivity or selectivity (Scheme 56).

\subsection{Asymmetric Strecker Reactions}

Feng and co-workers (2003) investigated enantioselective Strecker reactions with trimethylsilyl cyanide (TMSCN) 242 and aryl imines 241 catalyzed by chiral $N, N^{\prime}$-dioxide ligand 178. These chiral quinoline $N, N$-dioxide Lewis base promoters were effectively applied to the chiral synthesis of $\alpha$-amino aryl nitrile analogues 243 with high enantio-

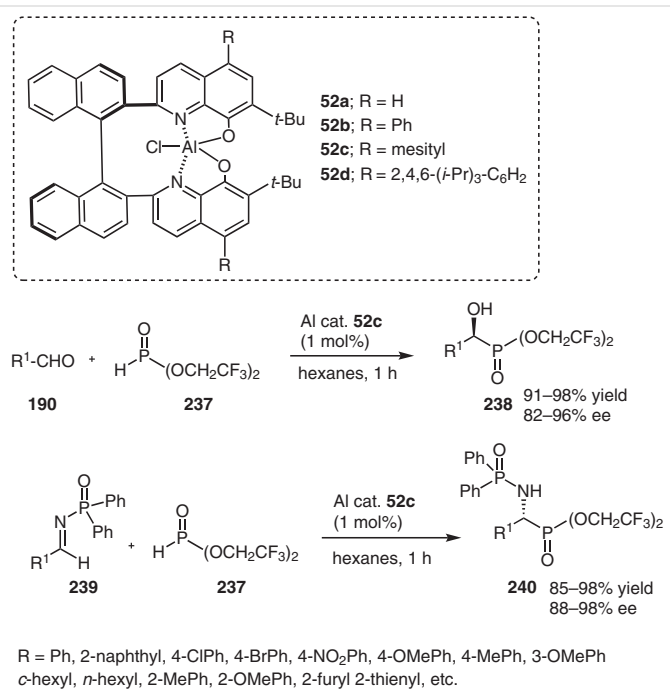

Scheme 56 Catalytic enantioselective Pudovik reaction of aldehydes/aldimines with tethered bis(8-quinolinato) (TBOx) aluminum complexes $^{78 b}$

selectivities (up to 95\% ee). Enantiomerically pure products (up to $99 \% e e$ ) were subsequently obtained by recrystallization (Scheme 57).

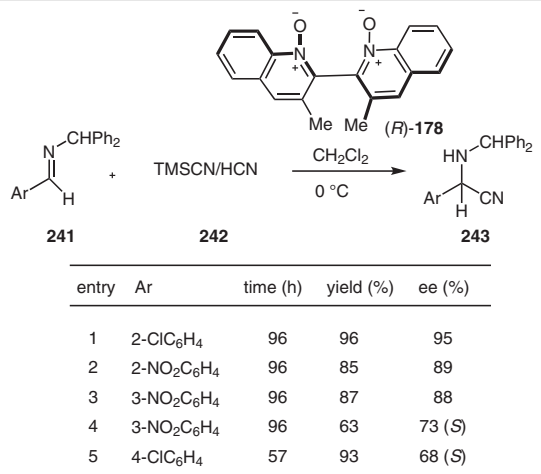

Scheme 57 Enantioselective Strecker reactions between aldimines and $\mathrm{TMSCN}^{96 \mathrm{~g}, \mathrm{~h}}$

\section{Heterogeneous Catalytic Asymmetric Re- actions}

Fraile, Mayoral and co-workers reported quinolinebased oxazoline ligands, a class of $C_{1}$-symmetric chiral ligands, in the enantioselective cyclopropanation of styrene (244) with ethyl diazoacetate 245 in DCM at $25{ }^{\circ} \mathrm{C}$, which proceeded with excellent cis-selectivity (up to $65 \%$ ). This result may be synthetically of interest, given that cis-cyclopropanes are generally difficult to obtain. The substrate scope is summarized in Scheme 58. 


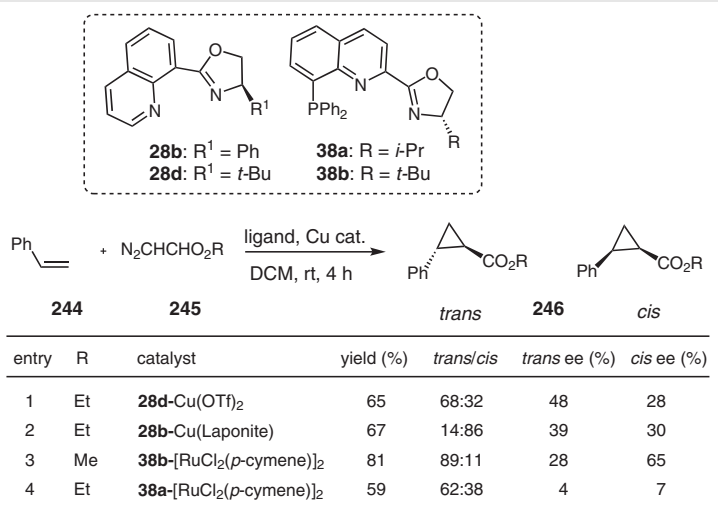

Scheme 58 Cyclopropanation reaction between styrene and diazoacetate esters ${ }^{107}$

\subsection{Asymmetric Cyclopropanation of Olefins}

In 1998, Ahn and co-workers studied the Ru(II)-catalyzed intramolecular cyclopropanation of diazo-alkenes 247. The catalytic chiral system demonstrated good reactivity and stability, and produced high yields with moderate enantioselectivities (Scheme 59).

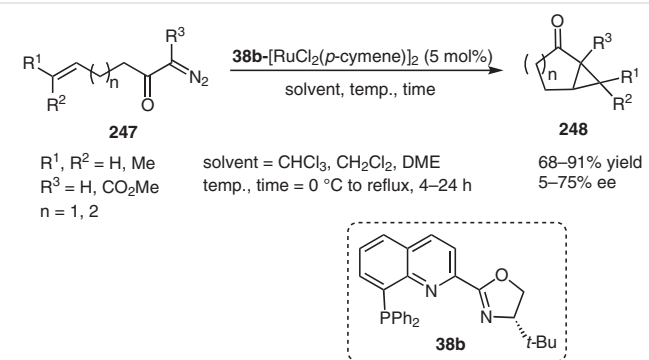

Scheme $59 \mathrm{Ru}(\mathrm{II})$-catalyzed asymmetric intramolecular cyclopropanations using a chiral diphenylphosphino(oxazolinyl)quinoline ligand ${ }^{75 a}$

\subsection{Asymmetric Heck Reactions}

In 2004 Pfaltz and co-workers described the generality and utility of ligands 153a,b in palladium-catalyzed enantioselective Heck reactions. The results are summarized in Scheme 60.

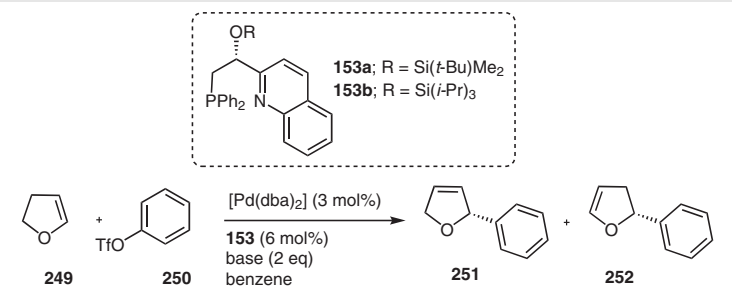

$L=153 a\left(i-P_{2} N E t, 50{ }^{\circ} \mathrm{C}, 2 \mathrm{~d}\right), 97 \%$ ee $(251), 251 / 252=83: 17,100 \%$ conv. $L=153 \mathrm{~b}$ (proton sponge, $50{ }^{\circ} \mathrm{C}, 2 \mathrm{~d}$ ), $99 \%$ ee (251), $251 / 252=95: 5,48 \%$ conv

Scheme 60 Enantioselective Heck reaction using chiral quinolyl phosphane ligands ${ }^{93}$

\subsection{Asymmetric Hydrogenations}

\subsubsection{Asymmetric Hydrogenation of Alkenes}

$\mathrm{P}, \mathrm{N}-\mathrm{Chiral}$ iridium complexes 154a-c were efficiently applied to asymmetric hydrogenation of di-substituted alkenes $\mathbf{2 5 3}$ (Scheme 61, entries 1-3), resulting in up to 56\% ee, as reported by Pfaltz and co-workers in 2004. The reactivities of the metal complexes are summarized in Scheme 61. In general, phosphinites were excellent in terms of both enantioselectivity and reactivity. Additionally, in 2003, Knochel and co-workers demonstrated that ligand 105 mediated Ir-catalyzed asymmetric hydrogenation reactions of tri-substituted alkenes (Scheme 61, entries 4-6) leading to hydrogenated products with high enantioselectivity (up to $95 \%$ ee).

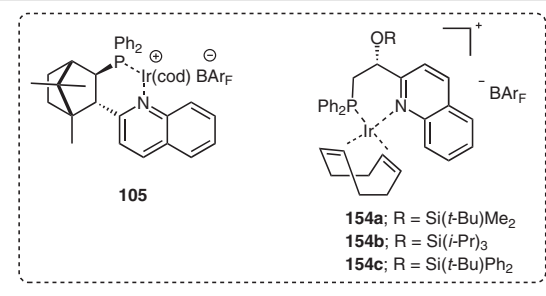

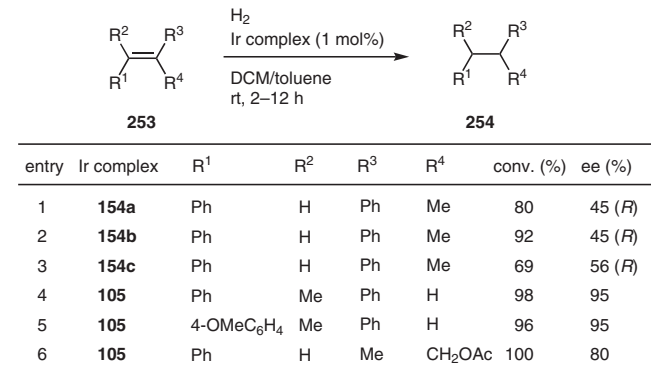

Scheme 61 Iridium-catalyzed hydrogenation of alkenes ${ }^{88,93}$

\subsubsection{Asymmetric Hydrogenation of Ketones}

In 2005, Leitner and co-workers developed a highly enantioselective ruthenium-catalyzed hydrogenation of aromatic ketones with $\left(R_{\mathrm{a}}, S_{\mathrm{c}}\right)$-QUINAPHOS 99a in the presence of substituted and non-substituted diamines as co-catalysts. The hydrogenation results obtained are summarized in Scheme 62.

Later, in 2010, Baratta et al. employed ruthenium metal complexes (MC) 255a-d and osmium complexes $\mathbf{7 7}$ and $\mathbf{7 8}$ in the presence of $t$-BuOK, to catalyze chemoselective asymmetric hydrogenation ( $5 \mathrm{~atm} \mathrm{H}_{2}$ ) of aromatic and aliphatic ketones to give the desired chiral alcohols in high conversions and good selectivities (Scheme 62). 

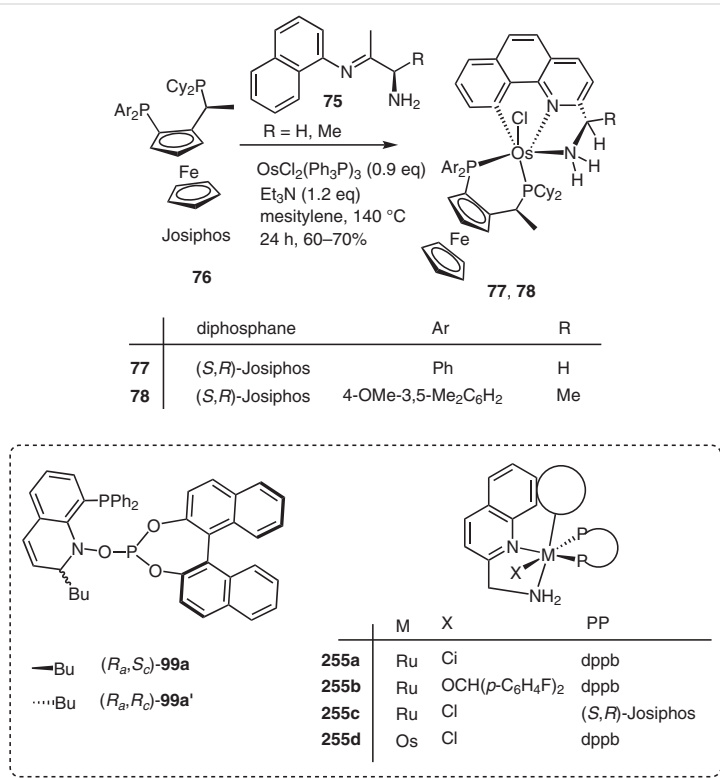

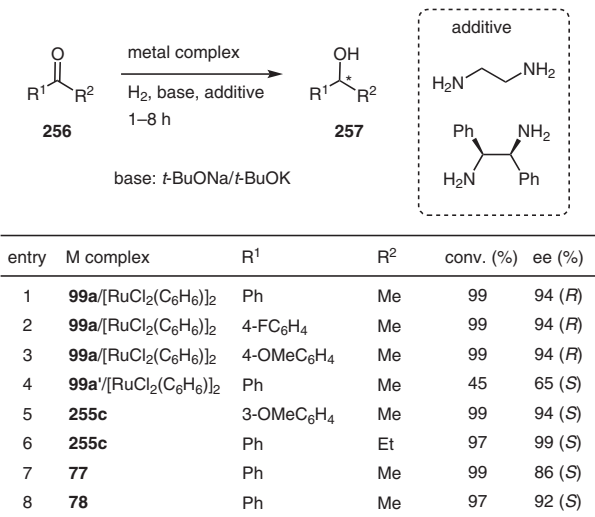

Scheme 62 Asymmetric hydrogenation of ketones in the presence of chiral metal complexes ${ }^{83 a, b, 108}$

\subsubsection{Asymmetric Hydrogenation of Imines}

In 2010, Ding and co-workers reported a chiral ligand bearing a spiro-scaffold-based Ir-complex 147 and successfully applied it in the enantioselective hydrogenation of aryl-imine 258, furnishing the corresponding chiral amine with enantioselectivities up to $58 \%$ ee (Scheme 63).

\subsection{Asymmetric Hydroformylation of Styrene}

Rh-catalyzed asymmetric hydroformylation of styrene 260 in the presence of $\mathrm{P}, \mathrm{N}$-chiral quinoline ligand 99a was reported by Leitner and co-workers in 2007. The P,N-chiral $\mathrm{Rh}$ complexes were applied to asymmetric hydroformylations of mono-substituted alkenes to give the correspond-

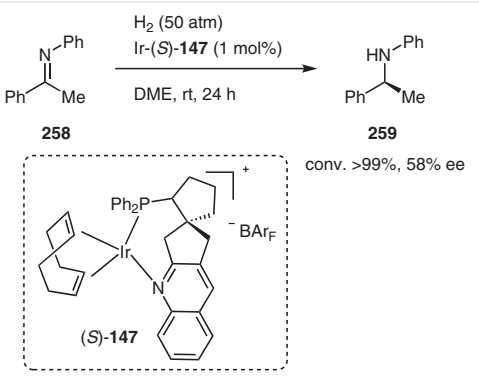

Scheme 63 Asymmetric hydrogenation of imines catalyzed by chiral iridium complex ${ }^{92 b}$

ing product $\mathbf{2 6 1}$ with up to $74 \%$ enantiomeric excess, with a linear aldehyde by-product $\mathbf{2 6 2}$ also being observed (Scheme 64).

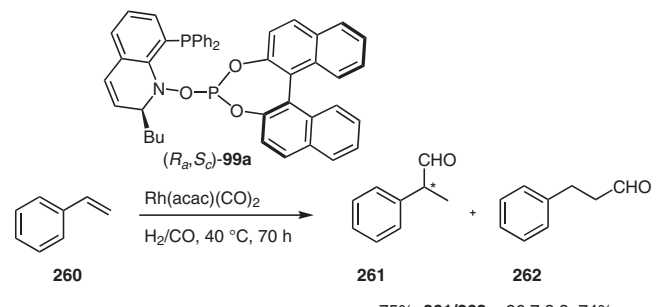

Scheme 64 Rhodium-catalyzed hydroformylation of styrene $e^{87 b}$

\subsection{Asymmetric Dialkoxylation of 2-Propenylphe- nols}

Sigman and co-workers (2007) successfully developed a direct $\mathrm{O}_{2}$-coupled $\mathrm{Pd}(\mathrm{II})$-catalyzed enantioselective dialkoxylation of 2-alkenylphenols by using quinoline oxazoline ligands 21a-d (Scheme 65). Pd(II)-catalyzed enantioselective dialkoxylation of 2-alkenylphenols 263, at room temperature for 24-72 h furnished the desired phenol 264 with enantioselectivities up to $92 \%$ ee.

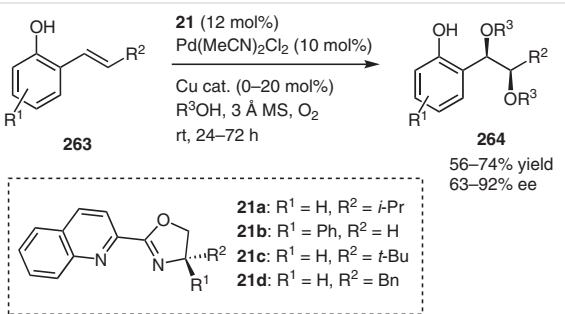

Scheme 65 Scope of Pd(II)-catalyzed enantioselective dialkoxylation ${ }^{109}$

\subsection{Asymmetric Cascade Cyclizations}

In 2009 Yang and co-workers reported the structurally tunable and an air-stable oxazoline 21c-Pd catalyst system for the highly enantioselective oxidative cascade intramolecular cyclization reaction of a variety of substituted bis- 
olefins 265, with excellent enantioselectivities (up to $98 \%$ ee), good yields and high diastereoselectivities $(\mathrm{dr}>24: 1)$ (Scheme 66).

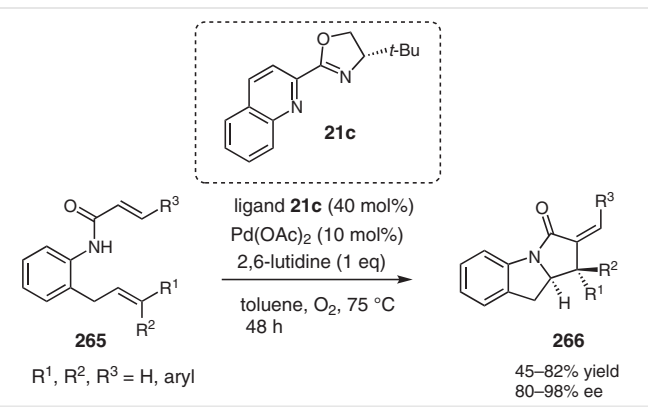

Scheme $66 \mathrm{Pd}(\mathrm{II})$-catalyzed enantioselective cascade cyclization ${ }^{72 c}$

\subsection{Asymmetric Allylic Alkylations}

Several chiral phosphine-quinoline ligand analogues were found to be good candidates for Pd-catalyzed asymmetric allylic alkylation reactions, as reported by Jiang et al. in 2008. Catalytic allylic alkylation has been demonstrated to be a powerful tool for stereoselective carbon-carbon bond-formation reactions in the presence of palladiumnitrogen ligand systems. Among many quinoline-based ligands designed for this chiral reaction, chiral bi- and tridentate type $\mathrm{P}, \mathrm{N}-$ ligands have played a significant role owing to their electronic and steric parameters. The reactions were carried out using 1.0-6.4 mol\% Pd catalyst and 2.5$12.8 \mathrm{~mol} \%$ chiral quinoline ligand. The results from a range of ligands are summarized in Scheme 67. Other protocols have been successfully examined for allylic alkylation reactions using various phosphine-quinoline based chiral ligands as outlined in Scheme 67. ${ }^{72,74,77,84,89,90,94,110}$

Trost and co-workers (2002) investigated Mo-catalyzed enantioselective allylic alkylations with sodium dimethyl malonate in the presence of diamide or amine type ligands 62. Allylic alkylation of ester $\mathbf{2 7 0}$ with sodium dimethyl malonate 271 furnished the corresponding chiral product 272 in low yields but high enantioselectivities (up to 98\%) (Scheme 68).

\subsection{Asymmetric Alkylation of $\beta$-Keto Esters}

Buono and co-workers studied the use of the palladium catalyst QUIPHOS 81a as a chiral ligand in the enantioselective alkylation of $\beta$-keto esters $\mathbf{2 7 3}$ with allyl substrate $\mathbf{2 7 4}$, leading to chiral products with high enantioselectivity (up to $95 \%$ ee) depending on the nature of the substrates and specific reaction conditions. In particular, solvents such as THF led to poor enantioselectivity (5-30\% ee); whereas the alkylation reaction performed with a five-membered-ring keto ester in DCM at $-10{ }^{\circ} \mathrm{C}$ gave the desired product 275 in $75 \%$ yield and high enantiomeric excess (95\% ee) (Scheme $69)$.
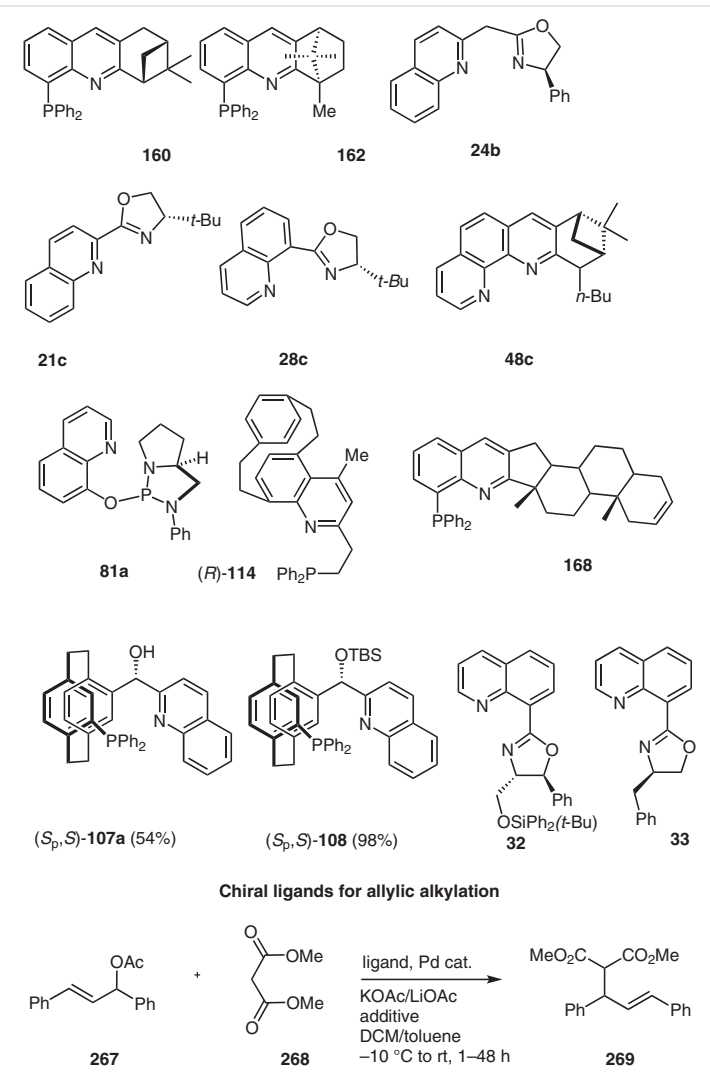

\begin{tabular}{|c|c|c|c|c|c|}
\hline \multicolumn{2}{|c|}{ entry ligand (mol\%) } & \multirow{2}{*}{$\begin{array}{l}\text { Pd cat. }(\mathrm{mol} \%) \\
{\left[\mathrm{Pd}\left(\eta^{3}-\mathrm{C}_{3} \mathrm{H}_{5}\right) \mathrm{Cl}\right]_{2}(2.5)}\end{array}$} & \multirow{2}{*}{$\begin{array}{c}\text { additive } \\
\text { BSA }\end{array}$} & \multirow{2}{*}{$\begin{array}{l}\text { yield (\%) } \\
90-95\end{array}$} & \multirow{2}{*}{$\begin{array}{r}\text { ee }(\%) \\
69-74(S)\end{array}$} \\
\hline 1 & $160-168(10)$ & & & & \\
\hline 2 & $24 b(10)$ & {$\left[\mathrm{Pd}\left(\eta^{3}-\mathrm{C}_{3} \mathrm{H}_{5}\right) \mathrm{Cl}\right]_{2}(2.5)$} & BSA & 88 & $78(S)$ \\
\hline 3 & $21 c(10)$ & {$\left[\mathrm{Pd}\left(\eta^{3}-\mathrm{C}_{3} \mathrm{H}_{5}\right) \mathrm{Cl}\right]_{2}(2.5)$} & BSA & 93 & $92(S)$ \\
\hline 4 & $28 c(10)$ & {$\left[\mathrm{Pd}\left(\eta^{3}-\mathrm{C}_{3} \mathrm{H}_{5}\right) \mathrm{Cl}\right]_{2}(2.5)$} & BSA & 94 & $77(S)$ \\
\hline 5 & $48 c(10)$ & {$\left[\mathrm{Pd}\left(\eta^{3}-\mathrm{C}_{3} \mathrm{H}_{5}\right) \mathrm{Cl}\right]_{2}(2.5)$} & BSA & 93 & $84(R)$ \\
\hline 6 & $81 a$ & {$\left[\mathrm{Pd}(\pi \text {-allyl) } \mathrm{Cl}]_{2}\right.$} & - & $>90$ & 85 \\
\hline 7 & $114(5)$ & {$[\mathrm{Pd}(\pi-\mathrm{ally} \mid) \mathrm{Cl}]_{2}(1.0)$} & BSA & $60^{\mathrm{a}}$ & $58(S)$ \\
\hline 8 & $107(6.0)$ & {$\left[\mathrm{Pd}\left(\eta^{3}-\mathrm{C}_{3} \mathrm{H}_{5}\right) \mathrm{Cl}\right]_{2}(2.0)$} & BSA & 98 & $96(R)$ \\
\hline 9 & $108(6.0)$ & {$\left[\mathrm{Pd}\left(\eta^{3}-\mathrm{C}_{3} \mathrm{H}_{5}\right) \mathrm{Cl}\right]_{2}(2.0)$} & BSA & 67 & $96(R)$ \\
\hline 10 & $32(2.5)$ & {$\left[\mathrm{Pd}\left(\eta^{3}-\mathrm{C}_{3} \mathrm{H}_{5}\right) \mathrm{Cl}\right]_{2}(1.0)$} & BSA & 85 & $65(S)$ \\
\hline 11 & $33(2.5)$ & {$\left[\mathrm{Pd}\left(\eta^{3}-\mathrm{C}_{3} \mathrm{H}_{5}\right) \mathrm{Cl}\right]_{2}(1.0)$} & BSA & 98 & $69(R)$ \\
\hline
\end{tabular}

Scheme 67 Asymmetric allylic alkylation (AAA) of 1,3-diphenylprop2-enyl acetate with dimethyl malonate ${ }^{72 a, b, 74,77 a, b, 84 c, 89,90,94 a, b, 110}$

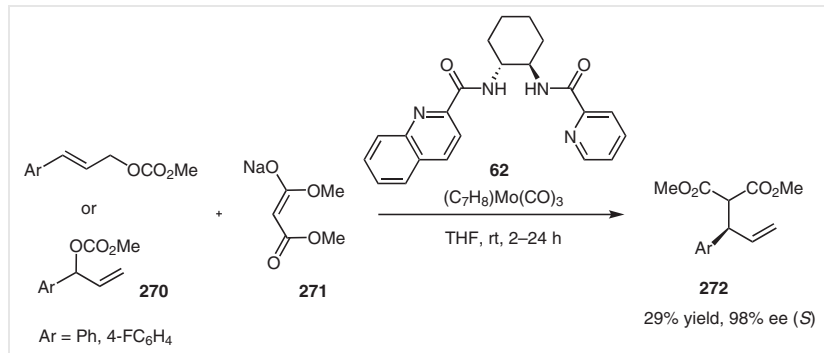

Scheme 68 Mo-catalyzed asymmetric allylic alkylation with sodium dimethyl malonate ${ }^{111}$ 


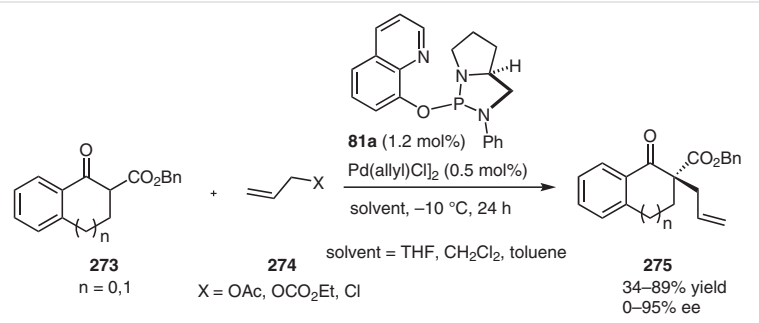

Scheme 69 Palladium-catalyzed asymmetric allylic alkylation of $\beta$-keto esters $^{112}$

\subsection{Asymmetric C-H Bond Arylation Reactions}

In 2019, Yu, Bertrand and co-workers studied the C-C bond coupling reaction reactivity and selectivity of quinoline-based amine ligands $\mathbf{2 7 8}$ in palladium-catalyzed $\beta-\mathrm{C}\left(\mathrm{sp}^{3}\right)-\mathrm{H}$ bond asymmetric arylation reactions. They disclosed the ligand synthesis, isolation, and detailed characterization of APAPy (acetyl-protected aminoethylpyridine) and APAQ (acetyl-protected aminoalkyl quinoline) ligands (Scheme 70). ${ }^{113}$
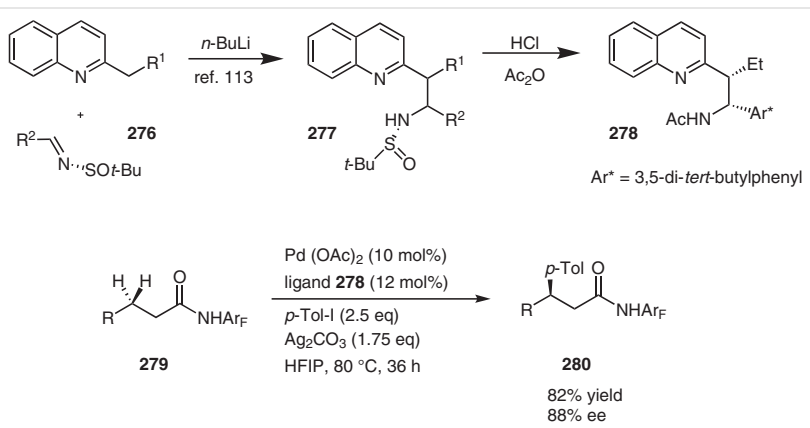

Scheme 70 Palladium catalyzed $\mathrm{C}-\mathrm{H}$ bond arylation reactions ${ }^{113}$

\subsection{Intramolecular Aerobic Oxidative Amination of Alkenes}

Stahl and co-workers (2011) described the enantioselective aerobic oxidative amination of cyclic alkenes $\mathbf{2 8 1}$ in the presence of chiral quinoline-oxazoline ligand 21d. The intramolecular addition of alkenes with a protected amine in the presence of Pd catalyst $5 \mathrm{~mol} \%$ and chiral quinolineoxazoline ligands 21d (7.5 mol\%) gave the corresponding product in low yield but with up to $66 \%$ enantiomeric excess (Scheme 71).

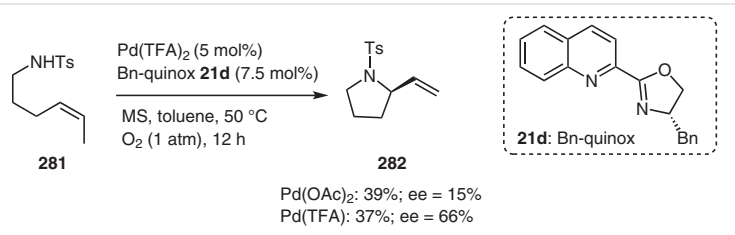

Scheme 71 Enantioselective oxidative amination employing a quinoline oxazoline ligand ${ }^{114}$

\subsection{Asymmetric Oxidative Hydroboration of Alkenes}

Brown and co-workers systematically studied the asymmetric hydroboration/oxidation of vinyl-arenes 284 at ambient temperature in the presence of rhodium complexes of 1,1'-(2-diarylphosphino-1-naphthyl)isoquinolines. Vinylarene substrates $\mathbf{2 8 4}$ bearing electron-withdrawing or -donating groups on the aryl ring led to the desired alcohol 286 with enantioselectivities up to $94 \%$ ee in the most favorable cases. The enantioselectivity of this specific conversion is moderately sensitive to the structure of the phosphorus type ligand, with the difurylphosphino ligand 121b furnishing excellent results using an electron-deficient styrene 284. Diphenylphosphino-ligand 121a showed the best results using an electron-donating alkene substrate (Scheme 72). ${ }^{75,91}$

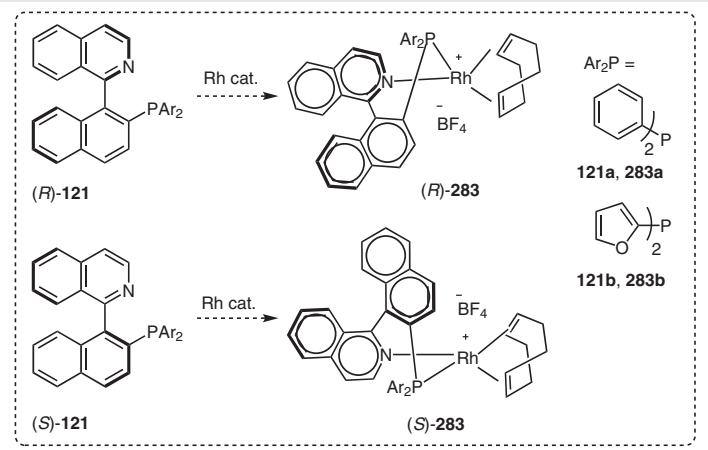

\begin{tabular}{|c|c|c|c|c|}
\hline & & \multicolumn{2}{|c|}{$\begin{array}{l}\text { 1) [LRh cat.], toluene } \\
\mathrm{rt}, 1-2 \mathrm{~h}\end{array}$} & \\
\hline & 285 & 2) $\begin{array}{r}\mathrm{H}_{2} \mathrm{O}_{2}, \\
\mathrm{rt}, 1-2\end{array}$ & & 286 \\
\hline entry & $\mathrm{Ar}$ & ligand & yield (\%) & ee $(\%)$ \\
\hline 1 & 2,4- $-\mathrm{Me}_{2} \mathrm{C}_{6} \mathrm{H}_{3}$ & $(R)-121 \mathrm{a}$ & 78 & $94(R)$ \\
\hline 2 & 4-OEtC ${ }_{6} \mathrm{H}_{4}$ & $(R)-121 \mathrm{a}$ & 82 & $94(R)$ \\
\hline 3 & 3- $-\mathrm{ClC}_{6} \mathrm{H}_{4}$ & $(S)-121 \mathbf{b}$ & 75 & $89(S)$ \\
\hline 4 & 2-naphthyl & $(R)-121 \mathrm{a}$ & 80 & $89(R)$ \\
\hline 5 & $\mathrm{Ph}$ & (S)-121a & 71 & $91(S)$ \\
\hline 6 & $\mathrm{Ph}$ & $(R)-121 \mathrm{a}$ & 70 & $67(R)$ \\
\hline
\end{tabular}

Scheme 72 Enantioselective oxidative hydroboration of electron-rich and electron-deficient vinylarenes ${ }^{75 b, 91 b, c}$ 


\section{Conclusions}

This review compiles the advancement in the synthesis of chiral ligands containing quinoline motifs and their catalytic asymmetric reactions. The potential of chiral quinolines and their metal complexes has been demonstrated in numerous catalytic asymmetric reactions such as the addition of dialkylzinc to aldehydes and enones, addition of Grignard reagents to enones, Michael addition reactions, Friedel-Crafts alkylations, aldol lactonizations, allylic oxidations, Diels-Alder reactions, Pudovik reactions, pinacol coupling reactions, Strecker reactions, cyclopropanations of olefins, Heck reactions, hydrogenation reactions, cascade cyclizations, allylic alkylations, $\mathrm{C}-\mathrm{H}$ bond arylation reactions, and oxidative hydroborations. We believe that this review will direct researchers to develop further methodologies for the synthesis of chiral quinoline-based ligands and to explore their new applications in asymmetric catalysis.

\section{Conflict of Interest}

The authors declare no conflict of interest.

\section{Funding Information}

The authors would like to thank the Department of Science and Technology, India (DST-Ref.No.: SB/FT/CS-117/2014), the Science and Engineering Research Board (SERB-Ref.No.: EEQ/2018/000574)Ramanujan Fellowship, and the National Institute of Technology Puducherry, Karaikal, India for providing financial support. This research was supported by the SERB-DST Grant No. RJF/2020/000038. V.D. gratefully acknowledges the Ramanujan Fellowship. The author RD acknowledge ICT-IOC, Bhubaneswar for providing necessary support. Rambabu Dandela thanks DST-SERB for Ramanujan fellowship (SB/S2/RJN-075/2016), Core research grant (CRG/2018/000782) and ICT-IOC start-up grant. K.B.D. would like to acknowledge the support from the Management, Principal-Dr. S. Sumaya and the Director-Research \& Industry-Institute Relations-Dr. M.S. Irfan Ahmed, Thassim Beevi Abdul Kader College for Women, Kilakarai, Ramanathapuram, Tamil Nadu, India.

\section{References}

(1) Collin, G.; Höke, H. Ullmann's Encyclopedia of Industrial Chemistry 2012, 31, 1 .

(2) Mehdi, F.-M. Mini-Rev. Org. Chem. 2017, 14, 187.

(3) Bose, D. S.; Idrees, M.; Jakka, N. M.; Rao, J. V. J. Comb. Chem. 2010, 12, 100.

(4) Marco-Contelles, J.; Pérez-Mayoral, E.; Samadi, A.; Carreiras, M. d. C.; Soriano, E. Chem. Rev. 2009, 109, 2652.

(5) Bharate, J. B.; Bharate, S. B.; Vishwakarma, R. A. ACS Comb. Sci. 2014, 16, 624 .

(6) Mastalir, M.; Glatz, M.; Pittenauer, E.; Allmaier, G.; Kirchner, K. J. Am. Chem. Soc. 2016, 138, 15543.

(7) Beesu, M.; Mehta, G. J. Org. Chem. 2019, 84, 8731.

(8) Willumstad, T. P.; Boudreau, P. D.; Danheiser, R. L. J. Org. Chem. 2015, 80, 11794.

(9) Weyesa, A.; Mulugeta, E. RSC Adv. 2020, 10, 20784.
(10) Marella, A.; Tanwar, O. P.; Saha, R.; Ali, M. R.; Srivastava, S.; Akhter, M.; Shaquiquzzaman, M.; Alam, M. M. Saudi Pharm. J. 2013, $21,1$.

(11) Meyet, C. E.; Larsen, C. H. J. Org. Chem. 2014, 79, 9835.

(12) Cretton, S.; Breant, L.; Pourrez, L.; Ambuehl, C.; Marcourt, L.; Ebrahimi, S. N.; Hamburger, M.; Perozzo, R.; Karimou, S.; Kaiser, M.; Cuendet, M.; Christen, P. J. Nat. Prod. 2014, 77, 2304.

(13) Boyd, D. R.; Sharma, N. D.; Loke, P. L.; Malone, J. F.; McRoberts, W. C.; Hamilton, J. T. Org. Biomol. Chem. 2007, 5, 2983.

(14) Campbell, S. F.; Hardstone, J. D.; Palmer, M. J. J. Med. Chem. 1988, $31,1031$.

(15) Markees, D. G.; Dewey, V. C.; Kidder, G. W. J. Med. Chem. 1970, $13,324$.

(16) Mabire, D.; Coupa, S.; Adelinet, C.; Poncelet, A.; Simonnet, Y.; Venet, M.; Wouters, R.; Lesage, A. S. J.; Beijsterveldt, L. V.; Bischoff, F. J. Med. Chem. 2005, 48, 2134.

(17) Guandalini, L.; Norcini, M.; Varani, K.; Pistolozzi, M.; Gotti, C.; Bazzicalupi, C.; Martini, E.; Dei, S.; Manetti, D.; Scapecchi, S.; Teodori, E.; Bertucci, C.; Ghelardini, C.; Romanelli, M. N. J. Med. Chem. 2007, 50, 4993.

(18) Cui, J. J.; Shen, H.; Tran-Dubé, M.; Nambu, M.; McTigue, M.; Grodsky, N.; Ryan, K.; Yamazaki, S.; Aguirre, S.; Parker, M.; Li, Q.; Zou, H.; Christensen, J. J. Med. Chem. 2013, 56, 6651.

(19) León, B.; Fong, J. C. N.; Peach, K. C.; Wong, W. R.; Yildiz, F. H.; Linington, R. G. Org. Lett. 2013, 15, 1234.

(20) Michael, J. P. Nat. Prod. Rep. 2003, 20, 476.

(21) Zhang, X.; Jenekhe, S. A. Macromolecules 2000, 33, 2069.

(22) Jenekhe, S. A.; Lu, L.; Alam, M. M. Macromolecules 2001, 34, 7315.

(23) Zhang, Z.; Shi, Y.; Pan, Y.; Cheng, X.; Zhang, L.; Chen, J.; Li, M.-J.; Yi, C. J. Mater. Chem. B 2014, 2, 5020.

(24) Pimpalshende, D. M.; Dhoble, S. J. Luminescence 2014, 29, 451.

(25) Biot, C.; Daher, W.; Chavain, N.; Fandeur, T.; Khalife, J.; Dive, D.; De Clercq, E. J. Med. Chem. 2006, 49, 2845.

(26) Manohar, S.; Rajesh, U. C.; Khan, S. I.; Tekwani, B. L.; Rawat, D. S. ACS Med. Chem. Lett. 2012, 3, 555.

(27) Ben-Zvi, I.; Kivity, S.; Langevitz, P.; Shoenfeld, Y. Clinic. Rev. Allerg. Immunol. 2012, 42, 145.

(28) Kumar, S.; Bawa, S.; Gupta, H. Mini-Rev. Med. Chem. 2009, 9, 1648.

(29) Muruganantham, N.; Sivakumar, R.; Anbalagan, N.; Gunasekaran, V.; Leonard, J. T. Biol. Pharm. Bull. 2004, 27, 1683.

(30) Luchi, R. J.; Conn, H. L.; Helwig, J. Am. J. Cardiol. 1962, 10, 252.

(31) Nevin, R. L. Int. J. Parasitol. Drug. 2014, 4, 118.

(32) Dhayalan, V.; Gadekar, S. C.; Alassad, Z.; Milo, A. Nat. Chem. 2019, 11, 543 .

(33) Raed, A. A.; Dhayalan, V.; Barkai, S.; Milo, A. Chimia 2020, 74, 878.

(34) Dhayalan, V.; Mal, K.; Milo, A. Synthesis 2019, 51, 2845.

(35) Wang, D.; Weinstein, A. B.; White, P. B.; Stahl, S. S. Chem. Rev. 2018, 118, 2636.

(36) Flanigan, D. M.; Romanov-Michailidis, F.; White, N. A.; Rovis, T. Chem. Rev. 2015, 115, 9307.

(37) Kurono, N.; Ohkuma, T. ACS Catal. 2016, 6, 989.

(38) Tanriver, G.; Dedeoglu, B.; Catak, S.; Aviyente, V. Acc. Chem. Res. 2016, 49, 1250.

(39) Chen, D.-F.; Han, Z.-Y.; Zhou, X.-L.; Gong, L.-Z. Acc. Chem. Res. 2014, 47, 2365.

(40) (a) Carroll, M.; Guiry, P. J. Chem. Soc. Rev. 2014, 43, 819. (b) Rokade, B. V.; Barker, J.; Guiry, P. J. Chem. Soc. Rev. 2019, 48, 4766. 
(41) (a) Rokade, B.; Guiry, P. J. ACS Catal. 2018, 8, 624. (b) Connon, R.; Roche, B.; Rokade, B. J.; Guiry, P. J. Chem. Rev. 2021, 121, 6373. (c) List, B. Chem. Rev. 2007, 107, 5413. (d) Xie, Y.; List, B. Angew. Chem. Int. Ed. 2017, 56, 4936. (e) Liu, C.; Oblak, E. Z.; Vander Wal, M. N.; Dilger, A. K.; Almstead, D. K.; MacMillan, D. W. C. J. Am. Chem. Soc. 2016, 138, 2134. (f) Singh, G. S.; Yeboah, E. M. O. Rep. Org. Chem. 2016, 6, 47.

(42) Shen, Z.-L.; Dhayalan, V.; Benischke, A. D.; Greiner, R.; Karaghiosoff, K.; Mayer, P.; Knochel, P. Angew. Chem. Int. Ed. 2016, 55,5332 .

(43) Li, J.; Tan, E.; Keller, N.; Chen, Y.-H.; Zehetmaier, P. M.; Jakowetz, A. C.; Bein, T.; Knochel, P. J. Am. Chem. Soc. 2019, 141, 98.

(44) Chen, Q.; du Jourdin, X. M.; Knochel, P. J. Am. Chem. Soc. 2013, $135,4958$.

(45) Steib, A. K.; Fernandez, S.; Kuzmina, O. M.; Corpet, M.; Gosmini, C.; Knochel, P. Synlett 2015, 26, 1049.

(46) Kuzmina, O. M.; Steib, A. K.; Moyeux, A.; Cahiez, G.; Knochel, P. Synthesis 2015, 47, 1696.

(47) Bellan, A. B.; Kuzmina, O. M.; Vetsova, V. A.; Knochel, P. Synthesis 2017, 49, 188.

(48) Balkenhohl, M.; Valsamidou, V.; Knochel, P. Eur. J. Org. Chem. 2019, 5165.

(49) See ref. 41a.

(50) Cao, Y.; Zhang, S.; Antilla, J. C. ACS Catal. 2020, 10, 10914.

(51) Mihorianu, M.; Leonzio, M.; Monari, M.; Ravotto, L.; Ceroni, P.; Bettinelli, M.; Piccinelli, F. ChemistrySelect 2016, 1, 1996.

(52) Shao, Y.-D.; Dong, M.-M.; Wang, Y.-A.; Cheng, P.-M.; Wang, T.; Cheng, D.-J. Org. Lett. 2019, 21, 4831.

(53) Batista, V. F.; Pinto, D. C. G. A.; Silva, A. M. S. ACS Sustainable Chem. Eng. 2016, 4, 4064.

(54) Wang, Q.; Zhang, W.-W.; Song, H.; Wang, J.; Zheng, C.; Gu, Q.; You, S.-L. J. Am. Chem. Soc. 2020, 142, 15678.

(55) Fernandes, A.; Laye, C.; Pramanik, S.; Palmeira, D.; Pekel, Ö. Ö.; Massip, S.; Schmidtmann, M.; Müller, T.; Robert, F.; Landais, Y. J. Am. Chem. Soc. 2020, 142, 564.

(56) Ingalls, E. L.; Holtzen, G. A.; Kaminsky, W.; Michael, F. E. J. Organomet. Chem. 2017, 832, 9.

(57) Wang, J.; Chen, M. W.; Ji, Y.; Hu, S. B.; Zhou, Y. G. J. Am. Chem. Soc. 2016, 138, 10413.

(58) Parvez, M. M.; Haraguchi, N.; Itsuno, S. Macromolecules 2014, 47, 1922.

(59) Tong, M.; Wang, S.; Zhuang, J.; Qin, C.; Li, H.; Wang, W. Org. Lett. 2018, 20, 1195.

(60) Shao, Y.; Han, D.; Dong, M.-M.; Yang, X.; Cheng, D.-J. Org. Chem. Front. 2021, 8, 605.

(61) Zheng, L.; Zhan, Y.; Yu, C.; Huang, F.; Wang, Y.; Jiang, H. Org. Lett. 2017, 19, 1482.

(62) Wang, S.-J.; Wang, Z.; Tang, Y.; Chen, J.; Zhou, L. Org. Lett. 2020, $22,8894$.

(63) Chen, J.; Fu, Y.; Yu, Y.; Wang, J.-R.; Guo, Y.-W.; Li, H.; Wang, W. Org. Lett. 2020, 22, 6061.

(64) Friestad, G. K.; Ji, A.; Baltrusaitis, J.; Korapala, C. S.; Qin, J. J. Org. Chem. 2012, 77, 3159.

(65) Thaler, T.; Geittner, F.; Knochel, P. Synlett 2007, 2655.

(66) Felluga, F.; Baratta, W.; Fanfoni, L.; Pitacco, G.; Rigo, P.; Benedetti, F. J. Org. Chem. 2009, 74, 3547.

(67) Hu, X.; Dawson, S. J.; Nagaoka, Y.; Tanatani, A.; Huc, I. J. Org. Chem. 2016, 81, 1137.

(68) (a) Kawamura, K.; Fukuzawa, H.; Hayashi, M. Org. Lett. 2008, 10, 3509. (b) Kawamura, K.; Fukuzawa, H.; Hayashi, M. Bull. Chem. Soc. Jpn. 2011, 84, 640.
(69) (a) Ramesh, N.; Prakash, C.; Sureshbabu, R.; Dhayalan, V.; Mohanakrishnan, A. K. Tetrahedron 2008, 64, 2071. (b) Tan, Q.; Hayashi, M. Adv. Synth. Catal. 2008, 350, 2639. (c) Dhayalan, V.; Murakami, R.; Hayashi, M. Asian J. Chem. 2013, 25, 7505. (d) Hayashi, M.; Yamada, K.; Nakayama, S.; Hayashi, H.; Yamazaki, S. Green Chem. 2000, 6, 257. (e) Sano, Y.; Tanaka, T.; Hayashi, M. Chem. Lett. 2007, 12, 1414.

(70) (a) Suga, H.; Kakehi, A.; Mitsuda, M. Bull. Chem. Soc. Jpn. 2004, 77, 561. (b) Suga, H.; Nakajima, T.; Itoh, K.; Kakehi, A. Org. Lett. 2005, 7, 1431. (c) Shi, J. W.; Zhao, M. X.; Lei, Z. Y.; Shi, M. J. Org. Chem. 2008, 73, 305. (d) Suga, H.; Funyu, A.; Kakehi, A. Org. Lett. 2007, 11, 97.

(71) Retmane, A.; Gmouh, S.; Runghen, M.; Valnot, J. Y.; Maddaluno, J.; Toupet, L.; Oulyadi, H.; Eddine, J. J. Tetrahedron: Asymmetry 2008, 19, 1523.

(72) (a) Chelucci, G.; Pinna, G. A.; Saba, A.; Valenti, R. Tetrahedron: Asymmetry 2000, 11, 4027. (b) Chelucci, G.; Gladiali, S.; Saba, A. Tetrahedron: Asymmetry 1999, 10, 1393. (c) He, W.; Yip, K. T.; Zhu, N. Y.; Yang, D. Org. Lett. 2009, 11, 5626.

(73) Fraile, J. M.; García, J. I.; Osés, G. J.; Mayoral, J. A.; Roldán, M. Organometallics 2008, 27, 2246.

(74) Canal, J. M.; Gómez, M.; Jiménez, F.; Rocamora, M.; Muller, G.; Duńach, E.; Franco, D.; Jiménez, A.; Cano, F. H. Organometallics 2000, 19, 966.

(75) (a) Park, S. W.; Son, J. H.; Kim, S. G.; Ahn, K. H. Tetrahedron: Asymmetry 1999, 10, 1903. (b) Chelucci, G.; Orrù, G.; Pinna, G. A. Tetrahedron 2003, 59, 9471. (c) Clark, C. R.; Hay, R. W. J. Chem. Soc., Dalton Trans. 1974, 2148.

(76) Bolm, C.; Verrucci, M.; Simic, O.; Cozzi, P. G.; Raabe, G.; Okamura, H. Chem. Commun. 2003, 2826.

(77) (a) Chelucci, G.; Saba, A.; Sanna, G.; Soccolini, F. Tetrahedron: Asymmetry 2000, 11, 3427. (b) Chelucci, G.; Saba, A. Tetrahedron: Asymmetry 1998, 9, 2575.

(78) (a) Takenaka, N.; Xia, G.; Yamamoto, H. J. Am. Chem. Soc. 2004, 126, 13198. (b) Abell, J. P.; Yamamoto, H. J. Am. Chem. Soc. 2008, $130,10521$.

(79) See ref. 17.

(80) Kwong, H. L.; Yeung, H. L.; Yeung, C. T.; Lee, W. S.; Lee, C. S.; Wong, W. L. Coord. Chem. Rev. 2007, 251, 2188.

(81) (a) Qi, G.; Judeh, Z. M. A. Tetrahedron: Asymmetry 2010, 21, 429. (b) Qi, G.; Ji, Y. Q.; Judeh, Z. M. A. Tetrahedron 2010, 66, 4195.

(82) Martinez, R.; Zoli, L.; Cozzi, P. G.; Ramon, D. J.; Yus, M. Tetrahedron: Asymmetry 2008, 19, 2600.

(83) (a) Baratta, W.; Fanfoni, L.; Magnolia, S.; Siega, K.; Rigo, P. Eur. J. Inorg. Chem. 2010, 1419. (b) Baratta, W.; Ballico, M.; Baldino, S.; Chelucci, G.; Herdtweck, E.; Siega, K.; Magnolia, S.; Rigo, P. Chem. Eur. J. 2008, 14, 9148. (c) See ref. 66.

(84) (a) Delapierre, G.; Brunel, J. M.; Constantieux, T.; Buono, G. Tetrahedron: Asymmetry 2001, 12, 1345. (b) Delapierre, G.; Constantieux, T.; Brunel, J. M.; Buono, G. Eur. J. Org. Chem. 2000, 2507. (c) Brunel, J. M.; Constantieux, T.; Buono, G. J. Org. Chem. 1999, 64, 8940.

(85) Delapierre, G.; Achard, M.; Buono, G. Tetrahedron Lett. 2002, 43, 4025.

(86) Franciò, G.; Drommi, D.; Graiff, C.; Faraone, F.; Tiripicchio, A. Inorg. Chim. Acta 2002, 338, 59.

(87) (a) Franciò, G.; Arena, C. G.; Faraone, F.; Graiff, C.; Lanfranchi, M.; Tiripicchio, A. Eur. J. Inorg. Chem. 1999, 1219. (b) Franciò, G.; Faraone, F.; Leitner, W. Angew. Chem. Int. Ed. 2000, 39, 1428.

(88) (a) Dhayalan, V.; Sämann, C.; Knochel, P. Chem. Commun. 2015, 51, 3239. (b) Dhayalan, V.; Alcañiz, F. R.; Werner, V.; Karaghiosoff, K.; Knochel, P. Synthesis 2015, 47, 3972. (c) Sämann, C.; Dhayalan, V.; Schreiner, P. R.; Knochel, P. Org. 
Lett. 2014, 16, 2418. (d) Schlücker, T.; Dhayalan, V.; Langhals, H.; Sämann, C.; Knochel, P. Asian J. Org. Chem. 2015, 4, 763. (e) Bunlaksananusorn, T.; Knochel, P. J. Org. Chem. 2004, 69, 4595. (f) See ref. 65.

(89) Jiang, B.; Lei, Y.; Zhao, X. L. J. Org. Chem. 2008, 73, 7833.

(90) Ruzziconi, R.; Santi, C.; Spizzichino, S. Tetrahedron: Asymmetry 2007, 18, 1742.

(91) (a) Alcock, N. W.; Brown, J. M.; Htimes, D. I. Tetrahedron: Asymmetry 1993, 4, 743. (b) Valk, J. M.; Whitlock, G. A.; Layzell, T. P.; Brown, J. M. Tetrahedron: Asymmetry 1995, 6, 2593. (c) Doucet, H.; Fernandez, E.; Layzell, T. P.; Brown, J. M. Chem. Eur. J. 1999, 5, 1320. (d) See ref. 75b.

(92) (a) Markò, I. E.; Vanherck, J. C.; Ates, A.; Tinant, B.; Declercq, J. P. Tetrahedron Lett. 2003, 44, 3333. (b) Han, Z.; Wang, Z.; Zhang, X.; Ding, K. Tetrahedron: Asymmetry 2010, 21, 1529.

(93) Drury, W. J. III.; Zimmermann, N.; Keenan, M.; Hayashi, M.; Kaiser, S.; Goddard, R.; Pfaltz, A. Angew. Chem. Int. Ed. 2004, 43, 70.

(94) (a) Chelucci, G.; Orrù, G. Tetrahedron Lett. 2005, 46, 3493. (b) Chelucci, G.; Baldino, S. Tetrahedron: Asymmetry 2006, 17, 1529.

(95) Allen, D. G.; Mclaughlin, G. M.; Robertson, G. B.; Steffen, W. L.; Salem, G.; Wild, S. B. Inorg. Chem. 1982, 21, 1007.

(96) (a) Thummel, R. P.; Lefoulon, F. J. Org. Chem. 1985, 50, 666. (b) Chelucci, G.; Thummel, R. P. Chem. Rev. 2002, 102, 3129. (c) Saito, M.; Nakajima, M.; Hashimoto, S. Chem. Commun. 2000, 1851. (d) Saito, M.; Nakajima, M.; Hashimoto, S. Tetrahedron 2000, 56, 9589. (e) Chelucci, G.; Murineddu, G.; Pinna, G. A. Tetrahedron: Asymmetry 2004, 15, 1373. (f) Malkov, A. V.; Dufková, L.; Farrugia, L.; Koćovský, P. Angew. Chem. Int. Ed. 2003, 42, 3674. (g) Jiao, Z.; Feng, X.; Liu, B.; Chen, F.; Zhang, G.; Jiang, Y. Eur. J. Org. Chem. 2003, 3818. (h) Liu, B.; Feng, X.; Chen, F.; Zhang, G.; Cui, X.; Jiang, Y. Synlett 2001, 1551.

(97) (a) Dhayalan, V.; Knochel, P. Synthesis 2015, 47, 3246. (b) Meyers, A. I.; Wettlaufer, D. G. J. Am. Chem. Soc. 1984, 106, 1135. (c) Meyers, A. I. J. Org. Chem. 2005, 70, 6137.

(98) Arena, C. G.; Calabro, G.; Francio, G.; Faraone, F. Tetrahedron: Asymmetry 2000, 11, 2387.

(99) Qi, G.; Judeh, Z. M. A. Synth. Commun. 2012, 42, 1585.

(100) Nakajima, M.; Yamamoto, S.; Yamaguchi, Y.; Nakamura, S.; Hashimoto, S. Tetrahedron 2003, 59, 7307.

(101) Jia, Y. X.; Zhu, S. F.; Yang, Y.; Zhou, Q. L. J. Org. Chem. 2006, 71, 75.
(102) (a) Tan, Q.; Hayashi, M. Org. Lett. 2009, 11, 3314. (b) Tanaka, T.; Tan, Q.; Iwanaga, K.; Hayashi, M. Carbohydr. Res. 2011, 346, 340.

(103) (a) Malkov, A. V.; Orsini, M.; Pernazza, D.; Muir, K. W.; Langer, V.; Meghani, P.; Koćovský, P. Org. Lett. 2002, 4, 1047. (b) Wrzeszcz, Z.; Siedlecka, R. Catalysts 2021, 4, 444.

(104) Brunel, J. M.; Campo, B. D.; Buono, G. Tetrahedron Lett. 1998, 39 , 9663.

(105) Suga, H.; Furihata, Y.; Sakamoto, A.; Itoh, K.; Yukihisa, Okumura. Y.; Tsuchida, T.; Kakehi, A.; Baba, T. J. Org. Chem. 2011, 76, 7377.

(106) Fraile, J. M.; García, J. I.; José, A.; Mayoral, J. A.; Roldán, M. Org. Lett. 2007, 9, 731.

(107) See ref. 73.

(108) Burk, S.; Franciò, G.; Leitner, W. Chem. Commun. 2005, 3460.

(109) (a) Zhang, Y.; Sigman, M. S. J. Am. Chem. Soc. 2007, 129, 3076. (b) Jensen, K. H.; Webb, J. D.; Sigman, M. S. J. Am. Chem. Soc. 2010, 132, 17471.

(110) (a) Zhang, R.; Xie, B.; Chen, G.-S.; Qiu, L.; Chen, Y.-X. Tetrahedron Lett. 2016, 57, 845. (b) Pamìes, O.; Margalef, J.; Cañellas, S.; James, J.; Judge, J.; Guiry, P. J.; Moberg, C.; Bäckvall, J.-E.; Pfaltz, A.; Pericas, M. A.; Diéguez, M. Chem. Rev. 2021, 121, 4373. (c) Chelucci, G.; Medici, S.; Saba, A. Tetrahedron: Asymmetry 1999, 10, 543. (d) Fekner, T.; Bunz, H. M.; Guiry, P. J. Eur. J. Org. Chem. 2008, 5055. (e) Drommi, D.; Saporita, M.; Bruno, G.; Faraone, F.; Scafato, P.; Rosini, C. Dalton Trans. 2007, 1509. (f) Sureshbabu, R.; Saravanan, V.; Dhayalan, V.; Mohanakrishnan, A. K. Eur. J. Org. Chem. 2011, 922. (g) Dhayalan, V.; Clement, J. A.; Jagan, R.; Mohanakrishnan, A. K. Eur. J. Org. Chem. 2009, 531. (h) Mohanakrishnan, A. K.; Dhayalan, V.; Clement, J. A.; Sureshbabu, R. B. R.; Kumar, N. S. Tetrahedron Lett. 2008, 49, 5850.

(111)Trost, B. M.; Dogra, K.; Hachiya, I.; Emura, T.; Hughes, D. L.; Krska, S.; Reamer, R. A.; Palucki, M.; Yasuda, N.; Reider, P. J. Angew. Chem. Int. Ed. 2002, 41, 1929.

(112) Brunel, J. M.; Tenaglia, A.; Buono, G. Tetrahedron: Asymmetry 2000, 11, 3585.

(113) (a) Romero, E. A.; Chen, G.; Gembicky, M.; Jazzar, R.; Yu, J.-Q.; Bertrand, G. J. Am. Chem. Soc. 2019, 141, 16726. (b) Andrä, M. S.; Schifferer, L.; Pollok, C. H.; Merten, C.; Gooßen, L. J.; Yu, J.-Q. Chem. Eur. J. 2019, 25, 8503.

(114) McDonald, R. I.; White, P. B.; Weinstein, A. B.; Tam, C. P.; Stahl, S. S. Org. Lett. 2011, 13, 2830. 